\title{
D-instantons, string field theory and two dimensional string theory
}

\author{
Ashoke Sen \\ Harish-Chandra Research Institute, HBNI, \\ Chhatnag Road, Jhusi, Allahabad 211019, India \\ E-mail: sen@hri.res.in
}

Abstract: In [4] Balthazar, Rodriguez and Yin (BRY) computed the one instanton contribution to the two point scattering amplitude in two dimensional string theory to first subleading order in the string coupling. Their analysis left undetermined two constants due to divergences in the integration over world-sheet variables, but they were fixed by numerically comparing the result with that of the dual matrix model. If we consider n-point scattering amplitudes to the same order, there are actually four undetermined constants in the world-sheet approach. We show that using string field theory we can get finite unambiguous values of all of these constants, and we explicitly compute three of these four constants. Two of the three constants determined this way agree with the numerical result of BRY within the accuracy of numerical analysis, but the third constant seems to differ by $1 / 2$. We also discuss a shortcut to determining the fourth constant if we assume the equality of the quantum corrected D-instanton action and the action of the matrix model instanton. This also agrees with the numerical result of BRY.

KeYwords: D-branes, String Field Theory

ArXiv EPrint: 2012.11624 


\section{Contents}

1 Introduction and summary 1

2 Background $\quad 8$

2.1 String field theory 8

$\begin{array}{ll}2.2 & \text { Dealing with the open string tachyons } \\ & 12\end{array}$

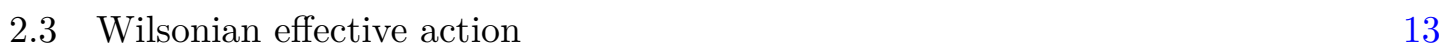

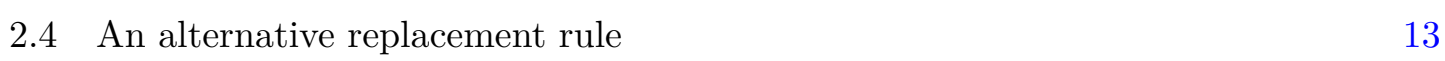

$\begin{array}{ll}2.5 & \text { Dealing with the zero mode fields } \\ \end{array}$

3 Approximations $\quad 16$

4 Interaction vertices and covering of the moduli space 20

$\begin{array}{lll}4.1 & \mathrm{C}-\mathrm{O} \text { amplitude on the disk } & 20\end{array}$

4.2 O-O-O amplitude on the disk 21

4.3 C-O-O amplitude on the disk 21

4.4 C-C amplitude on the disk 23

4.5 C-C-O amplitude on the disk 24

$\begin{array}{lll}4.6 & \text { O amplitude on the annulus } & 29\end{array}$

$\begin{array}{lll}4.7 & \mathrm{C} \text { amplitude on the annulus } & 31\end{array}$

5 Disk two point function in two dimensional string theory $\quad 37$

6 Annulus one point function in two dimensional string theory 38

$\begin{array}{lll}6.1 & \text { World-sheet contribution } & 39\end{array}$

$6.2 \psi^{1}$ exchange contribution 42

6.3 C-C-O disk amplitude, zero mode field redefinition and the Jacobian 44

6.4 C-O-O-O disk amplitude, ghost field redefinition and the Jacobian 48

$\begin{array}{ll}6.5 \text { Total contribution } & 51\end{array}$

7 Strategy for computing the correction to the D-instanton action 51

8 Some open problems $\quad 53$

A Disk two point function in general coordinate system $\quad 55$

B $\psi^{1}$ exchange contribution to the annulus one point function $\quad 57$

$\begin{array}{ll}\text { C Computation related to collective mode redefinition } & 60\end{array}$

$\begin{array}{ll}\text { D Computation related to gauge parameter redefinition } & 64\end{array}$ 
E Closed string tachyon one point function on the annulus with a more $\begin{array}{ll}\text { general C-O-O vertex } & 73\end{array}$

$\begin{array}{lll}\text { E.1 Modification of the annulus one point function } & 75\end{array}$

$\begin{array}{lll}\text { E.2 Modification of the C-C-O disk amplitude and its consequences } & 75\end{array}$

E.3 Modification of the $\psi^{1}$ exchange contribution 75

E.4 Modification of the C-O-O-O amplitude and its consequences 76

\section{Introduction and summary}

D-instantons represent saddle points of the path integral in second quantized string theory and give non-perturbative contribution to the string amplitudes. The usual worldsheet approach to computing these corrections suffers from infra-red divergences from the boundaries of the moduli spaces of Riemann surfaces. Furthermore, unlike the perturbative amplitudes in string theory, these infra-red divergences cannot be removed by analytic continuation in the external momenta, since the open strings living on the D-instanton carry strictly zero momentum. Even if the divergences cancel at the end [1-3], the fact that they are present in the intermediate stages of the analysis and need to be tamed by suitable regulator, makes the final result ambiguous.

A concrete example of such ambiguities arose in a recent analysis of two dimensional string theory [4]. The authors of [4] computed the D-instanton contribution to the closed string scattering amplitude, but could determine the final result only up to two undetermined constants. These constants were then determined by comparison with the results in the dual matrix model [5-9]. This comparison was carried out numerically, leading to the best fit values -1.399 and .496 for these constants. ${ }^{1}$

String field theory is well poised to address the issues related to infra-red divergences arising in string theory [11-13]. It does so by drawing insights from quantum field theory. Indeed, in an earlier analysis [14], string field theory was used to determine the first of these constants unambiguously, leading to the value $-\ln 4$, which is within $1 \%$ of the numerical result -1.399 . In this paper, we shall use the systematic procedure for carrying out perturbation theory in the presence of D-instantons, as developed in [15], to rederive this result in a more general set-up. We shall also see that to the order to which the analysis of $[4,14]$ was carried out, there are actually four independent constants that are apparently divergent / ambiguous in the world-sheet description, but can be fixed in string field theory. The second constant $\simeq .496$ that appeared in the analysis of [4] is a particular linear combination of these four constants. We determine three of these four constants explicitly using string field theory. Determination of the fourth constant is possible within string field theory, but involves computations more elaborate than what will be performed in this paper. All of these constants can also be determined independently by (numerically)

\footnotetext{
${ }^{1}$ Ref. [10] extended this analysis to multi-instanton contribution. However since the analysis was done only for the leading order terms in the expansion in powers of the string coupling around the multi-instanton solution, the ambiguities of the type discussed in this paper did not arise in [10].
} 
comparing the string theory results with the known results for higher point amplitudes in the matrix model. This has now been done [16]. We find that two of the three constants, determined from string field theory, agree with the results of the matrix model. However, the third constant differs by an additive constant close to $1 / 2$.

Since most of the paper will be technical in nature, we shall now give an overview of the main results. We begin by describing our convention. A naive amplitude with Dinstanton boundary condition does not satisfy energy conservation, since time translation invariance is broken by the Dirichlet boundary condition on the time coordinate. As will be discussed later, energy conservation is eventually restored after integration over the collective modes of the D-instanton, but it will be convenient for us to work with the amplitude before integration over the collective coordinates. Furthermore, we focus on amplitudes on connected world-sheets, since the contribution from world-sheets with more than one connected component can be found by taking the product of the contributions from the connected components. We shall refer to these as the building blocks from which the final amplitude will be constructed. Furthermore, every D-instanton amplitude is eventually multiplied by a factor of $\mathcal{N} e^{-1 / g_{s}}$ where $g_{s}$ is the string coupling constant and $\mathcal{N}$ is a numerical constant. We shall drop this factor from the building blocks. The final amplitude is to be constructed after taking sums of products of appropriate building blocks and then including an overall factor of $\mathcal{N} e^{-1 / g_{s}}$ and the energy conserving delta function. Finally, we shall use a convention in which all energies are counted as outgoing, i.e. an ingoing state of energy $\omega$ will be described as a state of energy $-\omega$. In this convention the final energy conserving delta function that comes from integration over the collective coordinate takes the form $2 \pi \delta\left(\omega_{\text {tot }}\right)$ where $\omega_{\text {tot }}$ is the total energy carried by all the external closed string states in an amplitude.

Ref. [4] computed three building blocks in two dimensional string theory. One is the one point disk amplitude of a closed string tachyon of energy $\omega$. This is given by:

$$
2 \sinh (\pi|\omega|) .
$$

The powers of $g_{s}$ in this amplitude are explained as follows. The disk gives a factor of $1 / g_{s}$, and the closed string vertex operator is normalized so that it carries a factor of $g_{s}$. Therefore the powers of $g_{s}$ cancel from this amplitude. The second building block computed in [4] is the two point function of a pair of closed string tachyons of energy $\omega_{1}$ and $\omega_{2}$ on the disk. This takes the form:

$$
4 g_{s} \sinh \left(\pi\left|\omega_{1}\right|\right) \sinh \left(\pi\left|\omega_{2}\right|\right) f\left(\omega_{1}, \omega_{2}\right),
$$

where

$$
\begin{aligned}
f\left(\omega_{1}, \omega_{2}\right)= & 2^{-1 / 4} \pi^{1 / 2} 2^{\left(\omega_{1}^{2}+\omega_{2}^{2}\right) / 2} \frac{1}{\sinh \left(\pi\left|\omega_{1}\right|\right) \sinh \left(\pi\left|\omega_{2}\right|\right)} \int_{0}^{1} d y \\
& \times y^{\omega_{2}^{2} / 2}(1-y)^{1-\omega_{1} \omega_{2}}(1+y)^{1+\omega_{1} \omega_{2}}\left\langle V_{\left|\omega_{1}\right| / 2}(i) V_{\left|\omega_{2}\right| / 2}(i y)\right\rangle_{\mathrm{UHP}} .
\end{aligned}
$$

Here $\left\langle V_{\left|\omega_{1}\right| / 2}(i) V_{\left|\omega_{2}\right| / 2}(i y)\right\rangle_{\text {UHP }}$ denotes the two point function of a pair of primaries in the $\mathrm{c}=25$ Liouville theory of momenta $\left|\omega_{1}\right| / 2$ and $\left|\omega_{2}\right| / 2$, inserted at $i$ and $i y$ respectively in 
the upper half plane. The third building block computed in [4] is the one point function of a closed string tachyon of energy $\omega$ on the annulus. This takes the form:

$$
2 g_{s} \sinh (\pi|\omega|) g(\omega)
$$

where

$$
g(\omega)=2 \pi^{2} \frac{1}{\sinh (\pi|\omega|)} \int_{0}^{\infty} d t \int_{0}^{1 / 4} d x \eta(i t)\left(\frac{2 \pi}{\theta_{1}^{\prime}(0 \mid i t)} \theta_{1}(2 x \mid i t)\right)^{\omega^{2} / 2}\left\langle V_{|\omega| / 2}(2 \pi x)\right\rangle_{A} .
$$

Here $\theta_{1}(z \mid \tau)$ is the odd Jacobi theta function and $\theta_{1}^{\prime}(z \mid \tau) \equiv \partial_{z} \theta_{1}(z \mid \tau) .\left\langle V_{|\omega| / 2}(2 \pi x)\right\rangle_{A}$ denotes the one point function of the Liouville primary of momentum $|\omega|$ on an annulus described by $0 \leq \operatorname{Re}(w) \leq \pi, w \equiv w+2 \pi i t$, with the vertex operator inserted at $\operatorname{Re}(w)=2 \pi x$. Explicit expressions for the correlation functions in the Liouville theory can be found in [4].

The integrals defining $f\left(\omega_{1}, \omega_{2}\right)$ and $g(\omega)$ are divergent from the $y \simeq 0, t \simeq \infty$ and $x \simeq 0$ regions. $^{2}$ We now divide each of these functions into a finite part and a divergent part:

$$
\begin{aligned}
f\left(\omega_{1}, \omega_{2}\right) & =f_{\text {finite }}\left(\omega_{1}, \omega_{2}\right)+f_{\operatorname{div}}\left(\omega_{1}, \omega_{2}\right), \\
g(\omega) & =g_{\text {finite }}(\omega)+g_{\text {div }}(\omega)
\end{aligned}
$$

where,

$$
\begin{aligned}
& f_{\text {finite }}\left(\omega_{1}, \omega_{2}\right)= 2^{-1 / 4} \pi^{1 / 2} 2^{\left(\omega_{1}^{2}+\omega_{2}^{2}\right) / 2} \frac{1}{\sinh \left(\pi\left|\omega_{1}\right|\right) \sinh \left(\pi\left|\omega_{2}\right|\right)} \int_{0}^{1} d y \\
& \times {\left[y^{\omega_{2}^{2} / 2}(1-y)^{1-\omega_{1} \omega_{2}}(1+y)^{1+\omega_{1} \omega_{2}}\left\langle V_{\left|\omega_{1}\right| / 2}(i) V_{\left|\omega_{2}\right| / 2}(i y)\right\rangle_{\mathrm{UHP}}\right.} \\
&\left.\quad-2^{-3 / 4} \pi^{-1 / 2} 2^{-\left(\omega_{1}^{2}+\omega_{2}^{2}\right) / 2} \sinh \left(\pi\left|\omega_{1}\right|\right) \sinh \left(\pi\left|\omega_{2}\right|\right) y^{-2}\left(1+2 \omega_{1} \omega_{2} y\right)\right] \\
& f_{\text {div }}\left(\omega_{1}, \omega_{2}\right)=\frac{1}{2} \int_{0}^{1} d y y^{-2}\left(1+2 \omega_{1} \omega_{2} y\right)
\end{aligned}
$$

and,

$$
\begin{aligned}
g_{\text {finite }}(\omega)= & 2 \pi^{2} \frac{1}{\sinh (\pi|\omega|)} \int_{0}^{\infty} d t \int_{0}^{1 / 4} d x\left[\eta(i t)\left(\frac{2 \pi}{\theta_{1}^{\prime}(0 \mid i t)} \theta_{1}(2 x \mid i t)\right)^{\omega^{2} / 2}\left\langle V_{|\omega| / 2}(2 \pi x)\right\rangle_{A}\right. \\
& \left.-\frac{1}{\pi} \sinh (\pi|\omega|)\left(\frac{e^{2 \pi t}-1}{\sin ^{2}(2 \pi x)}+2 \omega^{2}\right)\right] \\
g_{\text {div }}(\omega)= & 2 \pi \int_{0}^{\infty} d t \int_{0}^{1 / 4} d x\left(\frac{e^{2 \pi t}-1}{\sin ^{2}(2 \pi x)}+2 \omega^{2}\right) \\
= & \int_{0}^{1} d v \int_{0}^{1 / 4} d x\left\{\frac{v^{-2}-v^{-1}}{\sin ^{2}(2 \pi x)}+2 \omega^{2} v^{-1}\right\} .
\end{aligned}
$$

\footnotetext{
${ }^{2}$ There are also divergences from the $y \simeq 1$ and $t \simeq 0$ regions for real energies. These are associated with closed string degenerations and were avoided in [4] by working with imaginary external energies. In [17] we showed how to use string field theory to get finite results for these amplitudes for real energies.
} 
In the second step we have made a change of variable from $t$ to $v$ via $v=e^{-2 \pi t}$. and (1.11) suggests that we parametrize $f_{\text {div }}\left(\omega_{1}, \omega_{2}\right)$ and $g_{\text {div }}(\omega)$ as

$$
f_{\text {div }}\left(\omega_{1}, \omega_{2}\right)=A_{f}+B_{f} \omega_{1} \omega_{2}, \quad g_{\text {div }}(\omega)=A_{g}+B_{g} \omega^{2},
$$

where $A_{f}, B_{f}, A_{g}$ and $B_{g}$ are constants.

There is one more building block that was not analyzed in [4], but will be needed for our discussion below. This is the zero point function on surfaces of Euler number -1 , that includes a disk with two holes and a torus with one hole. Since there are no external momenta, we can parametrize it as $C g_{s}$ for some constant $C$.

Ref. [4] used these building blocks to construct a D-instanton induced two point closed string tachyon amplitude to order $g_{s}$, but we shall present the result in a more general form where we use the same building blocks to construct a general $(n+1)$-point amplitude of closed string tachyons to this order. The leading order contribution comes from the product of $n+1$ disk one point functions. Our interest will be in the subleading order $g_{s}$ contribution which comes from three sources: 1) the product of $(n-1)$ disk one point functions and one disk two point function, 2) the product of $n$ disk one point functions and one annulus one point function, and 3$)$ the product of $(n+1)$ disk one point functions and one zero point function on a surface of Euler number -1. A fourth contribution, given by the product of a sphere three point function and $(n-2)$ disk one point functions, has a separate energy conserving delta-function from the sphere three point function and does not contribute for generic energies of incoming and outgoing particles satisfying overall energy conservation. After restoring the factors of $\mathcal{N} e^{-1 / g_{s}}$ and the $2 \pi \delta\left(\sum_{i} \omega_{i}\right)$, the D-instanton induced $(n+1)$-point amplitude up to order $g_{s}$ takes the form:

$$
\begin{aligned}
A_{n+1}= & \mathcal{N} e^{-1 / g_{s}} 2 \pi \delta\left(\omega_{1}+\omega_{2}+\cdots \omega_{n}+\omega_{n+1}\right)\left[\prod_{i=1}^{n+1}\left\{2 \sinh \left(\pi\left|\omega_{i}\right|\right)\right\}\right] \\
& \times\left[1+g_{s} \sum_{\substack{i, j=1 \\
i<j}}^{n+1} f\left(\omega_{i}, \omega_{j}\right)+g_{s} \sum_{i=1}^{n+1} g\left(\omega_{i}\right)+C g_{s}\right] .
\end{aligned}
$$

Using (1.6), (1.7) and (1.12) we now get,

$$
\begin{aligned}
A_{n+1}= & 2^{n+1} \mathcal{N} e^{-1 / g_{s}} 2 \pi \delta\left(\omega_{1}+\omega_{2}+\cdots+\omega_{n+1}\right)\left\{\prod_{i=1}^{n+1} \sinh \left(\pi\left|\omega_{i}\right|\right)\right\} \\
& \times\left[1+g_{s} \sum_{\substack{i, j=1 \\
i<j}}^{n+1} f_{\text {finite }}\left(\omega_{i}, \omega_{j}\right)+g_{s} \sum_{i=1}^{n+1} g_{\text {finite }}\left(\omega_{i}\right)\right. \\
& \left.+\left\{\frac{1}{2} n(n+1) A_{f}+(n+1) A_{g}+C\right\} g_{s}+\left\{B_{g}-\frac{1}{2} B_{f}\right\} g_{s} \sum_{i=1}^{n+1} \omega_{i}^{2}\right],
\end{aligned}
$$

where in the last term we have used the energy conservation law to replace $\sum_{i<j} \omega_{i} \omega_{j}$ by $-\sum_{i} \omega_{i}^{2} / 2$. This shows that the collection of the amplitudes for different values of $n$ depends on the coefficients $A_{f}, A_{g}$ and $C$ individually, but only on the combination $2 B_{g}-B_{f}$. 
The matrix model result for the scattering amplitude of a closed string tachyon of energy $-\omega_{n+1}$ into $n$ closed string tachyons of energy $\omega_{1}, \cdots, \omega_{n}$ is known explicitly, and is given by $[4,17]$

$$
\begin{aligned}
2^{n+1} \mathcal{N} e^{-1 / g_{s}} 2 & \pi \delta\left(\omega_{1}+\omega_{2}+\cdots \omega_{n}+\omega_{n+1}\right)\left\{\prod_{i=1}^{n+1} \sinh \left(\pi\left|\omega_{i}\right|\right)\right\} \\
\times & {\left[1-i g_{s} \sum_{j=1}^{n} \omega_{j}\left(1-\sum_{i=1}^{n} \pi \omega_{i} \operatorname{coth}\left(\pi \omega_{i}\right)\right)\right], }
\end{aligned}
$$

with $\mathcal{N}=-1 /\left(8 \pi^{2}\right)$. Ref. [4] compared (1.14) and (1.15) for $n=1$ numerically and found that the results match well if we choose:

$$
A_{f}+2 A_{g}+C \simeq-.496, \quad 2 B_{g}-B_{f} \simeq-1.399 .
$$

We emphasize however that we can numerically compare (1.14) and (1.15) for different values of $n$ to extract the coefficients $A_{f}, A_{g}, C$ and $2 B_{g}-B_{f}$ separately. This has now been done, and the comparison yields [16]:

$$
A_{f} \simeq-0.50, \quad A_{g} \simeq 0.00, \quad C \simeq 0.00, \quad 2 B_{g}-B_{f} \simeq-1.40,
$$

with an expected error of order .01 in each term.

In this paper we use string field theory to show that,

$$
\begin{aligned}
g_{\text {div }}(\omega) & =-\frac{1}{2}+\frac{1}{2} \omega^{2} \ln \frac{\lambda^{2}}{4}, \\
f_{\text {div }}\left(\omega_{1}, \omega_{2}\right) & =-\frac{1}{2}\left(1-2 \omega_{1} \omega_{2} \ln \lambda^{2}\right),
\end{aligned}
$$

where $\lambda$ is an arbitrary parameter that enters the construction of string field theory. Different values of $\lambda$ give apparently different string field theories, but they can be shown to be related by field redefinition [18]. Comparison with (1.12) now gives:

$$
A_{f}=-\frac{1}{2}, \quad B_{f}=\ln \lambda^{2}, \quad A_{g}=-\frac{1}{2}, \quad B_{g}=\frac{1}{2} \ln \frac{\lambda^{2}}{4} .
$$

We see that $B_{f}$ and $B_{g}$ are individually ambiguous but the combination $2 B_{g}-B_{f}$ that enters the physical amplitudes is unambiguous and is given by:

$$
2 B_{g}-B_{f}=-\ln 4 \simeq-1.386 .
$$

Even though we have not computed $C$ explicitly, we have argued in section 7 that if we make the additional assumption that one can equate the renormalized D-instanton action with the renormalized action of the matrix model instanton, we get

$$
C=0 \text {. }
$$

The values of $A_{f}, 2 B_{g}-B_{f}$ and $C$ given in (1.19), (1.20) and (1.21) agree with the numerical values (1.17) determined by comparison with the matrix model within an error 
of about .01. However $A_{g}$ given in (1.19) seems to differ from the numerical value of $A_{g}$ given in (1.17) by an additive constant $-1 / 2$. While this discrepancy could be removed by rescaling the closed string tachyon field by $\left(1+\frac{1}{2} g_{s}\right)$, this option is not available since it would generate corrections of order $g_{s}$ in the perturbative amplitudes that are not present in the theory. The origin of this discrepancy is not clear to us. The most likely explanation is some missing contribution in the string field theory analysis, but we have not been able to identify such a contribution. Furthermore, as discussed in section 8 , our results pass various consistency tests. We discuss various other possible origins of this discrepancy in section 8 .

We shall now briefly mention the main subtleties that arise in our analysis.

1. The divergent parts of the amplitude are handled by interpreting these divergences in string field theory and following the standard procedure of quantum field theories to remove these divergences. This in particular requires us to not use the Schwinger parameter representation for the tachyon propagator that is commonly used in arriving at the world-sheet description of the amplitude. Instead we use the string field theory result for the tachyon propagator given by the inverse of the mass ${ }^{2}$ of the tachyon. This removes all power law divergences.

2. We also need to remove the zero mode fields propagating in the internal lines, and treat them as we would do in a quantum field theory. Once the contribution due to the zero mode fields is removed, all logarithmic divergences also disappear. The effect of integration over the zero mode fields will be discussed separately below.

3. In the D-instanton background the Siegel gauge, that is normally used in string field theory, breaks down and we need a different gauge fixing [15]. In this new gauge there is an additional field whose contribution to the amplitude is not captured in the usual world-sheet formulation. We need to explicitly include the contribution from this field propagating in the internal propagators in all the amplitudes.

4. The open string field theory has a zero mode field that represents the freedom of translating the D-instanton along the (euclidean) time direction. This can be related to the collective coordinate of the D-instanton by a field redefinition, after which the integration over the collective coordinate produces the usual energy conserving delta function. However, the original string field theory integration measure produces an additional Jacobian factor during this change of variable. This gives additional contributions to the amplitudes that need to be accounted for.

5. In the Siegel gauge the ghost sector contains a pair of zero mode fields, one of which is removed in the new gauge that we use. However the other one remains, and can be interpreted as the ghost field associated with a particular gauge symmetry of string field theory. Integration over this ghost field can be interpreted as division by the volume of the corresponding gauge group. Now this particular gauge symmetry is related, by a field dependent multiplicative factor, to the rigid $\mathrm{U}(1)$ gauge symmetry under which an open string stretched from the D-instanton under consideration to any other D-brane picks up a phase. Since the rigid U(1) gauge group has a constant 
volume, the effect of division by this volume can be absorbed into a redefinition of the overall normalization constant $\mathcal{N}$. However the field dependent multiplicative factor that takes us from the string field theory gauge transformation parameter to the rigid $\mathrm{U}(1)$ gauge transformation parameter gives rise to an additional Jacobian factor in the path integral measure. This also needs to be taken into account while computing amplitudes.

String field theory that we use has two ad hoc parameters $\lambda$ and $\widetilde{\lambda}$ and a function $f(\beta)$. The contribution to the coefficients (1.19) from the different effects mentioned above - the two jacobians, the effect of integration over the out of Siegel gauge modes, and the original contribution after removal of the zero mode contribution and the string field theory treatment of the tachyonic modes, all depend on these ad hoc parameters and the function $f(\beta)$ in a non-trivial way. An illustration of this can be found in (6.55), which lists contribution to $g_{\text {div }}(\omega)$ from various sources. However after summing over all the contributions, the dependence on these parameters and the function $f(\beta)$ disappears and we get the unambiguous results for the coefficients $A_{f}, A_{g}$ and $2 B_{g}-B_{f}$ as given in (1.19). This is consistent with the fact that different string field theories corresponding to different values of these ad hoc parameters and the function $f(\beta)$ are related by field redefinition [18].

The rest of the paper is organized as follows. In section 2 we review some aspects of string field theory and the main results of [15] that we shall be using to tame the infrared divergences in the computation of string amplitudes in a D-instanton background. One of the ingredients in this computation is the determination of the relationship between the standard coordinates of the moduli space of Riemann surfaces and the coordinates that arise from the string field theory description of the amplitudes. However it is often sufficient to determine approximate relations between these coordinates instead of the exact relations. In section 3 we estimate the errors that we make in using these approximations, and determine how much error we are allowed to make so as not to affect the final results. In section 4 we introduce the interaction vertices of string field theory by choosing appropriate local coordinates at the punctures of various Riemann surfaces. The latter are needed for describing off-shell amplitudes. Using these data, we find the (approximate) relations between the coordinates of the moduli space of Riemann surfaces and the parameters arising from string field theory description for various amplitudes. In section 5 we use these results to compute the D-instanton contribution to the two point disk amplitude of closed string tachyons in the two dimensional string theory, avoiding the infrared divergences by using the procedure described in section 2. This determines the function $f_{\text {div }}\left(\omega_{1}, \omega_{2}\right)$ leading to the result given in the second line of (1.18). In section 6 we use the results of section 4 to compute the D-instanton contribution to the one point function of closed string tachyon on the annulus. This determines $g_{\text {div }}(\omega)$, given in the first line of (1.18). In section 7 we discuss the steps needed to compute the two loop correction $C$ to the D-instanton action. However we stop short of actually doing this computation, which involves an open string two loop amplitude and a closed string one loop amplitude. Nevertheless we give an indirect determination of this constant $C$ by assuming the equality of the quantum corrected actions of the D-instanton and the matrix model instanton. We end in section 8 by listing some 
open questions. The appendices describe the details of some of the computations whose results were used in the text.

\section{Background}

In this section we shall give a brief introduction to some aspects of string field theory [1921] that we shall use, and also review the algorithm developed in [15] for carrying out systematic computation of closed string amplitudes in the presence of D-instantons. We shall focus on the contribution associated with a single D-instanton. Furthermore, we shall assume that the background does not have any other D-branes, so that all the boundaries of the world-sheet have D-instanton boundary conditions.

\subsection{String field theory}

The usual world-sheet description of string amplitudes involves integration over moduli spaces of Riemann surfaces with punctures, with the external closed string vertex operators inserted at the punctures in the bulk of the world-sheet and the external open string vertex operators inserted at the punctures on the boundary of the world-sheet. The integrand is computed from appropriate correlation functions of the vertex operators and worldsheet ghost fields on the Riemann surface. In the two dimensional string theory that we shall consider, the world-sheet theory consists of a free scalar field $X$ describing the time coordinate, a Liouville field theory with central charge 25, and the usual $b, c$ ghost system.

At a superficial level, string field theory may be regarded as a regular quantum field theory, whose perturbation expansion generates the same result at the one in the worldsheet approach. Each Feynman diagram of string field theory generates an integral similar to what we would get in the world-sheet approach, but the integration over the moduli runs over a subspace of the full moduli space. The sum over all Feynman diagrams gives back integration over the full moduli space. We shall see however, that this correspondence is only formal, - often the world-sheet expression is divergent while the corresponding string field theory expression yields finite result.

Although the relevant field theory that describes the interaction of closed strings and open strings on the D-instanton is the open-closed string field theory, since our goal will be to understand how to integrate over the open string fields, we shall regard the closed string fields as fixed background fields. In general there will be infinite number of closed string fields, but for most of our analysis in the two dimensional string theory, we shall only need the coupling of the on-shell closed string tachyon to the open string fields. So we shall only write down the coupling of the open string fields to the closed string tachyon field carrying energy $\omega$ and Liouville momentum $P$, which we shall denote by $\Phi_{C}(\omega, P)$. Despite its name, it actually describes a massless field in two dimensions. In the convention of [10], the on-shell condition takes the form $P=|\omega| / 2$.

If $H$ denotes the vector space of states of the open string, including matter and ghost excitations, then the off-shell open string field is taken to be an arbitrary element $|\Psi\rangle$ of 
$H$. This means that if $\left\{\left|\phi_{r}\right\rangle\right\}$ is the set of basis states in $H$, and if we expand $|\Psi\rangle \in H$ as

$$
|\Psi\rangle=\sum_{r} \chi^{r}\left|\phi_{r}\right\rangle
$$

then $\left\{\chi^{r}\right\}$ are the dynamical degrees of freedom on which the path integral is to be performed (after suitable gauge fixing). For string field theory on a $\mathrm{D} p$-brane the sum over $r$ will also include integration over $(p+1)$ continuous variables labelling the energy and momenta of the state, but on the D-instanton the open strings do not carry any continuous momenta and the sum over $r$ is a discrete sum. Therefore we can regard this as a field theory in zero space-time dimensions. The grassmann parity of $\chi^{r}$ is opposite to that of the state $\left|\phi_{r}\right\rangle$. Therefore the string field $\Psi$ is grassmann odd.

The string field theory action takes the form:

$$
S=\frac{1}{2}\left\langle\Psi\left|Q_{B}\right| \Psi\right\rangle+\cdots
$$

where $Q_{B}$ is the world-sheet BRST operator and $\cdots$ denotes interaction terms. Note that we have not explicitly written down the kinetic term for the closed string fields since they will be treated as background fields and the kinetic term will not be needed for our analysis. The interaction term takes the form

$$
\begin{aligned}
\sum_{m \geq 0} \sum_{n \geq 0} \frac{1}{m ! n !} \int & d \omega_{1} d P_{1} \cdots d \omega_{m} d P_{m} \Phi_{C}\left(\omega_{1}, P_{1}\right) \cdots \Phi_{C}\left(\omega_{m}, P_{m}\right) \\
& \times \chi^{r_{1}} \cdots \chi^{r_{n}} F_{r_{1} \cdots r_{n}}^{(m, n)}\left(\omega_{1}, P_{1}, \cdots, \omega_{m}, P_{m}\right)
\end{aligned}
$$

where the functions $F_{r_{1} \cdots r_{n}}^{(m, n)}\left(\omega_{1}, P_{1}, \cdots, \omega_{m}, P_{m}\right)$ are constructed from appropriate correlation functions of $m$ closed string tachyon vertex operators with energies $\omega_{1}, \cdots, \omega_{m}, n$ open string vertex operators $\phi_{r_{1}}, \cdots, \phi_{r_{n}}$ and appropriate set of $b$-ghost insertions, integrated over some appropriate subspaces of moduli space of Riemann surfaces with punctures. In general the functions $F_{r_{1} \cdots r_{n}}^{(m, n)}$ depend on the choice of local coordinate systems at the punctures, but since we shall need these functions only for on-shell closed string states, the dependence on the local coordinates at the punctures in the bulk of the world-sheet, where closed string vertex operators are inserted, drops out. For on-shell open string states the correlation function is also independent of the choice of local coordinate system at the punctures on the boundaries of the world-sheet, but this is not the case for off-shell open string states. Different choices of local coordinates lead to apparently different string field theories, but they can be shown to be related to each other by field redefinition [18].

The action (2.2) is to be regarded as the quantum master action in the BatalinVilkovisky formalism $[22,23]$. Let us assign ghost number to the states in $H$ by declaring $c, \bar{c}$ to have ghost number $1, b, \bar{b}$ to have ghost number -1 , matter fields to have ghost number 0 and the $\mathrm{SL}(2, R)$ invariant vacuum to have ghost number 0 . In the BV formalism we declare the open string fields multiplying states of ghost number $\leq 1$ as fields and the fields multiplying states of ghost number $\geq 2$ as antifields. To be more specific, let us introduce basis states $\left|\varphi_{r}\right\rangle$ in ghost number $\leq 1$ subspace and $\left|\varphi^{r}\right\rangle$ in the ghost number $\geq 2$ subspace such that

$$
\left\langle\varphi^{r} \mid \varphi_{s}\right\rangle=\delta_{s}^{r}=\left\langle\varphi_{s} \mid \varphi^{r}\right\rangle, \quad\left\langle\varphi^{r} \mid \varphi^{s}\right\rangle=0, \quad\left\langle\varphi_{r} \mid \varphi_{s}\right\rangle=0,
$$


and expand the string field as,

$$
|\Psi\rangle=\sum_{r}\left(\psi^{r}\left|\varphi_{r}\right\rangle+\psi_{r}\left|\varphi^{r}\right\rangle\right) .
$$

Then we declare $\psi^{r}$ as a field and $\psi_{r}$ as the conjugate anti-field (up to a sign).

String field theory constructed this way has gauge symmetries where the gauge transformation parameters are in also in one to one correspondence with states of the open string. Therefore we can represent a gauge transformation parameter as:

$$
|\Lambda\rangle=\sum_{r} \theta^{r}\left|\phi_{r}\right\rangle
$$

with $\theta^{r}$ 's being the parameters of infinitesimal gauge transformation. Even though $\theta^{r}$ and $\chi^{r}$ appear in similar expansions (2.1) and (2.6), their grassmann parities are opposite of each other. The significance of this will become clear later The gauge transformation of a field $\chi^{r}$ under a gauge transformation parameter $\theta^{s}$ is given (up to a sign) by the double derivative of the action with respect to $\chi_{r}^{*}$ and $\chi^{s}$, where $\chi_{r}^{*}$ is the anti-field conjugate to $\chi^{r}$, computed via (2.5) [24]. More general gauge transformations where the transformation parameters themselves are functions of fields have been discussed in [25], but we shall not discuss them here.

The actual path integral of string field theory is carried out over fields in a Lagrangian submanifold - this is the analog of gauge fixing in the Fadeev-Popov formalism. We shall not give a general definition of Lagrangian submanifolds, but it suffices to say that one set of choices correspond to setting, for each pair $\left(\psi^{r}, \psi_{r}\right)$, either $\psi^{r}$ to 0 or $\psi_{r}$ to 0 . This can be done independently for each $r$. The result of the path integral for physical quantities can be shown to be formally independent of the choice of the Lagrangian submanifold.

A particular choice of Lagrangian submanifold corresponds to setting all the anti-fields to 0 . Therefore $|\Psi\rangle$ has ghost number $\leq 1$. It then follows from ghost number conservation that the action depends on only the ghost number 1 sector fields. These are called classical fields since the physical open string states of the string belong to this sector. In this case the path integral decomposes into an integration over the classical fields on which the action depends and an integration over the fields of ghost number $\leq 0$ on which the action does not depend. Since the string fields $\chi^{r}$ have opposite grassmann parity compared to the corresponding gauge transformation parameters $\theta^{r}$, the integration over the string fields of ghost number $\leq 0$ can be interpreted as division by the volume of the gauge group generated by gauge transformation parameter $\Lambda$ carrying ghost number $\leq 0$. This connects the BV formalism to the usual path integral formulation of gauge theories, in which we start with the path integral over the classical fields weighted by the exponential of the classical action and divide this by the volume of the gauge group. While this is a sensible starting point when the gauge group has a finite volume, as in lattice gauge theories on a finite lattice and with a compact gauge group, this is usually not suitable for perturbation theory, since the kinetic operator of the classical action has flat directions associated with the gauge deformations. This makes the kinetic operator non-invertible, requiring us to gauge fix the theory. In the BV formalism this will correspond to choosing a different Lagrangian submanifold. 
The Lagrangian submanifold commonly used for carrying out perturbation theory is called the Siegel gauge. Here we restrict the path integral to a subspace of string fields of the form:

$$
b_{0}|\Psi\rangle=0 .
$$

More precisely this means the following. We can choose the basis states $\left|\varphi^{r}\right\rangle$ and $\left|\varphi_{r}\right\rangle$ in (2.5) in such a way that one of the following holds:

1. $b_{0}\left|\varphi^{r}\right\rangle=0, c_{0}\left|\varphi_{r}\right\rangle=0$. In this case we choose $\psi^{r}=0$.

2. $c_{0}\left|\varphi^{r}\right\rangle=0, b_{0}\left|\varphi_{r}\right\rangle=0$. In this case we choose $\psi_{r}=0$.

Since for each $r$ we set to zero either the field or the anti-field, this is clearly a Lagrangian submanifold. The corresponding $|\Psi\rangle$ given in (2.5) satisfies (2.7).

In the Siegel gauge, the kinetic operator $Q_{B}$ appearing in (2.2) reduces to $c_{0} L_{0}$ and the open string propagator is proportional to

$$
b_{0} L_{0}^{-1}=b_{0} \int_{0}^{1} d q q^{L_{0}-1}
$$

where $L_{n}$ 's denote the total Virasoro generators of the world-sheet conformal field theory of matter and ghosts. As we shall discuss later, equality of the two sides of (2.8) is somewhat formal and breaks down for states with $L_{0} \leq 0$, but let us proceed with this for now. In this case there is a simple algorithm to determine which region of the moduli space is covered by a given Feynman diagram. The local coordinate system at the punctures, needed for defining the interaction term of string field theory for off-shell external states, play an important role in this analysis. If we contract the external state $\chi^{r}$ from one interaction vertex with the external state $\chi^{s}$ from another (or the same) interaction vertex ${ }^{3}$ by a propagator, then geometrically this corresponds to sewing the Riemann surfaces associated with the two interaction vertices by identifying the corresponding local coordinates $w$ and $w^{\prime}$ via the relation

$$
w w^{\prime}=-q, \quad 0 \leq q \leq 1 .
$$

For this reason, we shall call $q$ the sewing parameter associated with the open string propagator. Following this procedure we can determine the regions in the moduli space of punctured Riemann surfaces covered by each Feynman diagram. This not only generates the family of Riemann surfaces corresponding to the Feynman diagram, but also generates the local coordinates at the punctures of these Riemann surfaces in terms of the local coordinates at the punctures of the Riemann surfaces associated with the interaction vertices. The interaction vertices are chosen such that the region corresponding to the sum of all Feynman diagrams covers the full moduli space. We shall discuss several examples of this in section 4. This condition is needed for string field theory to reproduce the usual world-sheet results for the amplitudes, but at a more fundamental level, this condition is also necessary for the action to satisfy the BV master equation.

\footnotetext{
${ }^{3}$ Throughout this paper an interaction vertex of string field theory will have the same meaning as in the Feynman diagrams of a quantum field theory, and should not be confused with vertex operators that refer to operators in the world-sheet theory.
} 
We shall use a convention in which the path integral measure is weighted by $e^{S}$. In this convention the contribution to an amplitude from a Feynman diagram containing a single interaction vertex is given directly by the corresponding function $F_{r_{1} \cdots r_{n}}^{(m, n)}\left(\omega_{1}, P_{1}, \cdots, \omega_{m}, P_{m}\right)$ without any additional sign or numerical factors. However we shall keep the overall normalization of $S$ arbitrary, by choosing an arbitrary normalization of the vacuum in the world-sheet theory (see (6.20)). This will have the advantage that we can use our result both in the Euclidean and in the Lorentzian theory (in which the standard measure is $e^{i S}$ ) by changing the overall normalization of $S$.

\subsection{Dealing with the open string tachyons}

We shall now review the divergences that arise in the world-sheet description of the amplitudes in the presence of D-instantons. They are of two kinds - those associated with the open string tachyons and those associated with the open string zero mode fields. In this subsection we shall describe some general aspects of D-instanton perturbation theory, and review the procedure discussed in [15] for dealing with the open string tachyons. The effect of open string zero modes will be discussed in section 2.5 .

1. In the presence of D-instantons we need to sum over contributions to string amplitudes from Riemann surfaces with boundaries, with the world-sheet fields satisfying boundary condition associated with the D-instantons at the boundaries. We need to include disconnected world-sheets in the computation, with the rule that each connected component must have either at least one external closed string insertions or at least one boundary with D-instanton boundary condition, and at least one of the connected components must have both, an external closed string insertion and a boundary.

2. The integrals over the moduli spaces of Riemann surfaces suffer from infra-red divergences from the boundaries of the moduli space. These divergences are most problematic for open string degenerations since the open strings living on the D-instanton do not carry any momentum and therefore the usual approach of dealing with the divergences by analytic continuation in the external momenta does not work. However, as we shall review below, string field theory can be used to deal with these divergences.

3. The infrared divergences associated with open string degeneration arise from the $q \simeq 0$ region on the right hand side of (2.8). As is obvious from (2.8), these divergences arise from $L_{0} \leq 0$ states. The divergences associated with $L_{0}<0$ states can be easily tackled by using the left hand side of (2.8) instead of the right hand side. This leads to the replacement rule:

$$
\int_{0}^{1} d q q^{\alpha-1} \rightarrow \frac{1}{\alpha} \quad \text { for } \alpha \neq 0 .
$$

For $\alpha>0$ this is an identity but for $\alpha<0$ this is a prescription that ensures that the world-sheet computations reproduce the string field theory results.

Since the $L_{0}<0$ states represent tachyonic modes, the reader may worry about treating them this way when they propagate in the loops. Indeed, this will not be a 
sensible way to treat tachyons in a quantum field theory. However, for D-instantons the tachyons do not carry any momentum and the path integral over the tachyons represent ordinary integrals, with the integrand having a local minimum in the direction of the tachyon field instead of a local maximum. This means that the steepest descent contour runs along the imaginary axis of the tachyon field. Once we take the integration contour over the tachyonic mode along this steepest descent contour, as is customary for evaluating contributions from saddle points of a path integral, there is no problem in carrying out systematic perturbation expansion to evaluate the contribution to the integral.

4. For treating the divergences associated with the $L_{0}=0$ states we need to introduce the notion of Wilsonian effective action that we shall discuss next.

\subsection{Wilsonian effective action}

In string field theory, it is possible to integrate out the set of fields corresponding to a given set of $L_{0}$ eigenvalues and write an effective action for the other fields. The resulting effective action shares all the properties of the original master action, both in the classical theory [26-29] and in the quantum theory [30]. We can use this to integrate out all the open string fields with $L_{0} \neq 0$, including the open string tachyon, leaving us with the master action of the $L_{0}=0$ fields only. We shall call this the Wilsonian effective action. The interaction vertices of this effective action are obtained by summing over all the Feynman diagrams of the original string field theory, with only the $L_{0}=0$ open string states (and closed string states) as external states, and by removing, from each internal open string propagator, the contribution from the $L_{0}=0$ states. From the world-sheet perspective, this corresponds to subtracting all contributions of the form $\int_{0}^{1} d q q^{-1}$ in the integrand. Combining this with (2.10) we arrive at the modified replacement rule:

$$
\int_{0}^{1} d q q^{\alpha-1} \rightarrow \frac{1}{\alpha}\left\{1-\delta_{\alpha, 0}\right\}
$$

The $\left\{1-\delta_{\alpha, 0}\right\}$ reflects that for $\alpha=0$, we need to remove the contribution from the region $0 \leq q \leq 1$ altogether. Since this removes all divergent terms, computation of the Wilsonian effective action does not suffer from any divergences.

\subsection{An alternative replacement rule}

We shall now describe an alternative to the replacement rule (2.11). We shall consider the cases $\alpha=0$ and $\alpha=-1$ only, since these are the relevant cases for our analysis. If we replace the lower limit of $q$ integration by some small number $\delta$, then (2.11) can also be stated as,

$$
\int_{\delta}^{1} d q q^{\alpha-1} \rightarrow \begin{cases}-1 & \text { for } \alpha=-1 \\ 0 & \text { for } \alpha=0\end{cases}
$$

On the other hand if we explicitly evaluate the left hand side, we get

$$
\int_{\delta}^{1} d q q^{\alpha-1}= \begin{cases}\delta^{-1}-1 & \text { for } \alpha=-1 \\ -\ln \delta & \text { for } \alpha=0 .\end{cases}
$$


Therefore the replacement rule simply corresponds to dropping the boundary terms proportional to $\delta^{-1}$ and $\ln \delta$.

This procedure is particularly useful if the integrand is a total derivative - which is indeed the case in many of the expressions that we shall encounter. In this case we can express this as an integral over the various boundary segments labeled by $q_{i}=\delta_{i}$ following the procedure described in [12], and then drop terms proportional to $\delta_{i}^{-1}$ and $\ln \delta_{i}$ in the final expression. Although we shall not describe this analysis in this paper, we have checked that following this procedure we get the same results as the ones reported here.

\subsection{Dealing with the zero mode fields}

At the level of the Wilsonian effective action, the open string fields associated with the $L_{0}=$ 0 states, which we shall collectively call the zero modes, remain unintegrated. However in computing physical amplitudes we have to eventually integrate over them. Doing this requires physical understanding of the origin of these fields.

1. First let us consider the zero modes arising from the pure ghost sector. We begin by writing down the full expansion of the string field in the $L_{0}=0$ sector, involving pure ghost excitations:

$$
\psi^{1} c_{0}|0\rangle+\psi^{2}|0\rangle+\psi_{1} c_{-1} c_{1}|0\rangle+\psi_{2} c_{-1} c_{0} c_{1}|0\rangle .
$$

The analog of the field $\psi^{1}$ in heterotic string theory has been identified in [31] with the zero mode of the Nakanishi-Lautrup field [32,33], while the fields $\psi^{2}$ and $\psi_{1}$ can be identified with the zero mode of the ghost and the anti-ghost fields associated with the U(1) gauge symmetry. Siegel gauge condition would set $\psi^{1}$ and $\psi_{2}$ to zero, but the resulting kinetic operator $c_{0} L_{0}$ will vanish while acting on the remaining $L_{0}=0$ states, leading to divergent propagator of the fields $\psi^{2}$ and $\psi_{1}$. For this reason, in the $L_{0}=0$ sector we choose a new gauge [15] in which we set all the anti-fields to zero, and express the string field involving pure ghost excitations as:

$$
\psi^{1} c_{0}|0\rangle+\psi^{2}|0\rangle+\cdots .
$$

2. We are supposed to integrate over the fields $\psi^{1}$ and $\psi^{2}$. First consider the effect of $\psi^{1}$. One can show that the field $\psi^{1}$ associated with the state $c_{0}|0\rangle$ has finite kinetic term and propagator, and can be treated perturbatively. This means however that besides the usual world-sheet contribution, with the replacement rule (2.11), we must also separately include the contribution from the propagator of this new field $\psi^{1}$ in every propagator of a Feynman diagram. A classical version of a similar result can be found in [31].

3. The field $\psi^{2}$ associated with the state $|0\rangle$ multiplies a state of ghost number zero. Using the same argument as for the full string field theory, one can show that it decouples from the action due to ghost number conservation. The interpretation of integration over $\psi^{2}$ follows from the same analysis that was used for the full string field theory - since the string field $\psi^{2}$ associated with the state $|0\rangle$ is grassmann odd, the 
integration over $\psi^{2}$ corresponds to dividing by the volume of gauge transformation in string field theory generated by $\theta|0\rangle$, where $\theta$ in the infinitesimal gauge transformation parameter. This gauge transformation, in turn, is related to the rigid $\mathrm{U}(1)$ gauge transformation with parameter $\tilde{\theta}$ after a suitable redefinition that relates $\theta$ to $\tilde{\theta}$. For example, under the rigid gauge transformation any open string that stretches from the original D-instanton to a second D-instanton picks up a phase $e^{i \widetilde{\theta}} \simeq(1+i \widetilde{\theta})$, but in general the transformation law under the gauge transformation generated by $\theta|0\rangle$ in open string field theory is more complicated. We can find the relation between $\theta$ and $\tilde{\theta}$ by comparing these transformation laws. If $\theta$ is related to $\tilde{\theta}$ as $\theta=J \tilde{\theta}$ where $J$ is some function of the fields, then $d \theta$ is given by $J d \tilde{\theta}$ and dividing by $\int d \theta$ is equivalent to dividing by $J \int d \tilde{\theta}$. Since $\int d \tilde{\theta}$ is the volume of the rigid $\mathrm{U}(1)$ gauge group, this contributes an overall constant to the path integral that can be absorbed into the normalization constant $\mathcal{N}$ in (1.13). Therefore the net effect of dividing by $\int d \theta$ is to include a factor of $J^{-1}=\exp [-\ln J]$ in the path integral. This can give additional contribution to the amplitudes that needs to be taken into account in our analysis.

4. Finally, let us turn to the zero modes coming from the matter sector in the ghost number 1 sector. Typically for single instanton, these arise from the collective excitations of the D-instanton that translate the D-instanton along transverse directions along which we have translational symmetry. For the two dimensional string theory that we shall analyze, there is only one such zero mode described by the vertex operator $c \partial X$ in the world-sheet theory. We shall denote this field by $\phi$. The integration over this mode is carried out at the end of the computation. In general, $\phi$ itself is not the collective coordinate, but it is expected to be related to the collective coordinate $\widetilde{\phi}$ describing time translation of the D-instanton by a field redefinition [13]. We can find this explicit field redefinition by noting that the dependence of the amplitude on the collective mode $\widetilde{\phi}$ must be proportional to $e^{-i \widetilde{\phi} \omega}$ where $\omega$ denotes the total outgoing energy of all the external closed string states in the amplitude. Therefore we first make the change of variable of integration in the path integral from $\phi$ to $\widetilde{\phi}$ so that the amplitude has the correct dependence on $\widetilde{\phi}$, and then perform the $\widetilde{\phi}$ integration to pick up the energy conserving delta function. Any Jacobian that arises from this change of variable must be taken into account in the computation of the amplitudes.

This discussion can be summarized by saying that after evaluating the amplitudes using the replacement rule (2.11) that renders the world-sheet integrals finite, we need to include the following extra contributions:

1. We need to take into account the contribution from the $\psi^{1}$ exchange in each internal propagator of the Feynman diagrams.

2. We need to take into account the additional contribution to the effective action from the Jacobian arising from change of variables from the open string matter sector zero modes to the collective coordinates.

3. We also need to take into account the additional contribution to the effective action from the Jacobian arising from change of variables from the open string gauge 
transformation parameter $\theta$ multiplying the vacuum state $|0\rangle$ to the rigid $\mathrm{U}(1)$ gauge transformation parameter. Both these Jacobians may give additional contribution to the amplitudes.

4. The amplitudes on the D-instanton do not conserve energy (and momentum). This is achieved at the very end when we integrate over the collective mode(s) $\widetilde{\phi}$ after changing variable from the open string matter sector zero mode(s) to the collective coordinate(s).

\section{Approximations}

In order to implement the procedure described in section 2, we need to know the precise relation between the integration parameters entering the expression for a world-sheet amplitude and the variables $q_{i}$ that are used to express the propagators via (2.8). We can then express the integral as integration over the variables $q_{i}$ 's near the $q_{i}=0$ region, expand the integrand in a power series expansion in the $q_{i}$ 's and make the replacement (2.11). Our goal in this section will be to analyze how much error we are allowed to make in identifying the variables $q_{i}$ without actually changing the value of the integral.

In order to convince the reader that this is not an empty exercise, we shall first demonstrate, with an example, that identifying the correct variables $q_{i}$ is important before applying the replacement rule (2.11). Let us consider an integral

$$
\int_{0}^{1} d q q^{-2} \rightarrow-1
$$

where we have used the replacement rule (2.11). Now suppose that instead of analyzing (3.1) directly, we first make a change of variable:

$$
q^{\prime}=\frac{q}{1-c q}
$$

with $c<1$, so that the change of variable is well defined in the range $0 \leq q \leq 1$. It is easy to check that $d q q^{-2}=d q^{\prime} q^{-2}$. Using this we can express (3.1) as

$$
\int_{0}^{1 /(1-c)} d q^{\prime} q^{\prime-2}=\int_{0}^{1} d q^{\prime} q^{\prime-2}+\int_{1}^{1 /(1-c)} d q^{\prime} q^{\prime-2} \rightarrow-1+\{1-(1-c)\}=-(1-c),
$$

where in the last but one step we have used the replacement rule (2.11) for the $q^{\prime}$ integral from 0 to 1 , and have explicitly evaluated the finite integral from 1 to $1 /(1-c)$. We now see that the final result in (3.3) differs from (3.1). This demonstrates that it is important to identify the variable $q$ correctly using string field theory before applying (2.11).

Before proceeding with the analysis, we shall make a change in the notation. For the type of string field theory we shall be considering, the integration parameters entering the expression for a world-sheet amplitude will have natural relation not with the $q_{i}$ 's but with $u_{i}=\epsilon_{i} q_{i}$ 's where $\epsilon_{i}$ are small parameters. In other words, the integrand, when expanded in powers of the $u_{i}$ 's, will have finite coefficients of expansion. In this case it is more convenient to trade in the variables $q_{i}$ 's for the $u_{i}$ 's, and express the replacement rule (2.11) by

$$
\int_{0}^{\epsilon_{i}} d u_{i} u_{i}^{\alpha-1} \rightarrow \frac{\epsilon_{i}^{\alpha}}{\alpha}\left\{1-\delta_{\alpha, 0}\right\}
$$


If we can determine the exact relation between the moduli parameters of the Riemann surface in terms of which we compute the integrand, and the $u_{i}$ 's, then we can carry out the procedure described above. However this is not strictly necessary - we shall show that even if we make some error in finding the relation between the moduli parameters and the $u_{i}$ 's, as long as the error goes to zero sufficiently fast in the $u_{i} \rightarrow 0$ limit, we can still get the result for the integral exactly. Our goal in this section will be to study how fast the error should vanish as $u_{i} \rightarrow 0$ so as to not make an error in the final value of the integral. This question is important since during our analysis we often will need to make approximations in relating the natural moduli space variables to the string field theory variables $q_{i}$, or equivalently, $u_{i}$.

We shall first consider the case where we have one integration variable $u$, and the integrand is $u^{-2}$. In this case the rule (3.4) can be stated more explicitly as:

$$
\mathcal{I} \equiv \int_{0}^{a} d u u^{-2}=\int_{0}^{\epsilon} d u u^{-2}+\int_{\epsilon}^{a} d u u^{-2} \rightarrow I \equiv-\frac{1}{\epsilon}+\int_{\epsilon}^{a} d u u^{-2},
$$

for any positive constant $a$. Note that $\mathcal{I}$ is a formal integral since the integral diverges from $u=0$, but $I$ is well defined. It can be easily verified that $I$ defined in (3.5) is independent of $\epsilon$. Now suppose that instead of using the variable $u$, we erroneously use the variable $v$, related to $u$ via

$$
u=f(v)=a_{0} v+a_{1} v^{2}+a_{2} v^{3}+\cdots,
$$

and use the replacement rule (3.4) with the variable $u$ replaced by $v$. Then the formal integral $\mathcal{I}$, when converted into an integration over the variable $v$, reduces to another formal integral:

$$
\mathcal{I}^{\prime}=\int_{0}^{b} d v f^{\prime}(v) f(v)^{-2}=\int_{0}^{\epsilon^{\prime}} d v f^{\prime}(v) f(v)^{-2}+\int_{\epsilon^{\prime}}^{b} d v f^{\prime}(v) f(v)^{-2}, \quad f(b)=a, \quad f\left(\epsilon^{\prime}\right)=\epsilon .
$$

Since

$$
f(v)^{-2} f^{\prime}(v)=a_{0}^{-1} v^{-2}+\mathcal{O}(1)
$$

we can express $\mathcal{I}^{\prime}$ as

$$
\mathcal{I}^{\prime}=\frac{1}{a_{0}} \int_{0}^{\epsilon^{\prime}} d v v^{-2}+\int_{0}^{\epsilon^{\prime}} d v\left\{f(v)^{-2} f^{\prime}(v)-a_{0}^{-1} v^{-2}\right\}+\int_{\epsilon^{\prime}}^{b} d v f(v)^{-2} f^{\prime}(v) .
$$

Note that only the first integral on the right hand side is divergent. If we now use the replacement rule (3.4) with $u$ replaced by $v$, we can replace the formal integral $\mathcal{I}^{\prime}$ by:

$$
\mathcal{I}^{\prime} \quad \rightarrow \quad I^{\prime} \equiv-\frac{1}{a_{0} \epsilon^{\prime}}+\int_{0}^{\epsilon^{\prime}} d v\left\{f(v)^{-2} f^{\prime}(v)-a_{0}^{-1} v^{-2}\right\}+\int_{\epsilon^{\prime}}^{b} d v f(v)^{-2} f^{\prime}(v) .
$$

It can be verified that the finite integral $I^{\prime}$ is independent of $\epsilon^{\prime}$. Using

$$
\int_{\epsilon^{\prime}}^{b} f(v)^{-2} f^{\prime}(v) d v=\int_{\epsilon}^{a} \frac{d u}{u^{2}}
$$

we get

$$
I^{\prime}=-\frac{1}{a_{0} \epsilon^{\prime}}+\int_{0}^{\epsilon^{\prime}} d v\left\{f(v)^{-1} f^{\prime}(v)-a_{0}^{-1} v^{-2}\right\}+\int_{\epsilon}^{a} \frac{d u}{u^{2}} .
$$


We can now use the $\epsilon$ independence of $I$ and $\epsilon^{\prime}$ independence of $I^{\prime}$ to evaluate both $I$ and $I^{\prime}$ in the limit $\epsilon, \epsilon^{\prime} \rightarrow 0$. Since (3.6) gives

$$
\epsilon=a_{0} \epsilon^{\prime}+a_{1} \epsilon^{\prime 2}+\cdots,
$$

we get, from (3.5), (3.12),

$$
I^{\prime}=I-\frac{a_{1}}{a_{0}^{2}}
$$

This shows that if instead of applying the replacement rule (3.4) on the original integral $\mathcal{I}$ expressed as integration over $u$, we first make a change of variables from $u$ to $v$ as given in (3.6), and then use analogous replacement rule on the integral, the result differs by $-a_{1} / a_{0}^{2}$. The example described at the beginning of this section is a special case of this general result.

Next we consider the case $\alpha=0$. In this case the replacement rule can be expressed as:

$$
\mathcal{J}=\int_{0}^{a} \frac{d u}{u} \quad \rightarrow \quad J \equiv \int_{\epsilon}^{a} \frac{d u}{u}
$$

This time $J$ does depend on the cut-off $\epsilon$ since the effect of this cut-off is to leave unintegrated the zero mode fields whose definition implicitly depends on the cut-off $\epsilon$. Under a change of variable of the form (3.6), the formal integral $\mathcal{J}$ may be expressed as,

$$
\begin{aligned}
\mathcal{J}^{\prime} & =\int_{0}^{b} f(v)^{-1} f^{\prime}(v) d v \\
& =\int_{0}^{\epsilon^{\prime}} v^{-1} d v+\int_{0}^{\epsilon^{\prime}}\left\{f(v)^{-1} f^{\prime}(v)-v^{-1}\right\} d v+\int_{\epsilon^{\prime}}^{b} f(v)^{-1} f^{\prime}(v) d v .
\end{aligned}
$$

Only the first term is divergent. Using the replacement rule (3.4) with $u$ replaced by $v$, we can now replace $\mathcal{J}^{\prime}$ by:

$$
\mathcal{J}^{\prime} \rightarrow J^{\prime} \equiv \int_{\epsilon}^{\epsilon^{\prime}} v^{-1} d v+\int_{0}^{\epsilon^{\prime}}\left\{f(v)^{-1} f^{\prime}(v)-v^{-1}\right\} d v+\int_{\epsilon^{\prime}}^{b} f(v)^{-1} f^{\prime}(v) d v,
$$

where we have used the fact that in a given variable, the rule for subtraction in a logarithmically divergent integral is to replace the lower limit of integration by $\epsilon$ instead of 0 . Using

$$
\int_{\epsilon}^{a} \frac{d u}{u}=\int_{\epsilon^{\prime}}^{b} f(v)^{-1} f^{\prime}(v) d v
$$

we get

$$
J^{\prime}=J-\ln a_{0} .
$$

It is worth noting that the origins of the errors (3.14) and (3.19) are somewhat different. The error (3.14) associated with linearly divergent integral arises due to the fact that under a change of variable from $u$ to $f(u)$, the replacement rule (3.4) does not remain invariant but gets shifted by a constant. However, since the right hand sides of (3.5) and (3.10) are independent of the cut-offs $\epsilon$ and $\epsilon^{\prime}$, the error is not related to a change in the cut-off. On the other hand the error (3.19) arises from the fact that for logarithmically divergent integral the integration region $u \leq \epsilon$ is removed by hand, making the integral dependent 
on the cut-off $\epsilon$. Therefore if we make a change of variable from $u$ to $v$ and use the same cut-off $\epsilon$ for the $v$ variable, then it corresponds to a different cut-off in the original variable $u$ and result changes.

Eqs. (3.14) and (3.19) show that if we do not want to make an error in the evaluation of an amplitude, then for a linearly divergent integral we are allowed to make a fractional error of order $u^{2}$, while for a logarithmically divergent integral we are allowed to make a fractional error of order $u$. Intuitively these results may be understood as follows. When there is a tachyon in the intermediate state, it can produce a contribution of order $1 / \epsilon$ in the integral according to (3.4). The $1 / \epsilon$ term is expected to cancel at the end, leaving behind a finite contribution. However a fractional error of order $\epsilon$ can combine with the $1 / \epsilon$ term to give a finite contribution that does not cancel. In contrast, divergences associated with massless particle poles are logarithmic. Therefore a fractional error of order $\epsilon$ is not important, but the leading order identification of variables must be correct.

Even though we have not carried out a detailed analysis for the many variable case, we shall adopt this guideline for determining what are allowed approximations. Regarding the $\epsilon_{i}$ 's associated with different degenerations as independent, we can see that in general we cannot ignore fractional errors of order $\epsilon_{i}$, but can ignore higher order corrections. Thus, for example, for two variables we shall not ignore terms of order $\epsilon_{1}, \epsilon_{2}$ or $\epsilon_{1} \epsilon_{2}$ but will ignore terms of order $\epsilon_{1}^{2}$ and $\epsilon_{2}^{2}$. One might worry that $1 / \epsilon_{1} \epsilon_{2}$ contribution from the tachyon may combine with the $\epsilon_{1}^{2}$ term to give a finite contribution $\epsilon_{1} / \epsilon_{2}$. However since we know that the final results are independent of $\epsilon_{1}$ and $\epsilon_{2}$, such terms must cancel at the end. If in some cases $\epsilon_{1}$ and $\epsilon_{2}$ cannot be taken to be independent - since they are determined by the underlying string field theory - then we need to be a bit more careful and keep terms up to sufficient order in order not to make an error.

In our analysis, the $\epsilon_{i}$ 's will be determined in terms of two string field theory parameters $\tilde{\lambda}$ and $\alpha$, which we shall take to be large. The contributions from different Feynman diagrams will have the structure of Laurent series expansion in $\alpha^{-1}$ and $\tilde{\lambda}^{-1}$. We shall drop terms with positive powers of $\alpha^{-1}$ and / or $\widetilde{\lambda}^{-1}$ by taking $\alpha$ and $\tilde{\lambda}$ to be large. Since the final result is expected to be independent of $\alpha$ and $\widetilde{\lambda}$, we could in principle also drop terms with positive powers of $\alpha$ and / or $\tilde{\lambda}$, keeping only the terms that are independent of $\alpha$ and $\widetilde{\lambda}$. However we keep these terms, since their cancellation in the final result provides a consistency test of our results.

Finally, we note that the results of this section are also valid if we use the alternative procedure described in section 2.4. To see this, recall that the procedure of section 2.4 involves dropping terms proportional to $\delta^{-1}$ and $\ln \delta$ from the boundary at $q=\delta$ before taking the $\delta \rightarrow 0$ limit. Now if we make a change of variable from $q$ to $\tilde{q}=b_{0} q+c_{0} q^{2}+$ $\mathcal{O}\left(q^{3}\right)$, then the boundary at $q=\delta$ corresponds to $\tilde{q}=\tilde{\delta}$ were $\tilde{\delta}=b_{0} \delta+c_{0} \delta^{2}+\mathcal{O}\left(\delta^{3}\right)$. Therefore, if we were using the variable $\tilde{q}$ as the sewing parameter, then we would drop terms proportional to $\tilde{\delta}^{-1}$ and $\ln \tilde{\delta}$. It is easy to see that $\tilde{\delta}^{-1}=b_{0}^{-1} \delta^{-1}-c_{0} b_{0}^{-2}+\mathcal{O}(\delta)$ and $\ln \tilde{\delta}=\ln \delta+\ln b_{0}+\mathcal{O}(\delta)$. Therefore as long as $b_{0}=1$ and $c_{0}=0$, we shall not make any error if we interpret $\tilde{q}$ as the sewing parameter instead of $q$. This argument easily generalizes to the case of multiple sewing parameters. 


\section{Interaction vertices and covering of the moduli space}

The string field theory that we shall use falls in the general framework discussed in [19-21], but the actual interaction vertices that we use in our analysis will be based on the choice of local coordinates that are related to the global upper half plane coordinates by $\operatorname{SL}(2, R)$ transformation. In this section we shall describe our choice of local coordinates for defining various interaction vertices of string field theory, and also describe how the contributions from different Feynman diagrams cover different regions of the moduli space of Riemann surfaces with punctures. We shall denote an external open string by $\mathrm{O}$ and an external closed string by C. Since our goal will be to understand how to integrate over the open string fields in fixed background closed string fields, we shall only consider Feynman diagrams with internal open string propagators and not those with internal closed string propagators.

There are more elegant choice of interaction vertices based on half string overlap [34], the minimal area metric [20,21] or the hyperbolic metric [35-39], but for practical calculations the vertices based on $\operatorname{SL}(2, R)$ transformations seem to be more useful. ${ }^{4}$ Since string field theories associated with different choices of interaction vertices are related to each other by field redefinition [18], all physical results will be independent of this choice.

\subsection{C-O amplitude on the disk}

The C-O amplitude on the disk has zero dimensional moduli space, and therefore the entire contribution to the amplitude comes from the elementary C-O interaction vertex shown in figure 1(a). This can be described as a unit disk parametrized by the complex coordinate $\xi$, with a closed string puncture at the center $\xi=0$ and an open string puncture at the boundary (which we can take to be at $\xi=1$ ). We choose the local coordinates at the open string puncture to be

$$
w=i \lambda \frac{1-\xi}{1+\xi},
$$

where $\lambda$ is a large positive number. We can map the unit disk to the upper half plane labelled by $z$ via

$$
z=i \frac{1-\xi}{1+\xi}
$$

so that (4.1) takes the form

$$
w=\lambda z .
$$

In the $z$-plane the closed string puncture is located at $i$.

On the unit disk, instead of choosing the open string puncture at $\xi=1$, we could choose it at another point $\xi=\xi_{0}$ on the unit circle. Rotational invariance suggests the associated choice of local coordinate to be

$$
w=i \lambda \frac{\xi_{0}-\xi}{\xi_{0}+\xi} .
$$

In the $z$ coordinate system this translates to

$$
w=\lambda \frac{z-z_{0}}{1+z z_{0}}, \quad z_{0} \equiv i \frac{1-\xi_{0}}{1+\xi_{0}} .
$$

\footnotetext{
${ }^{4}$ See $[40]$ for the use of $\operatorname{SL}(2, C)$ vertex for the computation of one point function on the torus.
} 


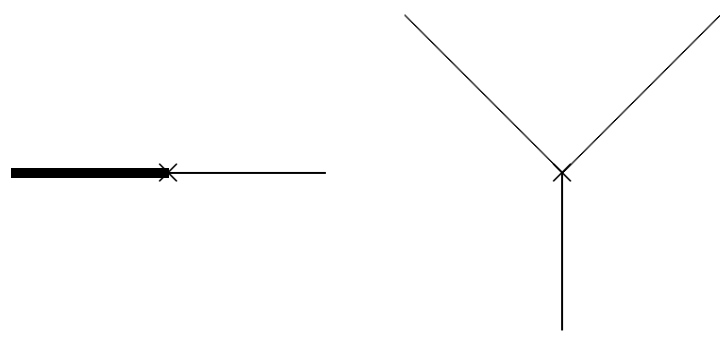

(a)

(b)

Figure 1. This figure shows two elementary interaction vertices with zero dimensional moduli space. The thick lines denote closed strings and the thin lines denote open strings. A $\times$ represents disk amplitude. Figure (a) represent open-closed disk amplitude while figure (b) represents openopen-open disk amplitude.

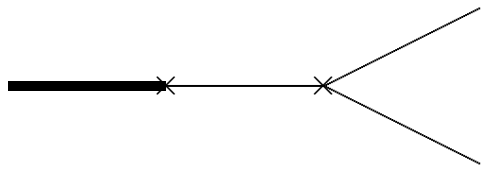

(a)

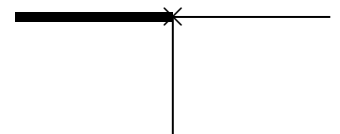

(b)

Figure 2. This figure shows two Feynman diagrams contributing to the disk amplitude with one external closed string and two external open strings.

\section{$4.2 \quad$ O-O-O amplitude on the disk}

The O-O-O amplitude on the disk also has zero dimensional moduli space, and therefore the entire contribution to the amplitude comes from the elementary O-O-O interaction vertex shown in figure 1(b). In the coordinate $z$, labelling the upper half plane, the punctures are taken to be located at 0,1 and $\infty$ with local coordinates:

$$
w_{1}=\alpha \frac{2 z}{2-z}, \quad w_{2}=-2 \alpha \frac{1-z}{1+z}, \quad w_{3}=\alpha \frac{2}{1-2 z},
$$

where $\alpha$ is a large positive number. These choices of local coordinates get cyclically permuted under the transformation $z \rightarrow 1 /(1-z)$ that cyclically permutes 0,1 and $\infty$.

\subsection{C-O-O amplitude on the disk}

The moduli space of the C-O-O amplitude on the disk is one dimensional, and can be represented by an upper half plane with the closed string puncture at $i$ and open string punctures at $\pm \beta$ with $0 \leq \beta \leq \infty$. This amplitude receives contribution from two sources, the Feynman diagram obtained by joining the C-O interaction vertex to the O-O-O interaction vertex by an open string propagator, as shown in figure 2(a), and the elementary C-O-O interaction vertex shown in figure $2(\mathrm{~b})$. Let us first consider the contribution from figure 2(a). For this we denote by $z$ the coordinate on the upper half plane associated with the $\mathrm{C}-\mathrm{O}$ interaction vertex, and by $\tilde{z}$ the coordinate on the upper half plane associated with the O-O-O interaction vertex. The Feynman rules of string field theory translate to 
the statement that the local coordinate $w$ around the open string puncture of the C-O interaction vertex, given in (4.3), should be identified with the local coordinate around one of the open string punctures of the O-O-O interaction vertex (say $w_{1}$ given in (4.6) with $z$ replaced by $\tilde{z}$ ) via the identification:

$$
w w_{1}=-q, \quad 0 \leq q \leq 1 .
$$

This gives:

$$
\widetilde{\lambda} z \frac{2 \tilde{z}}{2-\tilde{z}}=-q, \quad \tilde{\lambda} \equiv \lambda \alpha, \quad 0 \leq q \leq 1 .
$$

This gives an upper half plane labelled by $z$, with a closed string puncture located at $z=i$ and a pair of open string punctures located at $\tilde{z}=1, \infty$. In the $z$ plane, these translate to:

$$
z_{1}=-\frac{q}{2 \widetilde{\lambda}}, \quad z_{2}=\frac{q}{2 \widetilde{\lambda}}
$$

Therefore this Feynman diagram covers part of the moduli space corresponding to $\beta \leq$ $(2 \tilde{\lambda})^{-1}$. Furthermore, the local coordinates at the open string punctures are given by $w_{2}$ and $w_{3}$ in (4.6), which we shall rename as $w_{1}$ and $w_{2}$ respectively:

$$
w_{1}=-2 \alpha \frac{1-\tilde{z}}{1+\tilde{z}}=-2 \alpha \frac{z-z_{1}}{z+3 z_{1}}, \quad w_{2}=\alpha \frac{2}{1-2 \tilde{z}}=2 \alpha \frac{z-z_{2}}{z+3 z_{2}} .
$$

These can be written in a compact form as

$$
w_{a}=\frac{4 \alpha \tilde{\lambda}}{3 q} \frac{z-z_{a}}{1+\left(3 z_{a}\right)^{-1} z}, \quad a=1,2 .
$$

The C-O-O interaction vertex shown in figure $2(\mathrm{~b})$ must be defined so that it covers the moduli space corresponding to $\beta>(2 \widetilde{\lambda})^{-1}$, and at $\beta=(2 \widetilde{\lambda})^{-1}$ the choice of local coordinates at the open string punctures match the results given in (4.11) at $q=1$. We choose the local coordinates at the punctures to be

$$
w_{a}=\alpha \widetilde{\lambda} \frac{4 \widetilde{\lambda}^{2}+1}{4 \widetilde{\lambda}^{2}} \frac{z-z_{a}}{\left(1+z_{a} z\right)+\widetilde{\lambda} f\left(z_{a}\right)\left(z-z_{a}\right)}, \quad a=1,2, \quad z_{1}=-\beta, \quad z_{2}=\beta,
$$

where $f\left(z_{a}\right)$ interpolates between

$$
f\left( \pm \frac{1}{2 \widetilde{\lambda}}\right)= \pm \frac{4 \widetilde{\lambda}^{2}-3}{8 \widetilde{\lambda}^{2}} \quad \text { to } \quad f( \pm 1)=0
$$

This ensures that at $\beta=(2 \widetilde{\lambda})^{-1}$ the local coordinate of the $\mathrm{C}-\mathrm{O}-\mathrm{O}$ interaction vertex matches the local coordinate of the Feynman diagram figure 2(a) given in (4.11). The significance of the second condition in (4.13) will be discussed later. We shall further simplify the choice by taking

$$
f(-\beta)=-f(\beta) .
$$

We restrict $\beta$ to lie in the range $\beta \leq 1$ since a $z \rightarrow-1 / z$ transformation leaves the closed string puncture at $i \infty$ fixed and exchanges the range $0<\beta<1$ with $1<\beta<\infty$. This 
Figure 3. This figure shows two Feynman diagrams contributing to the disk amplitude with two external closed strings.

also exchanges the positions of the two open string punctures and their local coordinates. Therefore once we restrict $\beta$ to the range $\beta<1$, we also need to explicitly add contributions that are related by the exchange of two open string punctures. Alternatively, we could extend the range of $\beta$ all the way to $2 \tilde{\lambda}$, defining $f(\beta)=f(-1 / \beta)=-f(1 / \beta)$ for $\beta \geq 1$. In this case we do not need to separately add contributions where we exchange the two open string punctures. The condition $f( \pm 1)=0$ in (4.13) has been chosen to ensure compatibility with the $f(\beta)=-f(1 / \beta)$ condition at $\beta= \pm 1$.

Note that we could have multiplied the choice of $w_{a}$ given in (4.12) by an arbitrary function $g(\beta)$ of $\beta$ that takes value 1 at $\beta=(2 \widetilde{\lambda})^{-1}$ and satisfies the condition $g(\beta)=$ $g(-\beta)=g(1 / \beta)$, but is otherwise arbitrary. This will make the later analysis a bit more complicated. For this reason, we shall proceed with the choice $g(\beta)=1$. We shall check in appendix $\mathrm{E}$ that inclusion of such multiplicative function does not change the final results.

\section{$4.4 \quad$ C-C amplitude on the disk}

The moduli space of the C-C amplitude on the disk is one dimensional, and can be represented by an upper half plane with the first closed string puncture at $i$ and the second closed string puncture at $i y$ with $0<y<1$. This amplitude receives contribution from two sources, the Feynman diagram obtained by joining the C-O interaction vertex to the $\mathrm{C}-\mathrm{O}$ interaction vertex by an open string propagator, as shown in figure 3(a), and the elementary C-C interaction vertex shown in figure $3(\mathrm{~b})$. Let us first consider the contribution from figure $3(\mathrm{a})$. If we denote by $z$ and $\hat{z}$ the coordinates on the upper half planes associated with the two C-O vertices, then as a consequence of the local coordinate choice (4.3), the two disks are glued via the relation

$$
\lambda z \lambda \hat{z}=-q, \quad 0 \leq q \leq 1 .
$$

This maps $\hat{z}=i$ to $z=i y$ with

$$
y=q / \lambda^{2} .
$$

The condition $q \leq 1$ corresponds to the following region in the $y$-space:

$$
\mathrm{A}: \quad y \leq \lambda^{-2} .
$$

Therefore the C-C interaction vertex shown in figure 3(b) must cover the region

$$
\text { B : } \quad 1 \geq y \geq \lambda^{-2} \text {. }
$$




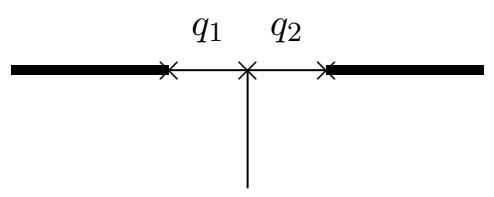

(a)

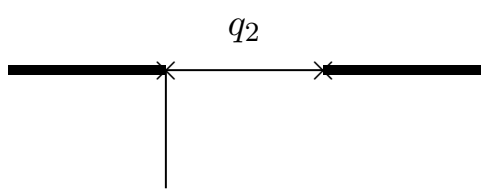

(c)

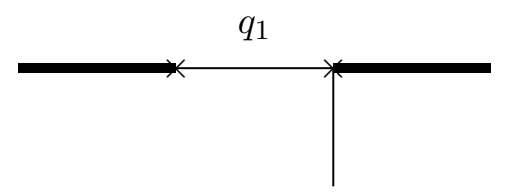

(b)

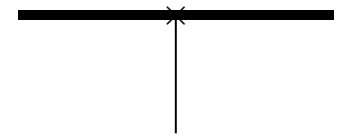

(d)

Figure 4. This figure shows four Feynman diagrams contributing to the disk amplitude with two external closed strings and one external open string. $q_{i}$ 's denote the sewing parameters of the corresponding open string propagators. They have been chosen such that when a propagator in figure(a) collapses, the parametrization agrees with the parametrization given in figure (b) or (c). With this parametrization, the $q_{1}=\delta_{1}$ boundary segments of figure (a) and figure (b) will describe a continuous curve, and the $q_{2}=\delta_{2}$ boundary segments of figure (a) and figure (c) will describe a continuous curve. This will play no role in the analysis presented in this paper, but is useful when we follow the alternative procedure described in section 2.4 and [12].

Note that the $y \geq 1$ region can be brought back to the $y \leq 1$ region by $z \rightarrow-1 / z$ transformation.

The region B actually contains a boundary of the moduli space associated with closed string degeneration, since as $y \rightarrow 1$ the two closed string punctures approach each other. In the full open-closed string field theory, we need to represent the contribution from this region as coming from a new Feynman diagram in which a C-C-C vertex is connected to a $\mathrm{C}$ vertex (one point function of the closed string on the disk) by an internal closed string propagator. This will be necessary for evaluating the full contribution to $f\left(\omega_{1}, \omega_{2}\right)$ given in (1.3) that has a singular contribution from this region [17]. However, since our goal is to evaluate $f_{\text {div }}$ given in (1.9) which does not have any divergence from the $y \rightarrow 1$ region, we do not need this special treatment of the $y \simeq 1$ region.

\subsection{C-C-O amplitude on the disk}

The moduli space of the C-C-O amplitude on the disk is two dimensional, and can be represented by an upper half plane with the first closed string puncture at $i$, the second closed string puncture at $i y$ with $0<y<1$, and the open string puncture at a point $x$ on the real axis. This amplitude receives contribution from the four Feynman diagrams shown in figure 4.

Let us begin by analyzing the Feynman diagram shown in figure 4(a) comprising the $\mathrm{C}-\mathrm{O}$, O-O-O, C-O vertices connected by a pair of open string propagators. We denote by $z$ the coordinate on the upper half plane representing the $\mathrm{C}-\mathrm{O}$ interaction vertex on the left, by $\tilde{z}$ the coordinate of the upper half plane representing the O-O-O interaction vertex, and by $\hat{z}$ the coordinate on the upper half plane representing the $\mathrm{C}-\mathrm{O}$ interaction vertex 
on the right. Using (4.3) and (4.6) we get the sewing relations to be,

$$
\tilde{\lambda} z \frac{2 \tilde{z}}{2-\tilde{z}}=-q_{1}, \quad \tilde{\lambda} \hat{z} \frac{2}{1-2 \tilde{z}}=-q_{2}, \quad \tilde{\lambda} \equiv \lambda \alpha, \quad 0 \leq q_{1}, q_{2} \leq 1 .
$$

In the $z$ plane, the location of the first closed string puncture at $z=i$, the second closed string puncture at $\hat{z}=i$ and the open string puncture at $\tilde{z}=1$ are mapped to:

$$
z_{c}^{(1)}=i, \quad z_{c}^{(2)}=-u_{1} \frac{3 u_{2}-2 i}{2\left(u_{2}+2 i\right)}, \quad z_{o}=-\frac{u_{1}}{2}, \quad u_{i}=q_{i} / \widetilde{\lambda} \quad \text { for } i=1,2 .
$$

We shall now make a coordinate transformation

$$
z=\frac{z^{\prime}+a}{-a z^{\prime}+1} \quad \Leftrightarrow \quad z^{\prime}=\frac{z-a}{a z+1},
$$

and adjust the real constant $a$ so that in the $z^{\prime}$ plane the closed string punctures are located at $i$ and $i y$ for some real number $y$. This gives

$$
i y=\frac{z_{c}^{(2)}-a}{a z_{c}^{(2)}+1} .
$$

The vanishing of the real part of the right hand side fixes $a$, which can then be substituted into the same equation to determine $y$. We shall carry out the analysis for small $u_{1}, u_{2}$. This gives:

$$
a \simeq \frac{1}{2} u_{1}\left\{1-u_{2}^{2}+\mathcal{O}\left(u_{1}^{4}, u_{2}^{4}, u_{1}^{2} u_{2}^{2}\right)\right\}
$$

and

$$
y=u_{1} u_{2}\left\{1-\frac{u_{1}^{2}}{4}-\frac{u_{2}^{2}}{4}+\mathcal{O}\left(u_{1}^{4}, u_{2}^{4}, u_{1}^{2} u_{2}^{2}\right)\right\} .
$$

Also in the $z^{\prime}$ plane the open string puncture is located at $x$ with

$$
x=-u_{1}\left\{1+\frac{u_{1}^{2}}{4}-\frac{u_{2}^{2}}{2}+\mathcal{O}\left(u_{1}^{4}, u_{2}^{4}, u_{1}^{2} u_{2}^{2}\right)\right\} .
$$

The region of the moduli space covered by this diagram corresponds to

$$
0 \leq u_{1} \leq \widetilde{\lambda}^{-1}, \quad 0 \leq u_{2} \leq \widetilde{\lambda}^{-1} .
$$

The range (4.26) translates to a region in the $x-y$ plane bounded by the following curves:

$$
\mathrm{I}: \quad x=0, \quad \frac{y}{x}=0, \quad x=-\widetilde{\lambda}^{-1}\left\{1+\frac{1}{4} \widetilde{\lambda}^{-2}-\frac{1}{2} \frac{y^{2}}{x^{2}}\right\}, \quad \frac{y}{x}=-\tilde{\lambda}^{-1}\left\{1-\frac{x^{2}}{2}+\frac{1}{4} \tilde{\lambda}^{-2}\right\},
$$

where we have neglected fractional errors of order higher than $\tilde{\lambda}^{-2}$. This can be justified as follows. We shall see in section 6.3 that the maximally divergent part of the integrand goes as $d x d y y^{-2}(x-i y)^{-1}$. Using the fact that $y \sim u_{1} u_{2} \sim \widetilde{\lambda}^{-2}$ and $x \sim u_{1} \sim \widetilde{\lambda}^{-1}$, we see that the maximally divergent part of the integral goes as $\widetilde{\lambda}^{2}$. Therefore, it is sufficient to keep fractional corrections to order $\widetilde{\lambda}^{-2}$ to extract all the finite contributions. 
There is another contribution to the diagram containing $\mathrm{C}-\mathrm{O}, \mathrm{O}-\mathrm{O}-\mathrm{O}$ and $\mathrm{C}-\mathrm{O}$ vertices, where we reverse the cyclic order of the $\mathrm{O}-\mathrm{O}-\mathrm{O}$ interaction vertex. This is equivalent to exchanging the two punctures of the O-O-O interaction vertex to which the left and the right $\mathrm{C}-\mathrm{O}$ interaction vertices are sewed. The new sewing relations take the form:

$$
\tilde{\lambda} z \frac{2}{1-2 \tilde{z}}=-q_{1}, \quad \tilde{\lambda} \hat{z} \frac{2 \tilde{z}}{2-\tilde{z}}=-q_{2}, \quad 0 \leq q_{1}, q_{2} \leq 1
$$

In the $z$ plane, the locations of the first closed string puncture at $z=i$, the second closed string puncture at $\hat{z}=i$ and the open string puncture at $\tilde{z}=1$ are given by

$$
z_{c}^{(1)}=i, \quad z_{c}^{(2)}=u_{1} \frac{3 u_{2}+2 i}{2\left(u_{2}-2 i\right)}, \quad z_{o}=\frac{u_{1}}{2}, \quad u_{i}=q_{i} / \widetilde{\lambda} \quad \text { for } i=1,2 .
$$

We now make a change of coordinate $z^{\prime}=(z-a) /(a z+1)$ so that in the $z^{\prime}$ plane the closed string punctures are located at $i$ and $i y$ for some real number $y$. This gives

$$
a \simeq-\frac{1}{2} u_{1}\left\{1-u_{2}^{2}+\mathcal{O}\left(u_{1}^{4}, u_{2}^{4}, u_{1}^{2} u_{2}^{2}\right)\right\}
$$

and

$$
y \simeq u_{1} u_{2}\left\{1-\frac{u_{1}^{2}}{4}-\frac{u_{2}^{2}}{4}+\mathcal{O}\left(u_{1}^{4}, u_{2}^{4}, u_{1}^{2} u_{2}^{2}\right)\right\} .
$$

Also in the $z^{\prime}$ plane the open string puncture is located at $x$ with

$$
x=u_{1}\left\{1+\frac{u_{1}^{2}}{4}-\frac{u_{2}^{2}}{2}+\mathcal{O}\left(u_{1}^{4}, u_{2}^{4}, u_{1}^{2} u_{2}^{2}\right)\right\} .
$$

The region of the moduli space covered by this diagrams corresponds to

$$
0 \leq u_{1} \leq \widetilde{\lambda}^{-1}, \quad 0 \leq u_{2} \leq \widetilde{\lambda}^{-1} .
$$

This translates to the region in the $x-y$ plane bounded by the curves

$$
\mathrm{I}^{\prime}: \quad x=0, \quad \frac{y}{x}=0, \quad x=\tilde{\lambda}^{-1}\left\{1+\frac{1}{4} \widetilde{\lambda}^{-2}-\frac{1}{2} \frac{y^{2}}{x^{2}}\right\}, \quad \frac{y}{x}=\tilde{\lambda}^{-1}\left\{1-\frac{x^{2}}{2}+\frac{1}{4} \tilde{\lambda}^{-2}\right\} .
$$

Next we consider the diagram shown in figure 4(b). If we denote by $z$ the coordinate on the upper half plane associated with the $\mathrm{C}-\mathrm{O}$ interaction vertex, and by $\hat{z}$ the upper half plane coordinate associated with the C-O-O interaction vertex, then, using (4.12) and (4.3), the sewing relation takes the form:

$$
\alpha \widetilde{\lambda} \frac{4 \widetilde{\lambda}^{2}+1}{4 \widetilde{\lambda}^{2}} \frac{\hat{z}+\beta}{(1-\beta \hat{z})+\widetilde{\lambda} f(-\beta)(\hat{z}+\beta)} \lambda z=-q_{1} .
$$

We also need to separately consider

$$
\alpha \widetilde{\lambda} \frac{4 \widetilde{\lambda}^{2}+1}{4 \widetilde{\lambda}^{2}} \frac{\hat{z}-\beta}{(1+\beta \hat{z})+\widetilde{\lambda} f(\beta)(\hat{z}-\beta)} \lambda z=-q_{1} .
$$


First consider the case of sewing via (4.35). In this case the second closed string puncture, located at $\hat{z}=i$, is mapped in the $z$-plane to

$$
z_{c}=u\left\{-f(-\beta)+i \widetilde{\lambda}^{-1}\right\}, \quad u \equiv \frac{4 \widetilde{\lambda} q_{1}}{4 \widetilde{\lambda}^{2}+1}, \quad 0 \leq u \leq \frac{4 \widetilde{\lambda}}{4 \widetilde{\lambda}^{2}+1},
$$

where we have used $\widetilde{\lambda}=\alpha \lambda$. We need to bring this on the imaginary axis via the change of coordinate $z^{\prime}=(z-a) /(a z+1)$ that maps the location of the first closed string puncture at $z=i$ to $z^{\prime}=i$ and the second closed string puncture at $z=z_{c}$ to $z_{c}^{\prime}=\left(z_{c}-a\right) /\left(1+a z_{c}\right)$. $a$ is determined as before by demanding that the real part of $z_{c}^{\prime}$ vanishes. This gives, using $f(-\beta)=-f(\beta)$ :

$$
a=u f(\beta)\left\{1+u^{2} \widetilde{\lambda}^{-2}+\mathcal{O}\left(u^{4} \widetilde{\lambda}^{-4}\right)\right\}
$$

and,

$$
z_{c}^{\prime}=i u \widetilde{\lambda}^{-1}\left\{1-u^{2} f(\beta)^{2}+\mathcal{O}\left(u^{4}, u^{2} \widetilde{\lambda}^{-2}\right)\right\} .
$$

We also need to find the location of the external open string puncture in the $z^{\prime}$ coordinate. In the $\hat{z}$ coordinate it is located at $\hat{z}=\beta$. Using (4.35), we get

$$
z_{o}=u\left\{f(\beta)-\frac{1-\beta^{2}}{2 \beta \widetilde{\lambda}}\right\}
$$

In the $z^{\prime}$ coordinate this is located at

$$
z_{o}^{\prime}=-\frac{u}{\widetilde{\lambda}} \frac{1-\beta^{2}}{2 \beta}\left\{1-u^{2} f(\beta)^{2}+u^{2} \widetilde{\lambda}^{-1} f(\beta) \frac{\left(1+\beta^{2}\right)^{2}}{2 \beta\left(1-\beta^{2}\right)}+\mathcal{O}\left(u^{2} \widetilde{\lambda}^{-1}\right)\right\} .
$$

Note that due to the presence of the $\widetilde{\lambda}^{-1}$ factor in the third term inside the curly bracket, this term can contribute only for $\beta \sim 1 / \widetilde{\lambda}$. Therefore we can replace the $\beta$ dependent factor multiplying this by any function of $\beta$ that approaches $1 /(2 \beta)$ for small $\beta$. We shall choose this to be $\left(1-\beta^{2}\right) /(2 \beta)$ for convenience, but we have confirmed using Mathematica that the final result remains unchanged even if we use the original form given in (4.41). Labelling $z_{c}^{\prime}$ by $i y$ and $z_{o}^{\prime}$ by $x$, and ignoring fractional corrections of order higher than $\widetilde{\lambda}^{-2}$, we can write the relations as,

$$
y=\frac{u}{\widetilde{\lambda}}\left\{1-u^{2} f(\beta)^{2}\right\}, \quad x=-\frac{u}{\widetilde{\lambda}} \frac{1-\beta^{2}}{2 \beta}\left\{1-u^{2} f(\beta)^{2}+u^{2} f(\beta) \frac{1-\beta^{2}}{2 \beta \widetilde{\lambda}}\right\} .
$$

We now see from (4.42) that the range

$$
0 \leq u \leq \frac{4 \widetilde{\lambda}}{4 \widetilde{\lambda}^{2}+1}, \quad 1 \geq \beta \geq \frac{1}{2 \widetilde{\lambda}}
$$

corresponds to the region bounded by the curves:

$$
\mathrm{II}^{\prime}: y=0, \quad y=\widetilde{\lambda}^{-2}\left\{1-F(x)^{2} \widetilde{\lambda}^{-2}-\frac{1}{4} \widetilde{\lambda}^{-2}\right\}, \quad \frac{x}{y}=0, \quad \frac{x}{y}=-\widetilde{\lambda}\left\{1-\frac{1}{4} \widetilde{\lambda}^{-2}+\frac{1}{2} x^{2}\right\},
$$


where $F(x)$ is a function of $x$ defined as the result of elimination of $\beta$ from the equations:

$$
\begin{aligned}
& x=-\frac{1}{\widetilde{\lambda}^{2}} \frac{1-\beta^{2}}{2 \beta}\left\{1-\frac{1}{4} \tilde{\lambda}^{-2}-\tilde{\lambda}^{-2} f(\beta)^{2}+\tilde{\lambda}^{-2} f(\beta) \frac{1-\beta^{2}}{2 \beta \widetilde{\lambda}}\right\}, \quad F(x)=f(\beta), \\
& \frac{1}{2 \widetilde{\lambda}} \leq \beta \leq 1 \quad \Leftrightarrow \quad-\widetilde{\lambda}^{-1}\left(1-\frac{1}{4 \widetilde{\lambda}^{2}}\right) \leq x \leq 0 .
\end{aligned}
$$

The case of sewing via (4.36) can be analyzed similarly, and yields the result:

$$
y=\frac{u}{\widetilde{\lambda}}\left\{1-u^{2} f(\beta)^{2}\right\}, \quad x=\frac{u}{\widetilde{\lambda}} \frac{1-\beta^{2}}{2 \beta}\left\{1-u^{2} f(\beta)^{2}+u^{2} f(\beta) \frac{1-\beta^{2}}{2 \beta \widetilde{\lambda}}\right\},
$$

The range of $x$ and $y$ covered by this diagram is bounded by:

$$
\mathrm{II}: y=0, \quad y=\widetilde{\lambda}^{-2}\left\{1-F(x)^{2} \widetilde{\lambda}^{-2}-\frac{1}{4} \widetilde{\lambda}^{-2}\right\}, \quad \frac{x}{y}=0, \quad \frac{x}{y}=\widetilde{\lambda}\left\{1-\frac{1}{4} \widetilde{\lambda}^{-2}+\frac{1}{2} x^{2}\right\},
$$

where, $F(x)$ is now defined via:

$$
\begin{aligned}
& x=\frac{1}{\widetilde{\lambda}^{2}} \frac{1-\beta^{2}}{2 \beta}\left\{1-\frac{1}{4} \widetilde{\lambda}^{-2}-\widetilde{\lambda}^{-2} f(\beta)^{2}+\widetilde{\lambda}^{-2} f(\beta) \frac{1-\beta^{2}}{2 \beta \widetilde{\lambda}}\right\}, \quad F(x)=f(\beta), \\
& \frac{1}{2 \widetilde{\lambda}} \leq \beta \leq 1 \quad \Leftrightarrow \quad 0 \leq x \leq \widetilde{\lambda}^{-1}\left(1-\frac{1}{4 \widetilde{\lambda}^{2}}\right) .
\end{aligned}
$$

Next we consider the diagram shown in figure 4(c). This is related to the contribution from figure 4(b) by the exchange of the two closed string punctures. In the $z^{\prime}$ plane this can be achieved by the transformation $z^{\prime} \rightarrow-y / z^{\prime}$, taking the point $i$ to $i y$ and the point $i y$ to $i$. On the open string punctures located at $z^{\prime}=x$, this has the effect of sending $x$ to $-y / x$. Therefore the equations analogous to (4.42) and (4.46) take the form:

$$
y=\frac{u}{\widetilde{\lambda}}\left\{1-u^{2} f(\beta)^{2}\right\}, \quad x=\frac{2 \beta}{1-\beta^{2}}\left\{1-u^{2} f(\beta) \frac{1-\beta^{2}}{2 \beta \widetilde{\lambda}}\right\},
$$

and

$$
y=\frac{u}{\widetilde{\lambda}}\left\{1-u^{2} f(\beta)^{2}\right\}, \quad x=-\frac{2 \beta}{1-\beta^{2}}\left\{1-u^{2} f(\beta) \frac{1-\beta^{2}}{2 \beta \widetilde{\lambda}}\right\} .
$$

The range of $u$ and $\beta$ given in (4.43) correspond to the regions in the $x$-y plane bounded by the curves:

$$
\mathrm{III}^{\prime}: \quad y=0, \quad y=\widetilde{\lambda}^{-2}\left\{1-F(x)^{2} \widetilde{\lambda}^{-2}-\frac{1}{4 \widetilde{\lambda}^{2}}\right\}, \quad x=\infty, \quad x=\widetilde{\lambda}^{-1}\left\{1+\frac{1}{4 \widetilde{\lambda}^{2}}-\frac{1}{2} \frac{y^{2}}{x^{2}}\right\},
$$

and

III $: \quad y=0, \quad y=\widetilde{\lambda}^{-2}\left\{1-F(x)^{2} \widetilde{\lambda}^{-2}-\frac{1}{4 \widetilde{\lambda}^{2}}\right\}, \quad x=-\infty, \quad x=-\widetilde{\lambda}^{-1}\left\{1+\frac{1}{4 \widetilde{\lambda}^{2}}-\frac{1}{2} \frac{y^{2}}{x^{2}}\right\}$, 


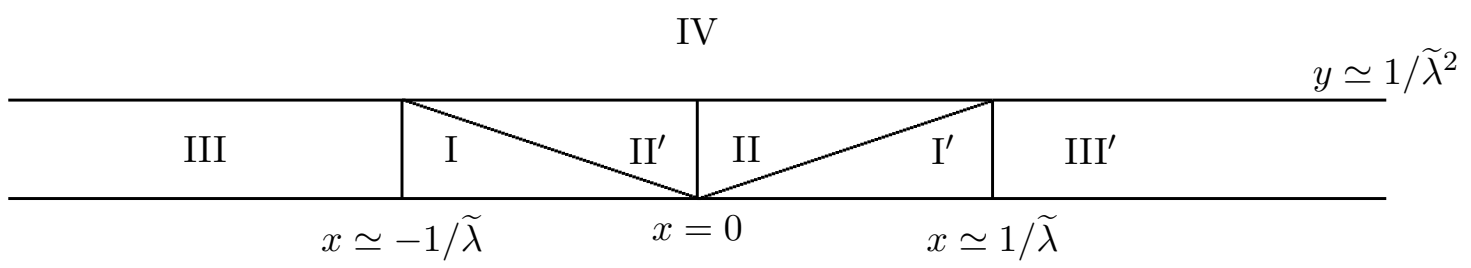

Figure 5. This figure shows the covering of the moduli space of C-C-O amplitude on the disk by various Feynman diagrams. The horizontal axis labels $x$ - the position of the open string puncture on the real axis and the vertical axis labels $y$, the position of the second closed string puncture on the imaginary axis. The first closed string puncture is situated at $i$. We use only the leading order results for large $\widetilde{\lambda}$ to plot the boundaries of various regions; the full expressions may be found in the text.

where $F(x)$ is defined via the equation:

$$
\begin{gathered}
x= \pm \frac{2 \beta}{1-\beta^{2}}\left\{1-\widetilde{\lambda}^{-2} f(\beta) \frac{1-\beta^{2}}{2 \beta \widetilde{\lambda}}\right\}, \quad F(x)=f(\beta), \\
\frac{1}{2 \widetilde{\lambda}} \leq \beta \leq 1 \quad \Leftrightarrow \quad \widetilde{\lambda}^{-1}\left(1-\frac{1}{4 \widetilde{\lambda}^{2}}\right) \leq|x|<\infty .
\end{gathered}
$$

Eqs. (4.45), (4.48), (4.53) define the function $F(x)$ over the entire real $x$-axis.

A property of the function $F(x)$ that will be useful later is that to leading order in the expansion in powers of $\widetilde{\lambda}^{-1}, F(x)$ satisfies the equation:

$$
F\left(\tilde{\lambda}^{-2} / x\right)=F(x) .
$$

Since $F(x)=f(\beta)$ for all values of $x$, to prove (4.54) we need to verify that the leading order relation between $x$ and $\beta$ remains unchanged under $x \rightarrow \widetilde{\lambda}^{-2} / x$. Now, ignoring fractional corrections of order $\widetilde{\lambda}^{-2}$, we see that for $|x|>\widetilde{\lambda}^{-1}$, the relation between $x$ and $\beta$ given in (4.53) takes the from $|x|=2 \beta /\left(1-\beta^{2}\right)$, whereas for $|x| \leq \widetilde{\lambda}^{-1}$, the relation between $x$ and $\beta$ given in (4.45), (4.48) takes the from $|x|=\widetilde{\lambda}^{-2}\left(1-\beta^{2}\right) /(2 \beta)$. Since these are related by $x \rightarrow \widetilde{\lambda}^{-2} / x$ transformation, this establishes (4.54).

The rest of the moduli space must be covered by the C-C-O interaction vertex shown in figure $4(\mathrm{~d})$. This corresponds to the region:

$$
\text { IV }: \quad 1 \geq y \geq \tilde{\lambda}^{-2}\left\{1-F(x)^{2} \widetilde{\lambda}^{-2}-\frac{1}{4} \widetilde{\lambda}^{-2}\right\}, \quad-\infty<x<\infty .
$$

Figure 5 shows the regions in the $x-y$ plane given in eqs. (4.27), (4.34), (4.44), (4.47), (4.51), (4.52) and (4.55).

\subsection{O amplitude on the annulus}

Next we consider the one point amplitude of an open string on the annulus. We shall label the annulus by the complex coordinate $w$ with the identification

$$
0 \leq \operatorname{Re} w \leq \pi, \quad w \equiv w-i \ln v
$$




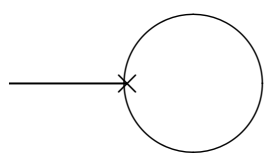

(a) (b)

Figure 6. This figure shows the Feynman diagrams contributing to the annulus amplitude with one external open string. The interaction vertex with $\mathrm{a} \times$ represents a disk amplitude, while a interaction vertex with $\otimes$ represents part of an annulus amplitude.

where $v$ is a positive real number taking value in the range $0<v<1$. The location of the open string puncture can be fixed at any arbitrary point on the boundary using the shift symmetry $\operatorname{Im}(w) \rightarrow \operatorname{Im}(w)+$ constant. Therefore the moduli space is one dimensional, parametrized by $v$.

The amplitude receives contribution from two Feynman diagrams, shown in figure 6 . Let us first analyze the contribution from figure 6(a). We denote by $z$ the coordinate associated with the O-O-O interaction vertex. Then, using the local coordinates of the $\mathrm{O}-\mathrm{O}-\mathrm{O}$ interaction vertex given in (4.6), we get the following identification in the $z$ plane:

$$
-2 \alpha \frac{1-z}{1+z} \equiv-q \frac{1-2 z}{2 \alpha}, \quad 0 \leq q \leq 1 .
$$

This can be expressed as

$$
z \equiv \frac{a z+b}{c z+d}
$$

where

$$
\left(\begin{array}{ll}
a & b \\
c & d
\end{array}\right)=\left(\begin{array}{cc}
\frac{1}{2} u^{1 / 2} & u^{-1 / 2}-\frac{1}{4} u^{1 / 2} \\
-\frac{1}{2} u^{1 / 2} & u^{-1 / 2}+\frac{1}{4} u^{1 / 2}
\end{array}\right), \quad u \equiv q / \alpha^{2}, \quad 0 \leq u \leq \alpha^{-2} .
$$

We can 'diagonalize' this matrix by defining new coordinate $\hat{z}$ via

$$
z=\frac{\left(4+3 u+\mathcal{O}\left(u^{2}\right)\right) \hat{z}-4+3 u+\mathcal{O}\left(u^{2}\right)}{\left(4+u+\mathcal{O}\left(u^{2}\right)\right) \hat{z}-2 u+\mathcal{O}\left(u^{3}\right)}, \quad w=\frac{1}{i} \ln \hat{z}, \quad 0 \leq \operatorname{Re} w \leq \pi .
$$

Then the identification (4.58) takes the form

$$
\hat{z}=u^{-1}\left\{1-\frac{1}{2} u+\mathcal{O}\left(u^{2}\right)\right\} \hat{z}, \quad w \equiv w-i \ln \left[u\left\{1-\frac{1}{2} u+\mathcal{O}\left(u^{2}\right)\right\}^{-1}\right] .
$$

Comparison with (4.56) gives

$$
v=u\left\{1-\frac{1}{2} u+\mathcal{O}\left(u^{2}\right)\right\}^{-1} .
$$

This agrees with the result of [40]. Since according to the result of section 3 , fractional errors of order $u^{2}$ can be absorbed into a redefinition of $u$ without changing the final result, we shall use the relation

$$
v=u\left\{1-\frac{1}{2} u\right\}^{-1}
$$


Therefore the range of $v$ spanned by figure 6(a) corresponds to

$$
0 \leq v \leq\left\{\alpha^{2}-\frac{1}{2}\right\}^{-1}
$$

In this coordinate system the open string puncture located at $z=0$ is mapped to,

$$
\hat{z}=1-\frac{3}{2} u+\mathcal{O}\left(u^{2}\right) \simeq 1, \quad w=i\left\{\frac{3}{2} u+\mathcal{O}\left(u^{2}\right)\right\} .
$$

The local coordinate at the open string puncture is given by

$$
w_{o}=\alpha \frac{2 z}{2-z}=2 \alpha \frac{(4+3 u) \hat{z}-4+3 u+\mathcal{O}\left(u^{2}\right)}{(4-u) \hat{z}+4-7 u+\mathcal{O}\left(u^{2}\right)} .
$$

Since translational invariance along the imaginary $w$ axis allows us to change this to any arbitrary imaginary value, the location of the open string puncture is not a modulus. Nevertheless, for definiteness, we shall stick to (4.65), (4.66).

The contribution from figure $6(\mathrm{~b})$, corresponding to the open string one point interaction vertex on the annulus, will have to span the range

$$
\left\{\alpha^{2}-\frac{1}{2}\right\}^{-1}<v<1
$$

We can choose the local coordinate at the open string puncture to be the same as the one given in (4.66) for $u=\alpha^{-2}$, i.e.

$$
w_{o}=2 \alpha \frac{\left(4+3 \alpha^{-2}\right) \hat{z}-4+3 \alpha^{-2}}{\left(4-\alpha^{-2}\right) \hat{z}+4-7 \alpha^{-2}} .
$$

We could make more general choice of $w_{o}$, e.g. include in the definition of $w_{o}$ an arbitrary multiplicative function $h(v)$ that takes value 1 at $v=1 /\left(\alpha^{2}-\frac{1}{2}\right)$ but is otherwise arbitrary. In our analysis below, this will have the effect of replacing $u$ by $u / h(v)$ in (4.86), and multiplying the upper limit of $x$ in (4.88) and the lower limit of $x$ in (4.102) by $1 / h(v)$. This in turn will affect the integrals $I_{(b)}$ and $I_{(d)}$ in (6.11) and (6.5) respectively. It is easy to verify that these changes cancel each other and do not affect the final result.

\section{7 $\quad$ C amplitude on the annulus}

Next we consider the one point amplitude of a closed string on the annulus. The moduli space for this amplitude is two dimensional. We shall label the annulus by the coordinate $w$ with the identification

$$
0 \leq \operatorname{Re} w \leq \pi, \quad w \equiv w-i \ln v,
$$

and the closed string puncture will be taken to be located at $w_{c}$ with

$$
\operatorname{Re} w_{c}=2 \pi x \text {. }
$$

The pair $(v, x)$ label the moduli on the Riemann surface, with the full range given by:

$$
0<v<1, \quad 0 \leq x \leq \frac{1}{4} .
$$




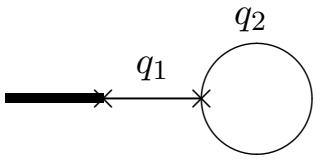

(a)

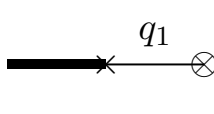

(b)

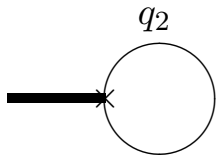

(c) (d)

Figure 7. This figure shows the Feynman diagrams contributing to the annulus amplitude with one external closed string. The interaction vertex with a $\times$ represents part of a disk amplitude, while the interaction vertex with $\otimes$ represents annulus amplitude. As in figure 4 , the $q_{i}$ 's represent the sewing parameters of the corresponding propagators.

The range $x>\frac{1}{4}$ is related to the region described above by $w \rightarrow \pi-w$ transformation. The imaginary part of $w_{c}$ can be shifted using the translation invariance of the annulus along the periodically identified imaginary $w$ axis, and therefore does not represent a modulus.

Figure 7 shows the different Feynman diagrams contributing to this amplitude. Our goal will be to find out the relation between the sewing parameters $\left\{q_{i}\right\}$ associated with these diagrams and the variables $v, x$ introduced above. Since we shall derive approximate relations between these quantities instead of exact relations, we need to make sure that we do not throw away terms that could give finite contribution to the amplitude. For this we note the following facts:

1. The relations we are after depend on two string field theory parameters $\tilde{\lambda}$ and $\alpha$ which are both taken to be large, and the amplitude, to be evaluated in section 6.1 , will be given as a Laurent series expansion in $\alpha^{-2}$ and $\tilde{\lambda}^{-1}$. In this expansion we shall keep all terms that contain non-negative power of $\alpha^{2}$ and $\tilde{\lambda}$, but will throw away terms containing negative powers of $\alpha^{2}$ or $\tilde{\lambda}$, even if they contain positive power of the other variable. The reason for this is that the $\alpha$ and $\widetilde{\lambda}$ dependent terms are expected to cancel at the end anyway, and once a term has got negative power of one large variable, there is no way it can give a $\alpha$ and $\tilde{\lambda}$ independent term at higher order in the expansion.

2. The most divergent term in the integrand that we shall encounter during our analysis in section 6.1, where these results will be used, goes as $d v d x x^{-2} v^{-2}$ for small $v, x$.

We begin by analyzing the contribution from figure 7 (a). We denote by $z$ the coordinate on the $\mathrm{C}-\mathrm{O}$ interaction vertex, and by $\tilde{z}$ the coordinate on the O-O-O interaction vertex. Then, using (4.3) and (4.6), the sewing relation takes the form:

$$
\tilde{\lambda} z \frac{2 \tilde{z}}{2-\tilde{z}}=-q_{1}, \quad-2 \alpha \frac{1-\tilde{z}}{1+\tilde{z}} \equiv-q_{2} \frac{1-2 \tilde{z}}{2 \alpha}, \quad 0 \leq q_{1}, q_{2} \leq 1 .
$$

We shall define:

$$
u_{1} \equiv q_{1} / \widetilde{\lambda}, \quad u_{2}=q_{2} / \alpha^{2}, \quad 0 \leq u_{1} \leq \widetilde{\lambda}^{-1}, \quad 0 \leq u_{2} \leq \alpha^{-2} .
$$

The second equation of (4.72) now gives

$$
\tilde{z} \equiv \frac{a \tilde{z}+b}{c \tilde{z}+d},
$$


where,

$$
\left(\begin{array}{ll}
a & b \\
c & d
\end{array}\right)=\left(\begin{array}{cc}
\frac{1}{2} u_{2}^{1 / 2} & u_{2}^{-1 / 2}-\frac{1}{4} u_{2}^{1 / 2} \\
-\frac{1}{2} u_{2}^{1 / 2} & u_{2}^{-1 / 2}+\frac{1}{4} u_{2}^{1 / 2}
\end{array}\right)
$$

As in (4.60), if we define new coordinate $\hat{z}, w$ via

$$
\tilde{z}=\frac{\left(4+3 u_{2}\right) \hat{z}-4+3 u_{2}+\mathcal{O}\left(u_{2}^{2}\right)}{\left(4+u_{2}\right) \hat{z}-2 u_{2}+\mathcal{O}\left(u_{2}^{2}\right)}, \quad w=\frac{1}{i} \ln \hat{z}, \quad 0 \leq \operatorname{Re} w \leq \pi,
$$

then the identification (4.74) takes the form

$$
\hat{z}=u_{2}^{-1}\left\{1-\frac{1}{2} u_{2}+\mathcal{O}\left(u_{2}^{2}\right)\right\} \hat{z}, \quad w \equiv w-i \ln \left[u_{2}\left\{1+\frac{1}{2} u_{2}+\mathcal{O}\left(u_{2}^{2}\right)\right\}\right] .
$$

In the $\tilde{z}$ plane, the closed string puncture at $z=i$ is at

$$
\tilde{z}_{c}=\frac{2 i u_{1}}{2+i u_{1}} .
$$

In the $w$ coordinate system this maps to

$$
w_{c}=u_{1}\left\{1-u_{2}+\mathcal{O}\left(u_{2}^{2}\right)\right\}+\mathcal{O}\left(u_{1}^{3}\right),
$$

up to addition of imaginary terms which can be removed using translational invariance along the imaginary $w$ direction. Comparison of (4.77), (4.79) with (4.69), (4.70) gives

$$
v=u_{2}\left\{1-\frac{1}{2} u_{2}+\mathcal{O}\left(u_{2}^{2}\right)\right\}^{-1}, \quad x=\frac{1}{2 \pi} u_{1}\left\{1-u_{2}+\mathcal{O}\left(u_{2}^{2}\right)\right\}+\mathcal{O}\left(u_{1}^{3}\right)
$$

Eq. (4.80) shows that for small $v, x$ the most divergent part $d x d v x^{-2} v^{-2}$ of the integrand goes as $d u_{1} d u_{2} u_{1}^{-2} u_{2}^{-2}$. It then follows from the analysis of section 3 that it will be sufficient to keep terms up to fractional errors of order $u_{1} \sim \widetilde{\lambda}^{-1}$ and $u_{2} \sim \alpha^{-2}$ but we can ignore fractional errors of order $u_{1}^{2} \sim \tilde{\lambda}^{-2}$ and / or $u_{2}^{2} \sim \alpha^{-4}$. With this understanding, we can replace $(4.80)$ by:

$$
v=u_{2}\left\{1-\frac{1}{2} u_{2}\right\}^{-1}, \quad x=\frac{1}{2 \pi} u_{1}\left(1-u_{2}\right)
$$

Therefore figure $7(\mathrm{a})$ covers the range

$$
0 \leq v \leq\left(\alpha^{2}-\frac{1}{2}\right)^{-1}, \quad 0 \leq 2 \pi x \leq \widetilde{\lambda}^{-1} \frac{2-v}{2+v}
$$

in the moduli space. This has been shown as region (a) in figure 8 .

We now turn to the contribution from figure $7(\mathrm{~b})$. We denote by $\hat{z}=e^{i w}$ the coordinate associated with the interaction vertex for the open string one point function on the annulus, and by $z$ the coordinate associated with the $\mathrm{C}-\mathrm{O}$ interaction vertex. Then, according to (4.67), we have the identification:

$$
\hat{z}=v^{-1} \hat{z}, \quad\left\{\alpha^{2}-\frac{1}{2}\right\}^{-1}<v<1 .
$$




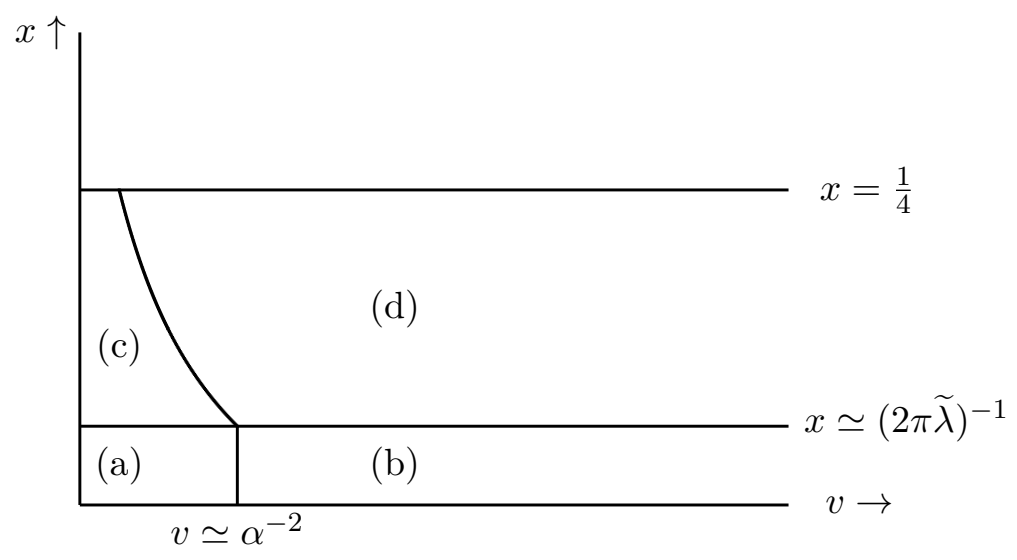

Figure 8. This figure shows the different regions of the moduli space covered by different Feynman diagrams of figure 7 . The regions are marked by keeping only the leading order term in the expansion in powers of $\alpha^{-2}$ and $\widetilde{\lambda}^{-2}$; the subleading corrections can be found in the text.

Also, using (4.3), (4.68), the sewing relation takes the form:

$$
2 \alpha \frac{\left(4+3 \alpha^{-2}\right) \hat{z}-4+3 \alpha^{-2}}{\left(4-\alpha^{-2}\right) \hat{z}+4-7 \alpha^{-2}} \lambda z=-q_{1}, \quad 0 \leq q_{1} \leq 1 .
$$

Using this we find that the closed string puncture at $z=i$ is located in the $w=\frac{1}{i} \ln \hat{z}$ plane at

$$
w_{c}=\frac{1}{i} \ln \hat{z}_{c} \simeq u\left\{1-\alpha^{-2}+\mathcal{O}\left(\alpha^{-4}\right)\right\}+\mathcal{O}\left(u^{3}\right), \quad u=\frac{q_{1}}{\widetilde{\lambda}}, \quad 0 \leq u \leq \widetilde{\lambda}^{-1} .
$$

Comparison with (4.70) gives:

$$
2 \pi x=u\left\{1-\alpha^{-2}+\mathcal{O}\left(\alpha^{-4}\right)\right\}+\mathcal{O}\left(u^{3}\right)
$$

Since $d x d v x^{-2} v^{-2}$ grows as $d u d v u^{-2} v^{-2}$ for small $u, v$, and since $v$ is bounded from below by $\alpha^{-2}$, it follows from the analysis of section 3 that we need to keep terms up to fractional errors of order $u \sim \widetilde{\lambda}^{-1}$ and $\alpha^{-2}$. Therefore we can approximate (4.86) as:

$$
2 \pi x=u\left\{1-\alpha^{-2}\right\}, \quad 0<2 \pi x<\tilde{\lambda}^{-1}\left\{1-\alpha^{-2}\right\} .
$$

Therefore figure $7(\mathrm{~b})$ covers the region:

$$
\left\{\alpha^{2}-\frac{1}{2}\right\}^{-1}<v<1, \quad 0<2 \pi x<\tilde{\lambda}^{-1}\left\{1-\alpha^{-2}\right\} .
$$

This has been shown as region (b) in figure 8 .

Next we turn to the contribution from figure $7(\mathrm{c})$. We denote by $z$ the coordinate on the $\mathrm{C}-\mathrm{O}-\mathrm{O}$ interaction vertex with the closed string puncture located at $z=i$. Then the sewing relation gives the identification

$$
w_{1}=-q_{2} / w_{2}
$$


where $w_{1}$ and $w_{2}$ have been defined in (4.12). Denoting $f(\beta)$ by $f$ for notational simplicity and using the relation $f(-\beta)=-f(\beta)$, we can express (4.89) as

$$
z \equiv \frac{a z+b}{c z+d}
$$

where

$$
\begin{aligned}
& a=\widetilde{\lambda}\left(1+\beta^{2}\right)^{-1}\left[\beta u^{-1 / 2}+u^{1 / 2} \widetilde{\lambda}^{-2}\{1-\tilde{\lambda} f \beta\}\{\widetilde{\lambda} f+\beta\}\right], \\
& b=\widetilde{\lambda}\left(1+\beta^{2}\right)^{-1}\left[\beta^{2} u^{-1 / 2}-u^{1 / 2} \widetilde{\lambda}^{-2}\{1-\widetilde{\lambda} f \beta\}^{2}\right], \\
& c=\widetilde{\lambda}\left(1+\beta^{2}\right)^{-1}\left[u^{-1 / 2}-u^{1 / 2} \widetilde{\lambda}^{-2}\{\widetilde{\lambda} f+\beta\}^{2}\right], \\
& d=\widetilde{\lambda}\left(1+\beta^{2}\right)^{-1}\left[\beta u^{-1 / 2}+u^{1 / 2} \widetilde{\lambda}^{-2}\{\tilde{\lambda} f+\beta\}\{1-\tilde{\lambda} f \beta\}\right], \\
& f \equiv f(\beta),
\end{aligned}
$$

and

$$
u=q_{2} \alpha^{-2}\left\{1+\frac{1}{4 \widetilde{\lambda}^{2}}\right\}^{-2}, \quad 0 \leq u \leq \alpha^{-2}\left\{1+\frac{1}{4 \widetilde{\lambda}^{2}}\right\}^{-2} .
$$

The transformation (4.90) can be 'diagonalized' using new coordinate $\tilde{z}$, defined via

$$
\begin{aligned}
z & =\frac{A \tilde{z}+B}{C \tilde{z}+D}, \quad \frac{A}{C}=\beta\left[1+u \widetilde{\lambda}^{-2}\left\{\frac{1+\beta^{2}}{\beta} \widetilde{\lambda} f-\frac{1-\beta^{4}}{2 \beta^{2}}\right\}+\mathcal{O}\left(u^{2}\right)\right], \\
\frac{B}{D} & =-\beta\left[1+u \widetilde{\lambda}^{-2}\left\{\frac{1+\beta^{2}}{\beta} \widetilde{\lambda} f-\frac{1-\beta^{4}}{2 \beta^{2}}\right\}+\mathcal{O}\left(u^{2}\right)\right] .
\end{aligned}
$$

We also define

$$
w=\frac{1}{i} \ln \tilde{z} .
$$

In these variables (4.90) takes the form:

$$
\begin{aligned}
\tilde{z} & \equiv\left\{\frac{4 \beta^{2} \widetilde{\lambda}^{2}}{\left(1+\beta^{2}\right)^{2} u}+8 \beta \frac{(\beta+\tilde{\lambda} f)(1-\beta \tilde{\lambda} f)}{\left(1+\beta^{2}\right)^{2}}-2+\mathcal{O}(u)\right\} \tilde{z}, \\
w & \equiv w+i \ln \left\{\frac{4 \beta^{2} \widetilde{\lambda}^{2}}{\left(1+\beta^{2}\right)^{2} u}+8 \beta \frac{(\beta+\widetilde{\lambda} f)(1-\beta \tilde{\lambda} f)}{\left(1+\beta^{2}\right)^{2}}-2+\mathcal{O}(u)\right\} .
\end{aligned}
$$

Also, in this variable the closed string puncture at $z=i$ is located at

$$
\tilde{z}_{c}=-\frac{D+i B}{C+i A}, \quad w_{c}=\pi+\tan ^{-1} \frac{B}{D}-\tan ^{-1} \frac{A}{C}+\text { imaginary } .
$$

Comparison of (4.95), (4.96) with (4.69), (4.70), and use of the $x \rightarrow \frac{1}{2}-x$ symmetry, gives

$$
\begin{aligned}
2 \pi x & =2 \tan ^{-1} \beta+2 u f \widetilde{\lambda}^{-1}-\frac{1}{\beta}\left(1-\beta^{2}\right) \widetilde{\lambda}^{-2} u+\mathcal{O}\left(u^{2}\right), \\
v & =u \frac{\left(1+\beta^{2}\right)^{2}}{4 \beta^{2} \widetilde{\lambda}^{2}}\left\{1+u \widetilde{\lambda}^{-2} \frac{1}{2 \beta^{2}}\left(1-\beta^{2}-2 \beta \widetilde{\lambda} f\right)^{2}+\mathcal{O}\left(u^{2}\right)\right\} .
\end{aligned}
$$


Since $d v d x v^{-2} x^{-2} \sim d u d \beta u^{-2} \widetilde{\lambda}^{2}$ for small $u$, we can ignore fractional errors of order $u^{2}$, since they will generate corrections containing inverse powers of $\alpha^{2}$. Therefore we can write (4.97) as

$$
\begin{aligned}
2 \pi x & =2 \tan ^{-1} \beta+2 u f \widetilde{\lambda}^{-1}-\frac{1}{\beta}\left(1-\beta^{2}\right) \tilde{\lambda}^{-2} u, \\
v & =u \frac{\left(1+\beta^{2}\right)^{2}}{4 \beta^{2} \widetilde{\lambda}^{2}}\left\{1+u \widetilde{\lambda}^{-2} \frac{1}{2 \beta^{2}}\left(1-\beta^{2}-2 \beta \widetilde{\lambda} f\right)^{2}\right\} .
\end{aligned}
$$

The range

$$
\frac{1}{2 \widetilde{\lambda}} \leq \beta \leq 1, \quad 0 \leq u \leq \alpha^{-2}\left\{1+\frac{1}{4 \widetilde{\lambda}^{2}}\right\}^{-2}
$$

translates to the region bounded by the curves:

$$
\begin{aligned}
& x=\frac{1}{\pi} \tan ^{-1}\left[\frac{1}{2 \widetilde{\lambda}}(1-v)\right], \quad x=\frac{1}{4}, \quad v=0 \\
& v=\frac{1}{\alpha^{2} \widetilde{\lambda}^{2} \sin ^{2}(2 \pi x)}\left(1+\frac{1}{4 \widetilde{\lambda}^{2}}\right)^{-2}\left[1-2\left\{\cot ^{2}(2 \pi x)-\widetilde{\lambda}^{2} f^{2}\right\} \alpha^{-2} \widetilde{\lambda}^{-2}\left(1+\frac{1}{4 \widetilde{\lambda}^{2}}\right)^{-2}\right]
\end{aligned}
$$

where now $f \equiv f(\tan (\pi x))$ and we have used

$$
f\left(\frac{1}{2 \widetilde{\lambda}}\right)=\frac{1}{2}\left\{1-\frac{3}{4 \widetilde{\lambda}^{2}}\right\}, \quad f(1)=0 .
$$

The region (4.100) has been shown as region (c) in figure 8.

The contribution from figure $7(\mathrm{~d})$ should cover the left-over region in the $x-v$ space:

$$
\begin{aligned}
& \frac{\pi}{2} \geq 2 \pi x \geq \widetilde{\lambda}^{-1}\left(1-\alpha^{-2}\right) \\
& \frac{1}{\alpha^{2} \widetilde{\lambda}^{2} \sin ^{2}(2 \pi x)}\left(1+\frac{1}{4 \widetilde{\lambda}^{2}}\right)^{-2}\left[1-2\left\{\cot ^{2}(2 \pi x)-\widetilde{\lambda}^{2} f^{2}\right\} \alpha^{-2} \widetilde{\lambda}^{-2}\left(1+\frac{1}{4 \widetilde{\lambda}^{2}}\right)^{-2}\right] \leq v<1 \\
& f \equiv f(\tan (\pi x))
\end{aligned}
$$

This has been shown as region (d) in figure 8. Eq. (4.102) is an approximate description of the left-over region, but the arguments given earlier ensure that the error due to this approximation affects the final integral only by terms containing inverse powers of $\alpha^{2}$ or $\widetilde{\lambda}$.

The regions (b) and (d) in figure 8 contain the region $v=1$. This is actually a boundary of the moduli space associated with closed string degeneration, where the annulus becomes an infinitely long cylinder. In the full open-closed string field theory, we need to represent the contribution from this region as coming from new Feynman diagrams containing internal closed string propagators. This will be necessary for evaluating the full contribution to $g(\omega)$ given in (1.5) that has a singular contribution from this region [17]. However, since our goal is to evaluate $g_{\text {div }}(\omega)$ given in (1.11) which does not have any divergence from the $v \rightarrow 1$ region, we do not need this special treatment of the $v \simeq 1$ region. 


\section{Disk two point function in two dimensional string theory}

Our analysis so far applies to any string theory D-instanton. Now we shall apply the results to the specific case of D-instantons in two dimensional string theory, whose worldsheet matter theory contains a free scalar describing the time coordinate and the Liouville theory with $c=25$. As discussed in section 1, we shall first analyze the building blocks of the amplitude, leaving out the energy conserving delta function and the $\mathcal{N} e^{-1 / g_{s}}$ factor that are common to all amplitudes.

In this section we shall analyze the building block corresponding to the disk two point function of a pair of closed string tachyons of energies $\omega_{1}$ and $\omega_{2}$. As described in section 1 , this has the form:

$$
4 g_{s} \sinh \left(\pi\left|\omega_{1}\right|\right) \sinh \left(\pi\left|\omega_{2}\right|\right) f\left(\omega_{1}, \omega_{2}\right),
$$

where $f\left(\omega_{1}, \omega_{2}\right)$ is a sum of a finite piece and an apparently divergent piece:

$$
f\left(\omega_{1}, \omega_{2}\right)=f_{\text {finite }}\left(\omega_{1}, \omega_{2}\right)+f_{\text {div }}\left(\omega_{1}, \omega_{2}\right)
$$

$f_{\text {finite }}$ has been given in (1.8), but since we shall not need it, we do not reproduce it here. $f_{\text {div }}\left(\omega_{1}, \omega_{2}\right)$ is given by:

$$
f_{\text {div }}\left(\omega_{1}, \omega_{2}\right)=\frac{1}{2} \int_{0}^{1} d y y^{-2}\left(1+2 \omega_{1} \omega_{2} y\right)
$$

We shall follow the general strategy introduced in section 2 to evaluate $f_{\text {div }}$ (and also $g_{\text {div }}$ described in section 6). Let us suppose that we have some on-shell amplitude, expressed as an integration over the moduli $\vec{m} \equiv\left\{m_{1}, \cdots, m_{n}\right\}$ of the world-sheet with punctures. The issue that we have to deal with is that the integrand diverges at various corners of the moduli space and we have to make sense of these divergences. For this we express the amplitude as sum over Feynman diagrams of string field theory. Let us suppose that we have a Feynman diagram with $k$ propagators and that $q_{1}, \cdots q_{k}$ are the sewing parameters associated with these propagators, each taking value in the range $0 \leq q_{i} \leq 1$. The analysis of the type performed in section 4 determines the relation between the moduli $\vec{m}$ and the sewing parameters $q_{1}, \cdots, q_{k}$ and $(n-k)$ other variables $\left\{\tilde{m}_{1}, \cdots \tilde{m}_{n-k}\right\}$ arising from the interaction vertices of the Feynman diagram. We now express the integrand as a function of the $q_{i}$ 's and $\tilde{m}_{j}$ 's and expand it in a power series in $q_{i}$ 's, with the coefficients of the power series being functions of the $\tilde{m}_{j}$ 's. Next we apply the replacement rule (2.11) to extract a finite answer. We must also include, for each propagator, the contribution from the propagation of the $\psi^{1}$ field introduced in (2.14) separately. The effect of the Jacobian factors discussed in section 2.5 contribute at the same order as one loop amplitudes, and will affect the analysis of $g_{\text {div }}$ in section 6 . However they will not affect the analysis of $f_{\text {div }}$ that will be carried out in this section, since this, being a disk two point function, represents a tree amplitude.

To implement the strategy described above for the integral (5.3), we first need the world-sheet interpretation of $y$ appearing in (5.3). There is a simple answer to this: $i$ and $i y$ are the positions of the vertex operators carrying energies $\omega_{1}$ and $\omega_{2}$ in the upper half plane [4]. Therefore we can use the relation between $y$ and $q$ given in (4.16) for 
analyzing (5.3). We first divide the range of integration over $y$ into the regions $\mathrm{A}$ and $\mathrm{B}$ described in (4.17), (4.18). The contribution from the region B does not suffer from any divergences and simply gives back the same integral with integration range replaced by $\lambda^{-2} \leq y \leq 1$. The contribution from the region A is evaluated by replacing $y$ by $q / \lambda^{2}$ according to (4.16) and expressing the integral as

$$
\frac{1}{2} \int_{0}^{1} d q q^{-2}\left(\lambda^{2}+2 \omega_{1} \omega_{2} q\right) \quad \rightarrow \quad-\frac{1}{2} \lambda^{2},
$$

where in the second step we have used the replacement rule (2.11). Therefore the net result is

$$
\frac{1}{2} \int_{\lambda^{-2}}^{1} d y y^{-2}\left(1+2 \omega_{1} \omega_{2} y\right)-\frac{1}{2} \lambda^{2}=-\frac{1}{2}\left\{1-2 \omega_{1} \omega_{2} \ln \lambda^{2}\right\} .
$$

We also have to add the contribution where in figure 3(a) we replace the internal open string propagator by the $\psi^{1}$ propagator. This contribution however vanishes, since the open-closed string interaction vertex, with a closed string tachyon and an open string state associated with the vertex operator $\partial c$, vanishes for the choice of local coordinate system given in (4.3). To see this, note that if $c \bar{c} V_{T}$ denotes the closed string tachyon vertex operator, then this two point function is proportional to

$$
\left\langle\bar{c} c V_{T}(i) \partial c(0)\right\rangle \propto\left[\partial_{z}\{(z-i)(z+i)\}\right]_{z=0}=0 .
$$

Therefore (5.5) represents the full contribution to $f_{\text {div }}: 5$

$$
f_{\text {div }}\left(\omega_{1}, \omega_{2}\right)=-\frac{1}{2}\left(1-2 \omega_{1} \omega_{2} \ln \lambda^{2}\right) .
$$

Comparing this with (1.12) we get

$$
A_{f}=-\frac{1}{2}, \quad B_{f}=\ln \lambda^{2} .
$$

Note that the vanishing of the $\psi^{1}$ exchange amplitude is a specific property of the choice of local coordinate system that we have made in (4.3). If we had chosen a different coordinate system, then the result would not have vanished. However the final result for any physical amplitude will be independent of the choice of local coordinates. In particular $A_{f}$ will be independent of the choice of local coordinate system since it can be extracted from the scattering amplitude of $n$ closed string tachyons. We have illustrated this in appendix A by working with a different choice of local coordinate system for the C-O interaction vertex. $B_{f}$ by itself need not be independent of the choice of local coordinate system, but the combination $2 B_{g}-B_{f}$ will be independent of the choice of local coordinates.

\section{Annulus one point function in two dimensional string theory}

We now turn to the second building block for the amplitudes in two dimensional string theory - the one point function of closed string tachyon of energy $\omega$ on the annulus. As

\footnotetext{
${ }^{5}$ We shall follow the convention that $f_{\text {div }}$ stands for any contribution to the disk two point function that is not already included in $f_{\text {finite }}$ defined in (1.8). A similar convention will be followed for $g_{\text {div }}$ in section 6 .
} 
discussed in section 1 , this has the form:

$$
2 g_{s} \sinh (\pi|\omega|) g(\omega)
$$

where $g(\omega)$ is given by the sum of a finite piece and a divergent piece:

$$
g(\omega)=g_{\text {finite }}(\omega)+g_{\text {div }}(\omega) .
$$

The expression for $g_{\text {finite }}(\omega)$ has been given in (1.10) and will not be repeated here. $g_{\text {div }}(\omega)$ has the form:

$$
g_{\mathrm{div}}(\omega)=\int_{0}^{1} d v \int_{0}^{1 / 4} d x\left\{\frac{v^{-2}-v^{-1}}{\sin ^{2}(2 \pi x)}+2 \omega^{2} v^{-1}\right\} .
$$

As in section 5, we can analyze this by first relating the variables $v$ and $x$ to the parameters $\left\{q_{i}\right\}$ associated with the propagators of string field theory, and then using the replacement rule (2.11). We shall call this the world-sheet contribution to $g_{\text {div }}$. However there are several other contributions to the annulus one point function that are not captured by the world-sheet expression for $g_{\text {finite }}$. We shall include these also in the definition of $g_{\text {div }}$. This includes the contribution due to the $\psi^{1}$ field propagating in the internal lines of the Feynman diagrams of string field theory. We also have two possible field redefinitions whose associated Jacobians give additional contributions to the one point function of the closed string tachyon. The first one is associated with the redefinition that relates the zero mode in open string field theory to the collective coordinate associated with the translation of the D-instanton along the time direction. The second redefinition involves the ghost field $\psi^{2}$ introduced in (2.14). This can also be interpreted as the redefinition that relates the gauge transformation parameter in open string field theory associated with the vacuum state $|0\rangle$ to the rigid $\mathrm{U}(1)$ symmetry transformation parameter that gives a phase to any open string stretching from the D-instanton to another D-brane. We shall analyze each of these contributions in turn.

\subsection{World-sheet contribution}

We shall first analyze the contribution to (6.3) that we obtain by replacing the variables $x$ and/or $v$ near the singular points by appropriate variables $\left\{q_{i}\right\}$ associated with the propagators of string field theory, and then using the replacement rule (2.11). For this we need to know the world-sheet interpretation of the variables $v$ and $x$. As in (4.69), (4.70), $-i \ln v$ is the periodicity of the $w$ coordinate on the annulus, with the real part of $w$ taking value in the interval $(0, \pi)$, and $2 \pi x$ denotes the real part of the location of the closed string puncture in the $w$ plane [4]. We shall divide the integration region over $v$ and $x$ in (6.3) into four regions shown in figure 8, representing the contributions from different Feynman diagrams in figure 7, and carry out the integration over each region separately, using the replacement rule (2.11) or (3.4). In this analysis we shall ignore terms that vanish in the large $\alpha$ and large $\tilde{\lambda}$ limit, including terms proportional to $\alpha^{2} / \widetilde{\lambda}$ and $\widetilde{\lambda}^{2} / \alpha^{2}$, since these terms must cancel at the end. The reader may be somewhat uncomfortable in ignoring terms of order $\widetilde{\lambda}^{2} / \alpha^{2}$, since this ratio, being given by $\lambda^{2}$, is large. Nevertheless, it follows 
from our general arguments that such terms must cancel at the end. As discussed at the end of this subsection, we have explicitly checked that such terms do cancel.

We begin with the contribution from the region (d) given in (4.102):

$$
\begin{aligned}
& \frac{\pi}{2} \geq 2 \pi x \geq \widetilde{\lambda}^{-1}\left(1-\alpha^{-2}\right), \\
& \frac{1}{\alpha^{2} \widetilde{\lambda}^{2} \sin ^{2}(2 \pi x)}\left(1+\frac{1}{4 \widetilde{\lambda}^{2}}\right)^{-2}\left[1-2\left\{\cot ^{2}(2 \pi x)-\widetilde{\lambda}^{2} f^{2}\right\} \alpha^{-2} \widetilde{\lambda}^{-2}\left(1+\frac{1}{4 \widetilde{\lambda}^{2}}\right)^{-2}\right] \leq v<1
\end{aligned}
$$

where $f$ stands for $f(\tan (\pi x))$ The integral takes the form:

$$
\begin{aligned}
I_{(d)}= & \int_{(2 \pi \widetilde{\lambda})^{-1}\left(1-\alpha^{-2}\right)}^{1 / 4} d x \int_{\frac{1}{\alpha^{2} \widetilde{\lambda}^{2} \sin ^{2}(2 \pi x)}\left(1+\frac{1}{4 \widetilde{\lambda}^{2}}\right)^{-2}\left[1-2\left\{\cot ^{2}(2 \pi x)-\widetilde{\lambda}^{2} f^{2}\right\} \alpha^{-2} \widetilde{\lambda}^{-2}\left(1+\frac{1}{4 \widetilde{\lambda}^{2}}\right)^{-2}\right] d v}^{1} \\
& \times\left[\frac{1}{\sin ^{2}(2 \pi x)}\left(v^{-2}-v^{-1}\right)+2 \omega^{2} v^{-1}\right] \\
= & \int_{(2 \pi \widetilde{\lambda})^{-1}\left(1-\alpha^{-2}\right)}^{1 / 4} d x\left[\alpha^{2} \widetilde{\lambda}^{2}+\frac{\alpha^{2}}{2}+2\left\{\cot ^{2}(2 \pi x)-\widetilde{\lambda}^{2} f^{2}\right\}-\frac{1}{\sin ^{2}(2 \pi x)}\right. \\
& \left.-\frac{1}{\sin ^{2}(2 \pi x)} \ln \left\{\alpha^{2} \widetilde{\lambda}^{2} \sin ^{2}(2 \pi x)\right\}+2 \omega^{2} \ln \left\{\alpha^{2} \widetilde{\lambda}^{2} \sin ^{2}(2 \pi x)\right\}\right] .
\end{aligned}
$$

The integrals in the third line on the right hand side are elementary. For the terms in the last line, the integral can be simplified by writing $d x / \sin ^{2}(2 \pi x)$ as $-d \cot (2 \pi x) / 2 \pi$ and then doing an integration by parts. This gives, after dropping terms that have either powers of $\widetilde{\lambda}$ or of $\alpha$ in the denominator:

$$
\begin{aligned}
I_{(d)}= & {\left[\frac{1}{4} \alpha^{2} \widetilde{\lambda}^{2}-\frac{\alpha^{2} \widetilde{\lambda}}{2 \pi}+\frac{\widetilde{\lambda}}{2 \pi}+\frac{\alpha^{2}}{8}+\frac{\widetilde{\lambda}}{\pi}-\frac{1}{2}-2 \widetilde{\lambda}^{2} \int_{(2 \pi \widetilde{\lambda})^{-1}}^{\frac{1}{4}} d x f(\tan (\pi x))^{2}-\frac{\tilde{\lambda}}{2 \pi}\right.} \\
& \left.+\frac{1}{2 \pi}\{-2 \widetilde{\lambda} \ln \alpha+\pi-2 \widetilde{\lambda}\}+\frac{1}{2} \omega^{2} \ln \frac{\alpha^{2} \widetilde{\lambda}^{2}}{4}\right] .
\end{aligned}
$$

An alternative procedure for evaluating the $\omega$ independent part of the integral is to express the integrand as a total derivative, e.g. as,

$$
d \mathcal{A}, \quad \mathcal{A}=-v^{-1} \frac{1}{\sin ^{2}(2 \pi x)} d x-\frac{1}{2 \pi} v^{-1} \cot (2 \pi x) d v,
$$

and integrate the one form $\mathcal{A}$ along the boundary of region (d) in the anti-clockwise direction. This has the advantage that we do not need to explicitly find the shape of the boundary given in (4.102). For example, while evaluating the contribution from the boundary separating regions (c) and (d), we can simply change variables from $(v, x)$ to $(u, \beta)$ using (4.98) that is appropriate in region (c), set $u=\alpha^{-2}\left(4 \widetilde{\lambda}^{2}\right)^{2}\left(1+4 \widetilde{\lambda}^{2}\right)^{-2}$ to parametrize the boundary, and then integrate over the variable $\beta$ in the range $(2 \widetilde{\lambda})^{-1} \leq \beta \leq 1$. This give the same result, but this procedure is easier to use if we want to extend the analysis to higher orders in the expansion in powers of $\widetilde{\lambda}^{-1}$ and $\alpha^{-2}$ and check that these terms indeed cancel, as implied by our general arguments. 
Next we turn to the contribution from region (c). In order to evaluate the contribution from this region, we need to change variables from $(v, x)$ to $(u, \beta)$ according to (4.98),

$$
\begin{aligned}
2 \pi x & =2 \tan ^{-1} \beta+2 u f \widetilde{\lambda}^{-1}-\frac{1}{\beta}\left(1-\beta^{2}\right) \tilde{\lambda}^{-2} u, \\
v & =u \frac{\left(1+\beta^{2}\right)^{2}}{4 \beta^{2} \widetilde{\lambda}^{2}}\left\{1+u \widetilde{\lambda}^{-2} \frac{1}{2 \beta^{2}}\left(1-\beta^{2}-2 \beta \widetilde{\lambda} f\right)^{2}\right\},
\end{aligned}
$$

and then integrate over the range $0 \leq u \leq \alpha^{-2}\left\{1+\left(4 \widetilde{\lambda}^{2}\right)^{-1}\right\}^{-2}$ and $(2 \widetilde{\lambda})^{-1} \leq \beta \leq 1$ using the replacement rule (3.4). One finds:

$$
\begin{aligned}
I_{(c)}=\frac{1}{\pi} \int_{1 /(2 \widetilde{\lambda})}^{1} d \beta \int_{0}^{\alpha^{-2}\left(1+\left(4 \widetilde{\lambda}^{2}\right)^{-1}\right)^{-2}} d u[ & \widetilde{\lambda}^{2} u^{-2} \frac{1}{1+\beta^{2}}+\widetilde{\lambda} u^{-1} f^{\prime}(\beta)+\frac{1}{4} u^{-1} \frac{1+\beta^{2}}{\beta^{2}} \\
& \left.+\frac{2}{1+\beta^{2}} \omega^{2} u^{-1}\right] \\
\rightarrow- & -\frac{1}{\pi} \alpha^{2} \widetilde{\lambda}^{2}\left(1+\frac{1}{4 \widetilde{\lambda}^{2}}\right)^{2} \int_{1 /(2 \widetilde{\lambda})}^{1} \frac{d \beta}{1+\beta^{2}}=\left[-\frac{1}{4} \alpha^{2} \widetilde{\lambda}^{2}-\frac{\alpha^{2}}{8}+\frac{\alpha^{2} \widetilde{\lambda}}{2 \pi}\right]
\end{aligned}
$$

In region (b), $v$ is restricted to the range $\left(\alpha^{2}-\frac{1}{2}\right)^{-1} \leq v \leq 1$, and we use (4.87) to trade in $x$ for the variable $u$ via

$$
2 \pi x=u\left(1-\alpha^{-2}\right), \quad 0 \leq u \leq \tilde{\lambda}^{-1} .
$$

This reduces the integral to

$$
\begin{aligned}
I_{(b)} & =\int_{\left(\alpha^{2}-\frac{1}{2}\right)^{-1}}^{1} d v \int_{0}^{\tilde{\lambda}^{-1}} \frac{d u}{2 \pi}\left[\left(1+\alpha^{-2}\right)\left(v^{-2}-v^{-1}\right)\left\{u^{-2}+\mathcal{O}(1)\right\}+\left(1-\alpha^{-2}\right) 2 \omega^{2} v^{-1}\right] \\
& \rightarrow \frac{1}{2 \pi}\left[-\tilde{\lambda}\left(1+\alpha^{-2}\right) \int_{\left(\alpha^{2}-\frac{1}{2}\right)^{-1}}^{1} d v\left(v^{-2}-v^{-1}\right)\right]=\left[-\frac{1}{2 \pi} \tilde{\lambda} \alpha^{2}+\frac{\tilde{\lambda}}{4 \pi}+\frac{\tilde{\lambda}}{\pi} \ln \alpha\right] .
\end{aligned}
$$

Finally in region (a) the relevant change of variables is given in (4.81):

$$
v=u_{2}\left\{1+\frac{1}{2} u_{2}\right\}, \quad x=\frac{1}{2 \pi} u_{1}\left(1-u_{2}\right), \quad 0 \leq u_{1} \leq \tilde{\lambda}^{-1}, \quad 0 \leq u_{2} \leq \alpha^{-2},
$$

ignoring fractional errors of order $u_{1}^{2}$ or $u_{2}^{2}$. This gives

$$
d x \wedge d v=\frac{1}{2 \pi} d u_{1} \wedge d u_{2},
$$

and expresses the contribution to the integral from this region as

$$
\begin{aligned}
I_{(a)}=\frac{1}{2 \pi} \int_{0}^{\widetilde{\lambda}^{-1}} d u_{1} \int_{0}^{\alpha^{-2}} d u_{2} & {\left[u_{1}^{-2}\left(1+2 u_{2}\right)\left\{u_{2}^{-2}\left(1-u_{2}\right)-u_{2}^{-1}\left(1-\frac{1}{2} u_{2}\right)\right\}\right.} \\
& \left.+2 \omega^{2} u_{2}^{-1}\left(1-\frac{1}{2} u_{2}\right)\right] \\
=\frac{1}{2 \pi} \int_{0}^{\widetilde{\lambda}^{-1}} d u_{1} \int_{0}^{\alpha^{-2}} d u_{2} & u_{1}^{-2}\left\{u_{2}^{-2}\left(1+u_{2}\right)-u_{2}^{-1}\left(1+\frac{3}{2} u_{2}\right)+2 \omega^{2} u_{2}^{-1}\left(1-\frac{1}{2} u_{2}\right)\right\} .
\end{aligned}
$$


We now use the replacement rule (3.4) to get:

$$
I_{(a)}=\frac{\tilde{\lambda} \alpha^{2}}{2 \pi} .
$$

Adding (6.6), (6.9), (6.11) and (6.15), we get the total world-sheet contribution to $g_{\text {div }}$ :

$$
\begin{aligned}
g_{\text {world }} & =\left[-2 \widetilde{\lambda}^{2} \int_{(2 \pi \widetilde{\lambda})^{-1}}^{\frac{1}{4}} d x f(\tan (\pi x))^{2}+\frac{\widetilde{\lambda}}{4 \pi}+\frac{1}{2} \omega^{2} \ln \frac{\alpha^{2} \widetilde{\lambda}^{2}}{4}\right] \\
& =\left[-\frac{2}{\pi} \widetilde{\lambda}^{2} \int_{(2 \widetilde{\lambda})^{-1}}^{1} d \beta\left(1+\beta^{2}\right)^{-1} f(\beta)^{2}+\frac{\widetilde{\lambda}}{4 \pi}+\frac{1}{2} \omega^{2} \ln \frac{\alpha^{2} \widetilde{\lambda}^{2}}{4}\right],
\end{aligned}
$$

where in the last step we have changed variable from $x$ to $\beta=\tan (\pi x)$ and dropped terms containing inverse powers of $\tilde{\lambda}$.

We have carried out two further checks on the result:

1. Terms of order $\widetilde{\lambda}^{2} / \alpha^{2}$ have been dropped in our analysis, despite the ratio being large, since we have argued that such terms must cancel at the end. To confirm this, we have checked that such terms appear in the expressions for $I_{(c)}$ and $I_{(d)}$, and are given respectively by,

$$
-\frac{1}{\pi} \frac{\tilde{\lambda}^{2}}{\alpha^{2}} \int_{(2 \widetilde{\lambda})^{-1}}^{1} \frac{d \beta}{\left(1+\beta^{2}\right)} f(\beta)^{4}
$$

and

$$
\frac{1}{\pi} \frac{\widetilde{\lambda}^{2}}{\alpha^{2}} \int_{(2 \widetilde{\lambda})^{-1}}^{1} \frac{d \beta}{\left(1+\beta^{2}\right)} f(\beta)^{4} .
$$

This shows that these terms do cancel at the end.

2. The $\omega$ independent part of the integral in (6.3) can also be computed using the alternative procedure described in section 2.4. For this we note that this part of the integrand may be expressed as a total derivative, e.g. as,

$$
d \mathcal{A}, \quad \mathcal{A}=-v^{-1} \frac{1}{\sin ^{2}(2 \pi x)} d x-\frac{1}{2 \pi} v^{-1} \cot (2 \pi x) d v .
$$

Therefore the integral can be expressed as an anti-clockwise boundary integral of $\mathcal{A}$ in figure 8 , with the boundaries containing the lines $v=1$ and $x=1 / 4$, and the regularized boundaries at $v=0$ and $x=0$. The latter two may be viewed as the collection of boundaries at $q_{2}=\delta_{2}$ in figures $7(\mathrm{a})$ and (c) and at $q_{1}=\delta_{1}$ in figures 7(a) and (b). We have checked that this gives the same result as (6.16) after dropping all terms proportional to $\delta_{1}^{-1}, \delta_{2}^{-1}, \ln \delta_{1}$ and $\ln \delta_{2}$ in the final expression, as described in section 2.4.

\section{$6.2 \psi^{1}$ exchange contribution}

We shall now evaluate the $\psi^{1}$ exchange contributions. For this we need the $\psi^{1}$ propagator. In order to demonstrate that the final results are independent of the choice of normalization conventions, we shall normalize the vacuum as

$$
\left\langle 0\left|c_{-1} c_{0} c_{1}\right| 0\right\rangle=K_{0}
$$




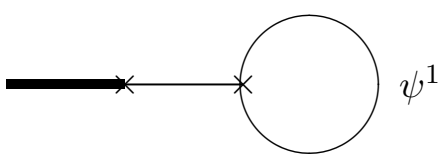

(a)

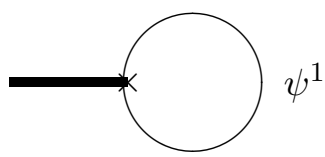

(b)

Figure 9. Diagrams involving $\psi^{1}$ propagator that contribute to the annulus one point function of the closed string tachyon.

where $K_{0}$ is some non-zero constant. This corresponds to normalizing the ghost correlation function on the upper half plane as:

$$
\left\langle c\left(z_{1}\right) c\left(z_{2}\right) c\left(z_{3}\right)\right\rangle=K_{0}\left(z_{1}-z_{2}\right)\left(z_{2}-z_{3}\right)\left(z_{1}-z_{3}\right) .
$$

If we expand the string field as

$$
|\Psi\rangle=\psi^{0} c_{1}|0\rangle+\psi^{1} c_{0}|0\rangle+\cdots
$$

then with the convention (6.20) the quadratic term in the string field theory action takes the form:

$$
\frac{1}{2}\left\langle\Psi\left|Q_{B}\right| \Psi\right\rangle=-\frac{K_{0}}{2}\left(\psi^{0}\right)^{2}-K_{0}\left(\psi^{1}\right)^{2} .
$$

Therefore the tachyon propagator is given by $K_{0}^{-1}$ while the $\psi^{1}$ propagator is given by $K_{0}^{-1} / 2$. Although the propagators are convention dependent, their ratio is convention independent. Our final results will depend only on the convention independent ratios of this type.

The C-O amplitude, where the closed string is an on-shell tachyon state and the open string represents $\psi^{1}$, vanishes by (5.6). Therefore the relevant diagrams are the ones shown in figure 9 . Both these diagrams involve computing a disk amplitude with one external $\mathrm{C}$ and two external O's, with the O's corresponding to the $\psi^{1}$ state, and then multiplying it by the $\psi^{1}$ propagator. One subtlety in this computation is that the $\psi^{1}$ vertex operator $\partial c$ is not a dimension zero primary of the form $c W$ where $W$ is a dimension one matter primary. Therefore we need to be careful in evaluating the integrand that we shall eventually integrate over the modulus of this diagram - the variable $q$ appearing in (4.9) for figure 9 (a) and the variable $\beta$ appearing in (4.12) for figure 9 (b). Defining $\beta=q /(2 \widetilde{\lambda})$ in (4.9), we can analyze the two diagrams together. Let $z$ be the coordinate on the upper half plane in which the closed string puncture is situated at $i$ and the open string punctures are situated at $z_{1}=-\beta$ and $z_{2}=\beta$. Let $w_{1}$ and $w_{2}$ be the local coordinates at the open string punctures, related to $z$ via the relations

$$
z=F_{a}\left(w_{a}, \beta\right)
$$

so that $z_{a}=F_{a}(0, \beta)$. Then the integrand multiplying $d \beta$ is given by the correlation function of the vertex operators of the closed string tachyon at $i$ and of the two $\psi^{1}$ 's at $z_{1}$, 
$z_{2}$ in the $w_{1}, w_{2}$ coordinate system, and the operator $[11,20,21]$

$$
-K_{1} \sum_{a=1}^{2} \oint_{a} \frac{\partial F_{a}}{\partial \beta} b(z) d z
$$

where $\oint_{a}$ is the anti-clockwise contour around $z_{a}=F_{a}(0 ; \beta)$ and $K_{1}$ is another normalization constant. Note that $\partial F_{a} / \partial \beta$ is computed at fixed $w_{a}$, but after taking the derivative we need to express this back in the $z$ coordinate system using (6.24). Therefore the amplitude takes the form:

$$
-K_{1} \int d \beta \sum_{a=1}^{2} \oint_{a} \frac{\partial F_{a}}{\partial \beta} d z\left\langle b(z) F_{1} \circ \partial c(0) F_{2} \circ \partial c(0) c \bar{c} V_{T}(i)\right\rangle,
$$

where $c \bar{c} V_{T}$ denotes the vertex operator of the closed string tachyon of energy $\omega$ and $F_{a} \circ \partial c(0)$ is the conformal transform of $\partial c(0)$ by the map $F_{a}$. After computing the amplitude we can divide the result by $2 g_{s} \sinh (\pi|\omega|)$ to compute its contribution to $g(\omega)$. We describe this computation in detail in appendix B, quoting here only the final results for the contribution $g_{\psi^{1}}$ to $g_{\text {div }}$ :

$$
g_{\psi^{1}}(\omega)=\left[\frac{2}{\pi} \widetilde{\lambda}^{2} \int_{(2 \widetilde{\lambda})^{-1}}^{1} d \beta\left(1+\beta^{2}\right)^{-1} f(\beta)^{2}+\frac{\widetilde{\lambda}}{4 \pi}\right] .
$$

The first term inside the square bracket is the contribution from figure $9(\mathrm{~b})$ and the second term is the contribution from figure 9 (a). The constants $K_{0}$ and $K_{1}$ drop out of the final computation since we compare the result with the contributions where the $\psi^{1}$ in figure 9 is replaced by the open string tachyon. The latter contributions can be read out from (6.14) and (6.9) respectively.

\subsection{C-C-O disk amplitude, zero mode field redefinition and the Jacobian}

In this subsection we shall explore whether we need a field redefinition to relate the zero mode field $\phi$ of the open string field theory associated with the vertex operator $c \partial X$ and the collective coordinate $\tilde{\phi}$ that translates the D-instanton along the time direction. This will be done by studying the C-C-O disk amplitude with a pair of closed string tachyon vertex operators carrying total energy $\omega$ and the open string vertex operator $c \partial X$. If the open string zero mode had been the same as the collective coordinate, then this amplitude would be given by $-i \omega$ times the $\mathrm{C}-\mathrm{C}$ disk amplitude, since the dependence of the amplitude on the collective coordinate $\widetilde{\phi}$ is expected to be of the form $e^{-i \omega \widetilde{\phi}}$. Therefore any deviation from this form would have to be removed by a field redefinition and the associated Jacobian would have to be taken into account in computing amplitudes.

Therefore we consider the C-C-O amplitude, with the closed string states corresponding to a pair of tachyons as in section 5 and the open string state corresponding to the zero mode $\phi$ associated with the vertex operator $c \partial X$. As in the previous cases, here by amplitude we shall refer to the building block, obtained before getting the energy conserving delta function or the $\mathcal{N} e^{-1 / g_{s}}$ factor. We shall insert the closed string vertex operators at $i$ and 
$i y$ as in section 5 , and the open string vertex operator at a point $x$ on the real axis, and express the amplitude as an integral over $x$ and $y$. The integrand is given by the integrand of the C-C amplitude, multiplied by ${ }^{6}$

$$
\frac{1}{2 \pi}\left[-\frac{\omega_{1}}{x-i}+\frac{\omega_{1}}{x+i}-\frac{\omega_{2}}{x-i y}+\frac{\omega_{2}}{x+i y}\right]=-\frac{i}{\pi}\left[\frac{\omega_{1}}{1+x^{2}}+\frac{\omega_{2} y}{x^{2}+y^{2}}\right] .
$$

Therefore if we integrate over $x$ for fixed $y$, we get the result:

$$
-i\left(\omega_{1}+\omega_{2}\right)
$$

multiplying the integrand of the C-C amplitude. Since the coupling of the collective coordinate $\widetilde{\phi}$ to this order is also supposed to be given by $-i\left(\omega_{1}+\omega_{2}\right)$ times the C-C amplitude, this would be consistent with the hypothesis that $\phi$ and $\widetilde{\phi}$ can be identified.

This analysis, however is only formal, since the C-C-O amplitude has divergences from different regions of integration and they need to be treated following the replacement rule (2.11). Therefore we need to carefully evaluate the contributions from the different regions shown in figure 5, associated with the Feynman diagrams of figure 4. Possible divergences are associated with the $q$ parameters of one or more internal propagators in figure 4 approaching zero. To analyze this, we shall split the integrand into the sum of two parts - one where we multiply (6.28) by the integrand of $f_{\text {finite }}$ defined in (1.8), and the other where we multiply (6.28) by the integrand of $f_{\text {div }}$ defined in (1.9). Now from figure 5 one can easily see that all the potentially divergent regions I, I', II, II', III and III', associated with Feynman diagrams with at least one propagator, correspond to the small $y$ region. On the other hand, $f_{\text {finite }}$ defined in (1.8) has the property that it has no divergence from the small $y$ region. We also see from (6.28) that integration over $x$ for fixed $y$ does not produce any additional singularity as $y \rightarrow 0$. Therefore if we multiply the integrand of $f_{\text {finite }}$ by (6.28) and carry out the integration over $x$ and $y$, we can exclude the region $y \leq \epsilon$ from the $y$ integral at the cost of making an error that will be suppressed by a power of $\epsilon$ and will vanish in the $\epsilon \rightarrow 0$ limit. On the other hand, by the argument outlined in the previous paragraph, the contribution to the $x$ integral for $y \geq \epsilon$ will just produce a multiplicative factor of $-i\left(\omega_{1}+\omega_{2}\right)$, reproducing the expected coupling of the collective coordinate. Therefore the problematic terms come from the second integral where we multiply the integrand of $f_{\text {div }}$ by (6.28) and integrate over $x$ and $y$. This contribution to the $\mathrm{C}-\mathrm{C}-\mathrm{O}$ amplitude is given by:

$$
\begin{aligned}
\int_{-\infty}^{\infty} d x \int_{0}^{1} d y \frac{1}{2 \pi} & {\left[-\frac{\omega_{1}}{x-i}+\frac{\omega_{1}}{x+i}-\frac{\omega_{2}}{x-i y}+\frac{\omega_{2}}{x+i y}\right] } \\
& \times 4 g_{s} \sinh \left(\pi\left|\omega_{1}\right|\right) \sinh \left(\pi\left|\omega_{2}\right|\right)\left[\frac{1}{2} y^{-2}\left(1+2 \omega_{1} \omega_{2} y\right)\right] .
\end{aligned}
$$

A reader unconvinced by this argument can verify this explicitly by replacing the second line of (6.30) by $y^{\beta}$ for $\beta \geq 0$, and then carrying out the integration over $x$ and $y$ from

\footnotetext{
${ }^{6}$ The overall normalization has been adjusted to be $1 / 2 \pi$ by choosing suitable normalization of the open string zero mode $\phi$.
} 
different regions of figure 5 following the same procedure as described in appendix C. For $\beta \geq 0$, the result will always be given by $-i\left(\omega_{1}+\omega_{2}\right)$ times the integral of $y^{\beta}$.

In appendix $\mathrm{C}$ we have analyzed the contributions to (6.30) from the different regions shown in figure 5, associated with the Feynman diagrams of figure 4 . The result is:

$$
2 i\left(\omega_{1}+\omega_{2}\right) g_{s} \sinh \left(\pi\left|\omega_{1}\right|\right) \sinh \left(\pi\left|\omega_{2}\right|\right)\left\{1-\frac{2}{\pi} \tilde{\lambda}-2 \omega_{1} \omega_{2} \ln \widetilde{\lambda}^{2}\right\} .
$$

The desired result for this is $-i\left(\omega_{1}+\omega_{2}\right)$ times the divergent part of the C-C amplitude given in (5.7):

$$
i\left(\omega_{1}+\omega_{2}\right) \times 4 g_{s} \sinh \left(\pi\left|\omega_{1}\right|\right) \sinh \left(\pi\left|\omega_{2}\right|\right) \frac{1}{2}\left\{1-2 \omega_{1} \omega_{2} \ln \lambda^{2}\right\} .
$$

Note that we have put back the overall normalization factor $4 g_{s} \sinh \left(\pi\left|\omega_{1}\right|\right) \sinh \left(\pi\left|\omega_{2}\right|\right)$ that multiplies $f\left(\omega_{1}, \omega_{2}\right)$ in the disk two point amplitude. We now see that (6.31) differs from (6.32) by the term:

$$
-2 i\left(\omega_{1}+\omega_{2}\right) g_{s} \sinh \left(\pi\left|\omega_{1}\right|\right) \sinh \left(\pi\left|\omega_{2}\right|\right)\left\{\frac{2}{\pi} \tilde{\lambda}+2 \omega_{1} \omega_{2} \ln \frac{\widetilde{\lambda}^{2}}{\lambda^{2}}\right\} .
$$

This means that the zero mode dependence of the effective action is not of the form $e^{-i\left(\omega_{1}+\omega_{2}\right) \phi}$ times the amplitude without the zero mode. Let us denote by $\Phi_{C}(\omega, P)$ the closed string tachyon field of energy $\omega$ and Liouville momentum $P$, with positive $\omega$ describing outgoing states and negative $\omega$ describing incoming states. Then the extra term in the effective action to order $\phi$ is of the form ${ }^{7}$

$$
\begin{aligned}
S_{\text {extra }}= & -i g_{s} \phi \int d \omega_{1} d P_{1} d \omega_{2} d P_{2}\left(\omega_{1}+\omega_{2}\right) \sinh \left(\pi\left|\omega_{1}\right|\right) \sinh \left(\pi\left|\omega_{2}\right|\right)\left\{\frac{2}{\pi} \widetilde{\lambda}+2 \omega_{1} \omega_{2} \ln \frac{\widetilde{\lambda}^{2}}{\lambda^{2}}\right\} \\
& \times \Phi_{C}\left(\omega_{1}, P_{1}\right) \Phi_{C}\left(\omega_{2}, P_{2}\right) .
\end{aligned}
$$

Note that the integrand could be multiplied by some function of $\left\{\omega_{i}\right\}$ and $\left\{P_{i}\right\}$ that reduces to 1 for on-shell closed string fields. But since eventually we shall use this to compute onshell amplitudes, this factor will not be important and we shall drop it. We shall now look for an appropriate relation between the open string field $\phi$ and the collective coordinate $\widetilde{\phi}$ so that the effective action has $\tilde{\phi}$ dependence proportional to $e^{i \omega \widetilde{\phi}}$. This will allow us to recover the standard energy conserving delta function after integration over $\tilde{\phi}$.

For closed string tachyon of energy $\omega$, the one point function on the disk is given by,

$$
2 \sinh (\pi|\omega|) .
$$

The C-O amplitude with a closed string tachyon and an open string zero mode $\phi$ will be given by $-i \omega$ times this amplitude. This shows the presence of a term in the effective action of the form

$$
-2 i \phi \int d \omega d P \omega \sinh (\pi|\omega|) \Phi_{C}(\omega, P)
$$

\footnotetext{
${ }^{7}$ We are using the convention that if $S$ is the action then the weight factor in the path integral is $e^{S}$. In that case a term in the action contributes to the amplitude without any additional minus sign or factors of $i$. Also note that when we compute the amplitude from (6.34), we shall get an extra factor of 2 due to two possible ways of contracting $\Phi_{C}$ with the external closed string states. This would account for the factor of 2 in (6.33).
} 
Since there is no divergence in this amplitude, this result is not modified. We now make a field redefinition

$$
\phi=\widetilde{\phi}+2 g_{s} \widetilde{\phi} \int d \omega^{\prime} d P^{\prime} \sinh \left(\pi\left|\omega^{\prime}\right|\right) a\left(\omega^{\prime}\right) \Phi_{C}\left(\omega^{\prime}, P^{\prime}\right),
$$

for some function $a(\omega)$ that will be determined shortly. Upon substitution into (6.36), this produces $(6.36)$ with $\phi$ replaced by $\widetilde{\phi}$, plus an extra term:

$$
\begin{aligned}
& -4 i g_{s} \widetilde{\phi} \int d \omega d P \int d \omega^{\prime} d P^{\prime} \omega \sinh (\pi|\omega|) \Phi_{C}(\omega, P) \sinh \left(\pi\left|\omega^{\prime}\right|\right) a\left(\omega^{\prime}\right) \Phi_{C}\left(\omega^{\prime}, P^{\prime}\right) \\
& =-2 i g_{s} \widetilde{\phi} \int d \omega d P \int d \omega^{\prime} d P^{\prime} \sinh (\pi|\omega|) \sinh \left(\pi\left|\omega^{\prime}\right|\right)\left\{\omega a\left(\omega^{\prime}\right)+\omega^{\prime} a(\omega)\right\} \\
& \quad \times \Phi_{C}(\omega, P) \Phi_{C}\left(\omega^{\prime}, P^{\prime}\right)
\end{aligned}
$$

where in the second step we have symmetrized the integrand in $\omega$ and $\omega^{\prime}$. On the other hand, to order $g_{s},(6.34)$ retains the same form with $\phi$ replaced by $\widetilde{\phi}$. If we choose

$$
a(\omega)=-\left[\frac{1}{\pi} \tilde{\lambda}+\omega^{2} \ln \frac{\widetilde{\lambda}^{2}}{\lambda^{2}}\right],
$$

then (6.38) cancels the unwanted extra term (6.34) in the effective action. Therefore we can identify $\widetilde{\phi}$ as the collective coordinate to this order.

When we express the path integral over $\phi$ in terms of path integral over $\widetilde{\phi}$ using the field redefinition (6.37), we get a Jacobian

$$
\begin{aligned}
1+ & 2 g_{s} \int d \omega^{\prime} d P^{\prime} \sinh \left(\pi\left|\omega^{\prime}\right|\right) a\left(\omega^{\prime}\right) \Phi_{C}\left(\omega^{\prime}, P^{\prime}\right) \\
& =\exp \left[2 g_{s} \int d \omega^{\prime} d P^{\prime} \sinh \left(\pi\left|\omega^{\prime}\right|\right) a\left(\omega^{\prime}\right) \Phi_{C}\left(\omega^{\prime}, P^{\prime}\right)+\mathcal{O}\left(g_{s}^{2}\right)\right] .
\end{aligned}
$$

This can be interpreted as a new term in the effective action, given by:

$$
\begin{aligned}
2 g_{s} & \int d \omega^{\prime} d P^{\prime} \sinh \left(\pi\left|\omega^{\prime}\right|\right) a\left(\omega^{\prime}\right) \Phi_{C}\left(\omega^{\prime}, P^{\prime}\right) \\
& =-2 g_{s} \int d \omega d P \sinh (\pi|\omega|)\left[\frac{1}{\pi} \tilde{\lambda}+\omega^{2} \ln \frac{\widetilde{\lambda}^{2}}{\lambda^{2}}\right] \Phi_{C}(\omega, P)
\end{aligned}
$$

This will give an additional contribution to the one point function of closed string tachyon besides the ones given by Feynman diagrams:

$$
-2 g_{s} \sinh (\pi|\omega|)\left[\frac{1}{\pi} \widetilde{\lambda}+\omega^{2} \ln \frac{\widetilde{\lambda}^{2}}{\lambda^{2}}\right] .
$$

Comparing this with (6.1) we see that (6.42) can be interpreted as an additional contribution to $g(\omega)$, given by:

$$
g_{\mathrm{jac}}(\omega)=-\left[\frac{1}{\pi} \tilde{\lambda}+\omega^{2} \ln \frac{\widetilde{\lambda}^{2}}{\lambda^{2}}\right] .
$$

We end this section with two remarks: 
1. The field redefinition described here is only one of the many field redefinitions that $\phi$ undergoes. In general (6.37) will contain terms involving other closed string fields and higher powers of closed string fields. These will affect higher order results but will not affect the closed string tachyon one point function to the order that we are doing our computation. There will also be terms that involve redefinition of $\phi$ involving higher powers of $\phi$ [13]. Such terms will lead to Jacobian factor involving non-linear terms in $\widetilde{\phi}$, which can be regarded as a contribution to the effective action for $\widetilde{\phi}$. However all such terms must cancel with explicit loop contribution to the effective action for $\phi$, so that when the dependence of the amplitude on $\widetilde{\phi}$ takes the form $e^{-i \omega \widetilde{\phi}}$, the integration measure becomes $\widetilde{\phi}$ independent. In this case the integration over $\tilde{\phi}$ will produce the $\delta(\omega)$ factor.

2. The notion of effective action used here is different from the notion of closed string effective action discussed in [41]. Here the effective action refers to the sum over contributions from connected world-sheet before integrating over the collective modes, while in the analysis of [41] the effective action includes disconnected world-sheets and also include the final integration over the collective modes. Therefore in the analysis of [41] the Jacobian given in (6.40) will appear as a multiplicative contribution to the integrand that, after integration over the collective coordinates, gives the effective action. The difference between the two papers can be traced to the fact that here we are trying to analyze the building blocks of the amplitude instead of the full amplitude.

\subsection{C-O-O-O disk amplitude, ghost field redefinition and the Jacobian}

In this subsection we shall find the relation between the infinitesimal gauge transformation parameter $\theta$ corresponding to the state $|0\rangle$ in open string field theory and the rigid $\mathrm{U}(1)$ transformation parameter $\widetilde{\theta}$. This will be done as follows. Let us imagine bringing a second D-instanton, which we shall call the spectator, on top of the original D-instanton, and consider an open string that stretches from the original D-instanton to the spectator D-instanton. We shall compare the transformation laws of this state under the gauge transformation of string field theory with the expected transformation laws under rigid $\mathrm{U}(1)$ to find the relation between the two gauge transformation parameters. For definiteness we shall work with a particular field $\xi$ that multiplies the vacuum state $|0\rangle$ of the open string stretched between the two D-instantons. The same analysis may be carried out with any other physical state of the open string stretched between the two D-instantons e.g. the field described by the vertex operator $c \partial X$. We have verified that this gives the same relation between $\theta$ and $\widetilde{\theta}$.

The conjugate anti-field $\xi^{*}$ of $\xi$ will have vertex operator $c \partial c \partial^{2} c / 2$ and will stretch from the spectator D-instanton to the original D-instanton. The states associated with the fields $\xi$ and $\xi^{*}$ may be assigned Chan-Paton factors $\left(\begin{array}{ll}0 & 1 \\ 0 & 0\end{array}\right)$ and $\left(\begin{array}{ll}0 & 0 \\ 1 & 0\end{array}\right)$ respectively, while the open string states with both ends lying on the original D-instanton will have ChanPaton factor $\left(\begin{array}{ll}1 & 0 \\ 0 & 0\end{array}\right)$. Now from the gauge transformation laws of the string fields discussed 
below (2.6), it follows that the gauge transformation of $\xi$ under the gauge transformation generated by $\theta$ is given by the second derivative of the Wilsonian effective action with respect to $\xi^{*}$ and $\psi^{2}$, where $\psi^{2}$ is the string field multiplying $|0\rangle \otimes\left(\begin{array}{ll}1 & 0 \\ 0 & 0\end{array}\right)$. The lowest order contribution to this gauge transformation law comes from the $\xi-\xi^{*}-\psi^{2}$ coupling in the action, whose coefficient is given by:

$$
\left\langle I\left(z_{1}\right)\left\{c \partial c \partial^{2} c\left(z_{2}\right) / 2\right\} I\left(z_{3}\right)\right\rangle \operatorname{Tr}\left[\left(\begin{array}{ll}
0 & 1 \\
0 & 0
\end{array}\right)\left(\begin{array}{ll}
0 & 0 \\
1 & 0
\end{array}\right)\left(\begin{array}{ll}
1 & 0 \\
0 & 0
\end{array}\right)\right]=-K_{0},
$$

where we have normalized the ghost correlator as in (6.21)

$$
\left\langle c\left(z_{1}\right) c\left(z_{2}\right) c\left(z_{3}\right)\right\rangle=K_{0}\left(z_{1}-z_{2}\right)\left(z_{2}-z_{3}\right)\left(z_{1}-z_{3}\right) .
$$

Eq. (6.44) corresponds to the presence of a term:

$$
-K_{0} \xi \xi^{*} \psi^{2}
$$

in the action. This in turn implies that under the gauge transformation generated by the parameter $\theta, \delta \xi=-K_{0} \theta \xi$. On the other hand, under a rigid $\mathrm{U}(1)$ gauge transformation by an infinitesimal parameter $\widetilde{\theta}$, we expect $\delta \xi=i \widetilde{\theta} \xi$. Therefore, to this order $\theta$ and $\widetilde{\theta}$ are related by simple multiplicative constant: $\theta=-i \tilde{\theta} /\left(K_{0}\right)$. In this analysis we have not been very careful in keeping track of the overall signs, which depend on the precise arrangement of the different fields in the action and the order in which we take the derivatives, since some of the fields are grassmann odd. However such sign errors will be eventually cancelled since the important quantity is the ratio of the coupling described in (6.46) and the coupling in the presence of closed string tachyon, as given in (6.47) below.

When we take into account other terms in the action containing more fields, the rigid $\mathrm{U}(1)$ gauge transformation law of $\xi$ remains unchanged, but the string field theory gauge transformation law given by the second derivative of the action with respect to $\psi^{2}$ and $\xi^{*}$, gets modified. This causes $\theta$ and $\widetilde{\theta}$ to be related by a field dependent transformation. We shall now study a particular example of this. Let us denote by $A(\omega)$ the disk amplitude (with contribution from massless internal open string states removed) with one closed string tachyon of energy $\omega$ and three open string states described by the vertex operators $I, c \partial c \partial^{2} c / 2$ and $I$, carrying the same Chan-Paton factors as in (6.44). This translates to a term in the effective action of the form:

$$
\int d \omega d P A(\omega) \Phi_{C}(\omega, P) \xi \xi^{*} \psi^{2},
$$

where $\Phi_{C}(\omega, P)$ is the closed string tachyon field of energy $\omega$ and Liouville momentum $P$. As in (6.34), the integrand in (6.47) can contain an extra factor that reduces to 1 when the closed string field is on-shell, but we shall not include this since we shall use this formula for on-shell closed string states. Adding this to (6.46) we get the following contribution to the effective action:

$$
-K_{0} \xi \xi^{*} \psi^{2}\left[1-K_{0}^{-1} \int d \omega d P A(\omega) \Phi_{C}(\omega, P)\right] .
$$


Taking derivative with respect to $\psi^{2}$ and $\xi^{*}$, we get the modified gauge transformation law of $\xi$ under the gauge transformation parameter $\theta$ :

$$
\delta \xi=-K_{0} \theta \xi\left[1-K_{0}^{-1} \int d \omega d P A(\omega) \Phi_{C}(\omega, P)\right] .
$$

Comparing this with $\delta \xi=i \widetilde{\theta} \xi$ we see that $\theta$ and $\widetilde{\theta}$ are related by:

$$
\theta=-i K_{0}^{-1} \tilde{\theta}\left[1+K_{0}^{-1} \int d \omega d P A(\omega) \Phi_{C}(\omega, P)+\cdots\right]
$$

where $\cdots$ denote higher order corrections that we shall ignore. Therefore we have

$$
\int d \theta=\text { constant } \times\left[1+K_{0}^{-1} \int d \omega d P A(\omega) \Phi_{C}(\omega, P)\right] \int d \widetilde{\theta} .
$$

Hence dividing the path integral over the open string fields by $\int d \theta$ can be regarded as a division by $\int d \widetilde{\theta}$ and a multiplication by $\left[1-K_{0}^{-1} \int d \omega d P A(\omega) \Phi_{C}(\omega, P)\right]=$ $\exp \left[-K_{0}^{-1} \int d \omega d P A(\omega) \Phi_{C}(\omega, P)\right]$ up to higher order terms. The effect of division by $\int d \tilde{\theta}$ generates a constant factor since $\int d \widetilde{\theta}$ gives the volume of the rigid $\mathrm{U}(1)$ gauge group. This can be absorbed into a redefinition of the overall normalization constant $\mathcal{N}$ that accompanies all amplitudes. Therefore we can ignore this term. This leaves us with a correction to the action of the form:

$$
-K_{0}^{-1} \int d \omega d P A(\omega) \Phi_{C}(\omega, P) .
$$

$A(\omega)$ has been evaluated in appendix $\mathrm{D}$, leading to the result:

$$
-K_{0}^{-1} A=-\frac{2}{\pi} g_{s} \sinh (\pi|\omega|)\left[\frac{\pi}{2}-\frac{\tilde{\lambda}}{2}\right] .
$$

This leads to an additional contribution to the closed string tachyon one point function due to the extra term (6.52) in the effective action. Comparing this with (6.1) we get the contribution to $g(\omega)$ from the ghost jacobian:

$$
g_{\text {ghost }}(\omega)=-\left[\frac{1}{2}-\frac{\tilde{\lambda}}{2 \pi}\right] .
$$

We note in passing that $\xi$ is the ghost field associated with the gauge transformation parameter $I \otimes\left(\begin{array}{ll}0 & 1 \\ 0 & 0\end{array}\right)$. Therefore, our calculation can also be interpreted as computing the algebra of the gauge transformations generated by $\theta$ and $\xi$, and finding appropriate redefinition of parameters that maps the algebra to the standard $\mathrm{SU}(2)$ algebra expected on a pair of D-instantons.

Before concluding this section, let us discuss one subtlety that could have affected our analysis. Usually when one tries to relate the standard gauge transformation laws of the form $\delta \xi=i \widetilde{\theta} \xi$ to the string field theory gauge transformation laws as given in (6.49), we could add to (6.49) trivial gauge transformation proportional to the equations of motion [42]. Therefore one could wonder if it is possible to change the coefficient of the second 
term inside the square bracket in (6.49) by adding such trivial gauge transformation, and modify (6.50). For this to happen, there must be a field $\chi$ whose equation of motion is proportional to $\xi \Phi_{C}(\omega, P)$. Using various conservation laws one can see that the only candidate for $\chi$ is the coefficient of the open string state $c_{1} c_{-1}|0\rangle \otimes\left(\begin{array}{ll}0 & 0 \\ 1 & 0\end{array}\right)$. However explicit computation along the line of appendix B shows that the $\chi-\xi-\Phi_{C}$ coupling on the disk vanishes, and therefore we cannot modify (6.49) by adding to it trivial gauge transformation. The vanishing of the $\chi-\xi-\Phi_{C}$ coupling can in turn be traced to the fact that $\xi$ and on-shell $\Phi_{C}$ represent BRST invariant states, whereas $\chi$ represents a BRST trivial state.

\subsection{Total contribution}

We now collect the various contributions to $g_{\text {div }}(\omega)$ as given in (6.16), (6.27), (6.43) and (6.54):

$$
\begin{aligned}
g_{\text {world }} & =\left[-\frac{2}{\pi} \widetilde{\lambda}^{2} \int_{(2 \widetilde{\lambda})^{-1}}^{1} d \beta\left(1+\beta^{2}\right)^{-1} f(\beta)^{2}+\frac{\tilde{\lambda}}{4 \pi}+\frac{1}{2} \omega^{2} \ln \frac{\alpha^{2} \widetilde{\lambda}^{2}}{4}\right], \\
g_{\psi^{1}}(\omega) & =\left[\frac{2}{\pi} \widetilde{\lambda}^{2} \int_{(2 \widetilde{\lambda})^{-1}}^{1} d \beta\left(1+\beta^{2}\right)^{-1} f(\beta)^{2}+\frac{\widetilde{\lambda}}{4 \pi}\right] \\
g_{\text {jac }}(\omega) & =-\left[\frac{1}{\pi} \widetilde{\lambda}+\omega^{2} \ln \frac{\widetilde{\lambda}^{2}}{\lambda^{2}}\right], \\
g_{\text {ghost }}(\omega) & =-\left[\frac{1}{2}-\frac{\widetilde{\lambda}}{2 \pi}\right] .
\end{aligned}
$$

Adding these, we get the following total contribution to $g_{\operatorname{div}}(\omega)$ :

$$
g_{\operatorname{div}}(\omega)=-\frac{1}{2}+\frac{1}{2} \omega^{2} \ln \frac{\lambda^{2}}{4} .
$$

Note that $g_{\text {div }}$ stands for any contribution to the annulus one point function that is not already included in $g_{\text {finite }}$ defined in (1.10). Comparing this with (1.12) we get

$$
A_{g}=-\frac{1}{2}, \quad B_{g}=\frac{1}{2} \ln \frac{\lambda^{2}}{4} .
$$

\section{Strategy for computing the correction to the D-instanton action}

In this section we shall outline the strategy for computing the remaining building block that contributes to the one instanton correction to closed string tachyon scattering to the first subleading order. This involves a zero point function on a surface of Euler number -1 . There are two different surfaces of the latter kind - a disk with two holes and a torus with a hole. If we denote the total contribution to this building block by $C g_{s}$, then the net contribution to the $(n+1)$-point tachyon amplitude from the product of $(n+1)$ disk one point functions and a zero point function on surfaces of Euler number -1 will be given by:

$$
\mathcal{N} e^{-1 / g_{s}} C g_{s} \prod_{i=1}^{n+1}\left\{2 \sinh \left(\pi\left|\omega_{i}\right|\right)\right\}
$$


Adding (7.1) to the leading order contribution $\mathcal{N} e^{-1 / g_{s}} \prod_{i}\left\{2 \sinh \left(\pi\left|\omega_{i}\right|\right)\right\}$, we see that the effect of (7.1) is to make the replacement,

$$
e^{-1 / g_{s}} \rightarrow e^{-1 / g_{s}}\left(1+C g_{s}\right)=\exp \left[-\frac{1}{g_{s}}\left\{1-C g_{s}^{2}+\mathcal{O}\left(g_{s}^{3}\right)\right\}\right] .
$$

Therefore the instanton action is modified to

$$
-\frac{1}{g_{s}}\left\{1-C g_{s}^{2}+\mathcal{O}\left(g_{s}^{3}\right)\right\} .
$$

Furthermore, this modification is independent of which amplitude we consider - this is the overall factor that multiplies the one instanton contribution to any amplitude.

Now (7.3) shows that the value of $C$ can be changed by a redefinition of $g_{s}$ of the form $g_{s} \rightarrow g_{s}\left(1+a g_{s}^{2}+\mathcal{O}\left(g_{s}^{4}\right)\right)$ for some constant $a$. In a critical string theory, $g_{s}$ is the expectation value of the dilaton field. Since different versions of open-closed string field theory are related by field redefinition, the string coupling $g_{s}$ used in different versions of string field theory are also related by redefinition of the kind described above [43, 44]. If the same story holds in two dimensional string theory, then it would seem that $C$ takes different values in different versions of string field theory. On the other hand, the matrix model yields a definite result for the tachyon scattering amplitudes, and determines $C$ via (1.13) without any ambiguity. To resolve this apparent contradiction, let us recall that while comparing the matrix model results with the string theory results, [4] made a specific choice of the relation between the matrix model parameter $\mu$ and the string coupling $g_{s}$ :

$$
2 \pi \mu=\frac{1}{g_{s}} .
$$

Let us take (7.4) as the definition of $g_{s}$, and denote by $\tilde{g}_{s}$ the string coupling in some version of string field theory. Now in the matrix model one can compute perturbative contribution to the closed string tachyon scattering amplitude with three or more tachyons in the external state. This will have an expansion in powers of $g_{s}^{2}$. In the dual string theory the same amplitude will have an expansion in powers of $\tilde{g}_{s}^{2}$. These expansion coefficients may or may not depend on the version of string field theory we use, depending on whether or not $\tilde{g}_{s}$ in different versions of string field theory are different. In either case, in any given version of string field theory, we can determine the relation between $\tilde{g}_{s}$ and $g_{s}$ by comparing any particular perturbative amplitude in the matrix model and string theory. On general ground we expect this relation to take the form ${ }^{8}$

$$
\tilde{g}_{s}=g_{s}\left(1+\mathcal{O}\left(g_{s}^{2}\right)\right)
$$

Once this is done, we can use the same string field theory to compute the zero point function on surfaces of Euler number -1, use this to determine the quantum corrected instanton

\footnotetext{
${ }^{8}$ In principle there may also be corrections of order $e^{-1 / g_{s}}$ on the right hand side of (7.5). Such a term, if present, would have given rise to an additional contribution to the three point function of order $e^{-1 / g_{s}}$ multiplying the tree level 3-point function, and spoil the agreement between the matrix model and world-sheet results at order $g_{s} e^{-1 / g_{s}}$. Therefore they do not seem to be present.
} 
action as a function of $\tilde{g}_{s}$ and then express this in terms of $g_{s}$ using the relation between $g_{s}$ and $\tilde{g}_{s}$ determined from perturbative amplitudes. This will generate an unambiguous expansion of the quantum corrected D-instanton action in powers of $g_{s}$, and determine $C$ unambiguously. Note also that the difference between $g_{s}$ and $\tilde{g}_{s}$ will not affect the functions $f$ and $g$ appearing in (1.13) since they are corrections of order $g_{s}$ to the leading order instanton induced amplitudes.

We have not computed $C$ using this procedure. However, we shall now argue that if we assume the equality of the quantum corrected D-instanton action in two dimensional string theory and the quantum corrected instanton action in the matrix model, then $C$ must vanish. In the matrix model, the dynamics is that of free fermions moving in an inverted harmonic oscillator potential. Therefore the fluctuations around the instanton solution have purely quadratic action and the instanton action does not get renormalized (except for the one loop determinant that is related to the overall normalization constant $\mathcal{N})$. Therefore the overall $\mu$ dependent multiplicative factor associated with the instanton will be given by the leading order result:

$$
e^{-2 \pi \mu}
$$

Comparing this with the right hand side of (7.2), we get

$$
2 \pi \mu=\frac{1}{g_{s}}\left\{1-C g_{s}^{2}+\mathcal{O}\left(g_{s}^{4}\right)\right\} .
$$

On the other hand, $g_{s}$ is defined by (7.4) without any correction. By comparing this with (7.7) we get

$$
C=0 \text {. }
$$

It should be noted however that the quantum corrected instanton action, by itself, is not an observable, and therefore this argument is not watertight. Nevertheless, agreement of (7.8) with the numerical results based on the comparison of the S-matrix elements in the matrix model and the world-sheet theory [16] provides a verification of the argument given above.

\section{Some open problems}

Clearly, the most urgent issue is to understand the origin of the discrepancy between the value of $A_{g}$ obtained from the string field theory computation and the result obtained by comparison with the matrix model. We discuss various possible sources of this discrepancy, in order of their likelihood.

1. The most likely explanation is that there is an error in the string field theory computation of $A_{g}$. However we have not been able to identify the origin of this error. Furthermore the string field theory results pass various internal consistency checks - the final result is independent of the string field theory parameters $\widetilde{\lambda}, \alpha$ and the function $f(\beta)$. Some further consistency checks have been described in appendix E. Although not reported here, we have also performed the computation with a different 
choice of local coordinates for the $\mathrm{O}-\mathrm{O}-\mathrm{O}$ vertex, where we take the local coordinates at the punctures at 0,1 and $\infty$ to be $\alpha z, \alpha(z-1)$ and $-\alpha / z$ respectively and then average over cyclic permutations generated by $z \rightarrow 1 /(1-z)$. Consequently the C-O$\mathrm{O}$ vertex will also have to be defined by averaging over permutations. However, the final result for $A_{g}$ does not change. We have also used the procedure of section 2.4 to reproduce our results. In fact this method can also be used to get the result without assuming $\alpha$ and / or $\lambda$ to be large, and the final result remains unchanged.

2. Another possibility is that for the D-instanton, the world-sheet theory has an additional contribution that is not related to divergences from the boundary of the moduli space. This could give an additive constant of $1 / 2$ in the computation of $g_{\text {finite }}$ and cancel the value $-1 / 2$ of $A_{g}$ that we find, restoring the equality between the string field theory results and the matrix model results. If this is the case, then it will be important to understand what the missing contribution is, since it is likely to affect the D-instanton contribution to other string theories as well.

3. A third possibility is that the matrix model result for the instanton induced scattering amplitude may have an additional term that makes the result agree with the choice $A_{g}=-1 / 2$ in (1.14). This would restore the agreement between the results of the world-sheet theory and the matrix model.

4. The final (unlikely) possibility is that the duality between the bosonic string theory and the matrix model does not hold in the form suggested in [4].

We end this paper by listing some other open problems.

1. The divergences we discussed here also exist for D-instantons in superstring theories [15] and the method described here can be used to get finite, unambiguous results. Therefore we could use this technique to find systematic $g_{s}$ expansion of D-instanton induced amplitudes. On the other hand, using non-perturbative duality symmetries, many such corrections have already been predicted [2, 45-62]. It will be interesting to verify these predictions using direct string theory computation.

2. Matrix model dual to two dimensional string theory is described by free fermions in inverted harmonic oscillator potential. The analysis of $[4,10]$ identified the two dimensional bosonic string theory with the particular vacuum where the exact energy eigenstates representing the scattering states incident from the left are filled up to the fermi level, but the scattering states incident from the right are empty. Refs. [63, 64] proposed a different correspondence where they identified two dimensional type 0B theory to the system where both sides are filled up to the Fermi level. It will be interesting to compute D-instanton induced amplitudes in type 0B theory to verify this. This would also establish that the bosonic string theory and type 0B string theory are different vacua of the same underlying theory, at least in two dimensions.

3. In our analysis we have not fixed the overall normalization constant $\mathcal{N}$ that appears in (1.13). This measures the relative normalization between the path integral measure 
in the D-instanton background and the path integral measure around the vacuum. One way to fix this will be to begin with a configuration of unstable D-branes so that both the vacuum and the D-instanton are solutions in this theory. In this case the path integral over the fields in the original theory with unstable D-branes will in principle fix the relative normalization in the path integral measure in the instanton sector and the vacuum sector. For two dimensional string theory we can in fact start with the D-instanton and regard the perturbative vacuum as the minimum of the open string tachyon potential. Therefore the integration measure of the open-closed string field theory on the D-instanton should in principle be able to determine the integration measure for closed string field theory around the perturbative vacuum. This in turn would determine the normalization constant $\mathcal{N}$.

4. Perturbative string theory is expected to be non-perturbatively incomplete. This is clearly demonstrated by the example of two dimensional bosonic and type 0B string theories, which should have the same perturbative amplitudes but different non-perturbative amplitudes. Since we now also have a fully systematic perturbation expansion for D-instanton induced amplitudes, one might ask: does this help make string theory have unique non-perturbative extension, possibly with the help of resurgence [65-67]? If not, what else is needed?

\section{Acknowledgments}

I wish to thank Bruno Balthazar, Nathan Berkovits, Carlo Maccaferri, Victor Rodriguez, Xi Yin and Barton Zwiebach for useful discussions. I specially thank Bruno Balthazar, Victor Rodriguez and Xi Yin for sending me the unpublished numerical results for $A_{f}, A_{g}$ and $C$. Part of these results was reported at the online 'Workshop on Fundamental Aspects of String Theory' organized by ICTP-SAIFR and 'Strings 2020' organized by the University of Cape Town. I thank the organizers of these conferences for giving me an opportunity to present the results there. This work was supported in part by the J. C. Bose fellowship of the Department of Science and Technology, India and the Infosys chair professorship.

\section{A Disk two point function in general coordinate system}

In this appendix we shall repeat the analysis of section 5 using a more general local coordinate system at the open string puncture of the C-O interaction vertex than what was given in (4.3).

We insert the closed string puncture at $i$ and the open string puncture at 0 on the upper half plane as usual, but choose the local coordinate at the open string puncture to be:

$$
w=\frac{\lambda z}{1+\gamma z},
$$

for some constant $\gamma$. The sewing relation $w w^{\prime}=-q$ now gives

$$
\lambda^{2} \frac{z}{1+\gamma z} \frac{z^{\prime}}{1+\gamma z^{\prime}}=-q .
$$


In the $z$ plane the closed string punctures at $z=i$ and $z^{\prime}=i$ are located at, respectively,

$$
z_{1}=i, \quad z_{2}=i u \frac{1+i \gamma}{1-i u \gamma+u \gamma^{2}}, \quad u=\frac{q}{\lambda^{2}}, \quad 0 \leq u \leq \lambda^{-2} .
$$

We now introduce a new coordinate $\hat{z}=(z-a) /(1+a z)$ for some real constant $a$. This maps the closed string punctures to

$$
\hat{z}_{1}=i, \quad \hat{z}_{2}=\frac{z_{2}-a}{1+a z_{2}}
$$

Our goal is to adjust $a$ so that $\hat{z}_{2}$ is purely imaginary and then identify $\hat{z}_{2}$ as $i y$. Since it follows from the analysis of section 3 that we need the relation between $u$ and $y$ to the first subleading order in the expansion in powers of $u$, we can work only to this order. We get

$$
a=-\gamma u-\gamma u^{2}+\gamma^{3} u^{2}+\mathcal{O}\left(u^{3}\right), \quad y=u-2 \gamma^{2} u^{2}+\mathcal{O}\left(u^{3}\right) .
$$

Therefore the range $0 \leq u \leq \lambda^{-2}$ translates to $0 \leq y \leq \lambda^{-2}-2 \gamma^{2} \lambda^{-4}$. In this range we change variable from $y$ to $q=u \lambda^{2}$ in (5.3) and apply the replacement rule (2.11), and in the rest of the range of integration we carry out the $y$ integration explicitly. This gives the following result for (5.3):

$$
\begin{aligned}
\frac{1}{2} \int_{0}^{1} d y y^{-2}\left(1+2 \omega_{1} \omega_{2} y\right) & =\frac{1}{2} \int_{0}^{1} d q\left\{\lambda^{2} q^{-2}+2 \omega_{1} \omega_{2} q^{-1}\right\}+\frac{1}{2} \int_{\lambda^{-2}-2 \gamma^{2} \lambda^{-4}}^{1} d y y^{-2}\left(1+2 \omega_{1} \omega_{2} y\right) \\
& \rightarrow-\frac{1}{2} \lambda^{2}+\frac{1}{2} \frac{\lambda^{2}}{1-2 \gamma^{2} \lambda^{-2}}-\frac{1}{2}+\omega_{1} \omega_{2} \ln \lambda^{2} \\
& =\gamma^{2}-\frac{1}{2}\left\{1-2 \omega_{1} \omega_{2} \ln \lambda^{2}\right\}
\end{aligned}
$$

where in all the steps we have ignored terms containing negative powers of $\lambda$. This differs from (5.5) by the additive term $\gamma^{2}$. We shall now show that this is cancelled by the $\psi^{1}$ exchange contribution to figure 3(a).

As usual, in order to avoid normalization issues, it will be simplest to compare the $\psi^{1}$ exchange contribution with the tachyon exchange contribution, given by the first term in the second line of (A.6). Since it follows from (6.23) that the $\psi^{1}$ propagator is $1 / 2$ times the tachyon propagator, and we see from the first term in the second line of (A.6) that the tachyon exchange contribution is $-\lambda^{2} / 2$, we can express the $\psi^{1}$ exchange contribution as:

$$
-\frac{1}{4} \lambda^{2}\left(\frac{C_{\Phi \psi^{1}}}{C_{\Phi \psi^{0}}}\right)^{2},
$$

where $C_{\Phi \psi^{0}}$ and $C_{\Phi \psi^{1}}$ are respectively the disk two point function of a closed string tachyon and an open string tachyon associated with the vertex operator $c$, and the disk two point function of a closed string tachyon and the open string $\psi^{1}$ field associated with the vertex operator $\partial c$. To compute these, let us express (A.1) as:

$$
z=F(w), \quad F(w)=\frac{w}{\lambda-\gamma w} .
$$


This gives the conformal transforms of the open string tachyon and $\psi^{1}$ vertex operators:

$$
F \circ c(0)=\left(F^{\prime}(0)\right)^{-1} c(F(0))=\lambda c(0), \quad F \circ \partial c(0)=\partial c(F(0))-\frac{F^{\prime \prime}(0)}{F^{\prime}(0)^{2}} c(F(0))=\partial c(0)-2 \gamma c(0) \text {. }
$$

Now as shown in (5.6), the correlation function of a closed string tachyon vertex operator at $i$ and $\partial c(0)$ vanishes. Therefore we see from (A.9) that $C_{\Phi \psi^{0}} / C_{\Phi \psi^{1}}=-2 \gamma \lambda^{-1}$. Substituting this into (A.7) we arrive at the $\psi^{1}$ exchange contribution:

$$
-\frac{1}{4} \lambda^{2}\left(\frac{2 \gamma}{\lambda}\right)^{2}=-\gamma^{2}
$$

This exactly cancels the first term on the right hand side of (A.6), giving back the original result (5.7).

\section{B $\psi^{1}$ exchange contribution to the annulus one point function}

In this appendix we shall compute the $\psi^{1}$ exchange contribution to the annulus one point function discussed in section 6.2. The relevant diagrams are the ones shown in figure 9 .

Let us begin with figure $9(\mathrm{~b})$. This involves a C-O-O interaction vertex with both O's representing the field $\psi^{1}$. Let $z$ be the coordinate on the upper half plane in which the closed string puncture is situated at $i$ and the open string punctures are situated at $z_{1}=-\beta$ and $z_{2}=\beta$. Let $w_{1}$ and $w_{2}$ be the local coordinates at the open string punctures, related to $z$ via the relations

$$
z=F_{a}\left(w_{a}, \beta\right) .
$$

According to (6.26), the amplitude is given by:

$$
-K_{1} \int d \beta \sum_{a=1}^{2} \oint_{a} \frac{\partial F_{a}}{\partial \beta} d z\left\langle b(z) F_{1} \circ \partial c(0) F_{2} \circ \partial c(0) c \bar{c} V_{T}(i)\right\rangle
$$

where $c \bar{c} V_{T}$ denotes the vertex operator of the closed string tachyon of energy $\omega, \oint_{a}$ is the anti-clockwise contour around $z_{a}=F_{a}(0 ; \beta)$ and $K_{1}$ is a normalization constant. We shall assume that $\oint$ includes a $1 /(2 \pi i)$ factor. $F_{a} \circ \partial c(0)$ denotes the conformal transform of $\partial c(0)$ by $F_{a}$.

Let $F_{a}\left(w_{a}, \beta\right)$ have an expansion of the form:

$$
F_{a}\left(w_{a}, \beta\right)=f_{a}(\beta)+g_{a}(\beta) w_{a}+\frac{1}{2} h_{a}(\beta) w_{a}^{2}+\mathcal{O}\left(w_{a}^{3}\right) .
$$

Then the locations of the punctures are given by,

$$
z_{a}=F_{a}(0, \beta)=f_{a}(\beta) .
$$

Furthermore, $F_{a} \circ c(0)$ and $F_{a} \circ \partial c(0)$ can be found from (A.9) and (B.3):

$$
F_{a} \circ c(0)=g_{a}(\beta)^{-1} c\left(z_{a}\right), \quad F_{a} \circ \partial c(0)=\partial c\left(z_{a}\right)-\frac{h_{a}(\beta)}{g_{a}(\beta)^{2}} c\left(z_{a}\right) .
$$


Let us introduce another constant $K_{2}$ via the relation:

$$
\left\langle c(z) c \bar{c} V_{T}(i)\right\rangle=K_{2}\left(z^{2}+1\right), \quad\left\langle\partial c(z) c \bar{c} V_{T}(i)\right\rangle=2 K_{2} z
$$

Then (B.2) takes the form,

$$
\begin{aligned}
-K_{1} \int d \beta \sum_{a=1}^{2} \oint_{a}\langle & {\left[\frac{\partial f_{a}}{\partial \beta}+\frac{1}{g_{a}} \frac{\partial g_{a}}{\partial \beta}\left(z-z_{a}\right)+\mathcal{O}\left(\left(z-z_{a}\right)^{2}\right)\right] b(z) d z\left(\partial c\left(z_{1}\right)-g_{1}^{-2} h_{1} c\left(z_{1}\right)\right) } \\
& \left.\left(\partial c\left(z_{2}\right)-g_{2}^{-2} h_{2} c\left(z_{2}\right)\right) c \bar{c} V_{T}(i)\right\rangle \\
=-K_{1} K_{2} \int d \beta[ & \left\{\frac{1}{g_{1}} \frac{\partial g_{1}}{\partial \beta}-\frac{h_{1}}{g_{1}^{2}} \frac{\partial f_{1}}{\partial \beta}\right\}\left\{2 f_{2}-g_{2}^{-2} h_{2}\left(f_{2}^{2}+1\right)\right\} \\
& \left.-\left\{\frac{1}{g_{2}} \frac{\partial g_{2}}{\partial \beta}-\frac{h_{2}}{g_{2}^{2}} \frac{\partial f_{2}}{\partial \beta}\right\}\left\{2 f_{1}-g_{1}^{-2} h_{1}\left(f_{1}^{2}+1\right)\right\}\right] .
\end{aligned}
$$

In arriving at the right hand side of (B.7) we have used the operator product expansion $b(z) c\left(z_{a}\right) \sim\left(z-z_{a}\right)^{-1}, b(z) \partial c\left(z_{a}\right) \sim\left(z-z_{a}\right)^{-2}$, where $\sim$ denotes equality up to non-singular terms.

Now we have, from (4.12),

$$
\begin{aligned}
& w_{1}=\alpha \widetilde{\lambda} \frac{4 \widetilde{\lambda}^{2}+1}{4 \widetilde{\lambda}^{2}} \frac{z+\beta}{(1-\beta z)-\widetilde{\lambda} f(\beta)(z+\beta)}, \\
& w_{2}=\alpha \widetilde{\lambda} \frac{4 \widetilde{\lambda}^{2}+1}{4 \widetilde{\lambda}^{2}} \frac{z-\beta}{(1+\beta z)+\widetilde{\lambda} f(\beta)(z-\beta)} .
\end{aligned}
$$

Inverting these relations and expanding them in powers of $w_{a}$, we get

$$
\begin{aligned}
& z \equiv F_{1}\left(w_{1}, \beta\right)=-\beta+\frac{4 \widetilde{\lambda}^{2}}{4 \widetilde{\lambda}^{2}+1} \frac{1+\beta^{2}}{\alpha \widetilde{\lambda}} w_{1}-\left(\frac{4 \widetilde{\lambda}^{2}}{4 \widetilde{\lambda}^{2}+1}\right)^{2} \frac{\beta+\widetilde{\lambda} f}{(\alpha \widetilde{\lambda})^{2}}\left(1+\beta^{2}\right) w_{1}^{2}+\mathcal{O}\left(w_{1}^{3}\right), \\
& z \equiv F_{2}\left(w_{2}, \beta\right)=\beta+\frac{4 \widetilde{\lambda}^{2}}{4 \widetilde{\lambda}^{2}+1} \frac{1+\beta^{2}}{\alpha \widetilde{\lambda}} w_{2}+\left(\frac{4 \widetilde{\lambda}^{2}}{4 \widetilde{\lambda}^{2}+1}\right)^{2} \frac{\beta+\widetilde{\lambda} f}{(\alpha \widetilde{\lambda})^{2}}\left(1+\beta^{2}\right) w_{2}^{2}+\mathcal{O}\left(w_{2}^{3}\right) .
\end{aligned}
$$

Comparing these with (B.3) we get,

$$
\begin{array}{ll}
z_{1}=f_{1}(\beta)=-\beta, & g_{1}(\beta)=\frac{4 \widetilde{\lambda}^{2}}{4 \widetilde{\lambda}^{2}+1} \frac{1+\beta^{2}}{\alpha \widetilde{\lambda}}, \quad h_{1}(\beta)=-2\left(\frac{4 \widetilde{\lambda}^{2}}{4 \widetilde{\lambda}^{2}+1}\right)^{2} \frac{\beta+\widetilde{\lambda} f}{(\alpha \widetilde{\lambda})^{2}}\left(1+\beta^{2}\right), \\
z_{2}=f_{2}(\beta)=\beta, \quad g_{2}(\beta)=\frac{4 \widetilde{\lambda}^{2}}{4 \widetilde{\lambda}^{2}+1} \frac{1+\beta^{2}}{\alpha \widetilde{\lambda}}, \quad h_{2}(\beta)=2\left(\frac{4 \widetilde{\lambda}^{2}}{4 \widetilde{\lambda}^{2}+1}\right)^{2} \frac{\beta+\widetilde{\lambda} f}{(\alpha \widetilde{\lambda})^{2}}\left(1+\beta^{2}\right) .
\end{array}
$$

Substituting these into (B.7) and multiplying the result by the $\psi^{1}$ propagator $K_{0}^{-1} / 2$ obtained from (6.23), we get the contribution to figure 9(b):

$$
-4 K_{1} K_{2} K_{0}^{-1} \int_{1 /(2 \widetilde{\lambda})}^{1} \frac{d \beta}{1+\beta^{2}} \widetilde{\lambda}^{2} f(\beta)^{2} .
$$


We shall now determine the constant $K_{1} K_{2} K_{0}^{-1}$. For this let us consider the effect of replacing $\psi^{1}$ by the open string tachyon in figure $9(\mathrm{~b})$. The analysis proceeds as above, except that instead of the vertex operator of $\psi^{1}$, we insert the tachyon vertex operators as given in the first equation of (B.5). The analog of (B.7) now takes the form:

$$
\begin{aligned}
& -K_{1} \int d \beta \sum_{a=1}^{2} \oint\left\langle\left[\frac{\partial f_{a}}{\partial \beta}+\frac{1}{g_{a}} \frac{\partial g_{a}}{\partial \beta}\left(z-z_{a}\right)+\mathcal{O}\left(z-z_{a}\right)^{2}\right] b(z) d z g_{1}^{-1} c\left(z_{1}\right) g_{2}^{-1} c\left(z_{2}\right) c \bar{c} V_{T}(i)\right\rangle \\
& =-K_{1} K_{2} \int d \beta\left[\frac{\partial f_{1}}{\partial \beta}\left(1+z_{2}^{2}\right)-\frac{\partial f_{2}}{\partial \beta}\left(1+z_{1}^{2}\right)\right] g_{1}^{-1} g_{2}^{-1} .
\end{aligned}
$$

Multiplying this by the tachyon propagator $K_{0}^{-1}$ and using (B.10), we get the contribution to figure 9 (b) with $\psi^{1}$ replaced by the open string tachyon:

$$
2 K_{1} K_{2} K_{0}^{-1} \alpha^{2} \widetilde{\lambda}^{2}\left(\frac{4 \widetilde{\lambda}^{2}+1}{4 \widetilde{\lambda}^{2}}\right)^{2} \int_{1 /(2 \widetilde{\lambda})}^{1} \frac{d \beta}{1+\beta^{2}} .
$$

On the other hand, the tachyon contribution, computed from the first term in the last line of (6.9), is given by:

$$
-\frac{2}{\pi} g_{s} \sinh (\pi|\omega|) \alpha^{2} \widetilde{\lambda}^{2}\left(1+\frac{1}{4 \widetilde{\lambda}^{2}}\right)^{2} \int_{1 /(2 \widetilde{\lambda})}^{1} \frac{d \beta}{1+\beta^{2}} .
$$

Note that we have put back a factor of $2 g_{s} \sinh (\pi|\omega|)$ that was factored out from (6.1) in defining $g(\omega)$. Comparison of (B.13) and (B.14) now gives

$$
K_{1} K_{2} K_{0}^{-1}=-\frac{g_{s}}{\pi} \sinh (\pi|\omega|) .
$$

Substituting this into (B.11) we get the $\psi^{1}$ exchange contribution to figure 9 (b):

$$
\frac{4}{\pi} g_{s} \sinh (\pi|\omega|) \int_{(2 \widetilde{\lambda})^{-1}}^{1} d \beta\left(1+\beta^{2}\right)^{-1} \widetilde{\lambda}^{2} f(\beta)^{2} .
$$

The contribution from figure 9 (a) may be analyzed similarly. The main difference is that the local coordinates $w_{a}$ now have a different relation to the coordinate $z$ in the upper half plane. We have, from (4.9), (4.10),

$$
w_{1}=-2 \alpha \frac{z+\beta}{z-3 \beta}, \quad w_{2}=2 \alpha \frac{z-\beta}{z+3 \beta}, \quad \beta \equiv \frac{q}{2 \widetilde{\lambda}} .
$$

Inverting these relations we can identify the functions $F_{1}$ and $F_{2}$ :

$$
\begin{aligned}
& z \equiv F_{1}\left(w_{1}, \beta\right)=-\beta+\frac{2 \beta}{\alpha} w_{1}-\frac{\beta}{\alpha^{2}} w_{1}^{2}+\mathcal{O}\left(w_{1}^{3}\right), \\
& z \equiv F_{2}\left(w_{2}, \beta\right)=\beta+\frac{2 \beta}{\alpha} w_{2}+\frac{\beta}{\alpha^{2}} w_{2}^{2}+\mathcal{O}\left(w_{1}^{3}\right) .
\end{aligned}
$$

Comparison with (B.3) now gives:

$$
f_{1}=-\beta, \quad g_{1}=\frac{2 \beta}{\alpha}, \quad h_{1}=-\frac{2 \beta}{\alpha^{2}}, \quad f_{2}=\beta, \quad g_{2}=\frac{2 \beta}{\alpha}, \quad h_{2}=\frac{2 \beta}{\alpha^{2}} .
$$


Substituting this into (B.7), multiplying this by the $\psi^{1}$ propagator $K_{0}^{-1} / 2$ and integrating over $\beta$, we get the contribution to figure (9)(a):

$$
-K_{1} K_{2} K_{0}^{-1} \int_{0}^{1 /(2 \widetilde{\lambda})} d \beta\left[\frac{3}{4}-\frac{1}{4 \beta^{2}}\right]=\frac{g_{s}}{2 \pi} \sinh (\pi|\omega|) \tilde{\lambda}
$$

where in the last step we have used (B.15), replaced $\beta$ by $q /(2 \widetilde{\lambda})$ according to (4.9) and used the replacement rule $(2.11)$, throwing away terms of order $1 / \widetilde{\lambda}$.

Adding (B.16) and (B.20), and dividing the result by $2 g_{s} \sinh (\pi|\omega|)$, we get the total $\psi^{1}$ exchange contribution to $g(\omega)$ :

$$
g_{\psi^{1}}(\omega)=\left[\frac{2}{\pi} \int_{(2 \widetilde{\lambda})^{-1}}^{1} d \beta\left(1+\beta^{2}\right)^{-1} \widetilde{\lambda}^{2} f(\beta)^{2}+\frac{\widetilde{\lambda}}{4 \pi}\right]
$$

\section{Computation related to collective mode redefinition}

As discussed in section 6.3, in order to find the field redefinition that relates the open string zero mode associated with the vertex operator $c \partial X$ to the collective mode associated with time translation of the D-instanton, we need to evaluate the 'divergent' part of the contribution to the disk amplitude with a pair of closed string tachyons of energies $\omega_{1}$ and $\omega_{2}$ and an open string zero mode field described by the vertex operator $c \partial X$. According to $(6.30)$, the relevant integral is:

$$
\begin{aligned}
\int_{-\infty}^{\infty} d x \int_{0}^{1} d y \frac{1}{2 \pi} & {\left[-\frac{\omega_{1}}{x-i}+\frac{\omega_{1}}{x+i}-\frac{\omega_{2}}{x-i y}+\frac{\omega_{2}}{x+i y}\right] } \\
& \times 4 g_{s} \sinh \left(\pi\left|\omega_{1}\right|\right) \sinh \left(\pi\left|\omega_{2}\right|\right)\left[\frac{1}{2} y^{-2}\left(1+2 \omega_{1} \omega_{2} y\right)\right]
\end{aligned}
$$

and we have to evaluate the contribution to this integral from the different regions shown in figure 5, associated with the Feynman diagrams of figure 4. In this appendix we shall carry out this computation.

First we evaluate the contribution to (C.1) from region IV in figure 5. There are no singularities from this region and we need to evaluate the integral explicitly. We see from (4.55) that the $x$ integration is unrestricted and the $y$ integration range may be expressed as:

$$
1 \geq y \geq \tilde{\lambda}^{-2}-\tilde{\lambda}^{-4} \Delta(x)
$$

where

$$
\Delta(x)=F(x)^{2}+\frac{1}{4}
$$

In the above we have ignored fractional corrections of order $\widetilde{\lambda}^{-4}$. Our strategy for evaluating the integral is to divide region IV into two parts: $y \geq \widetilde{\lambda}^{-2}$, and $y$ in the range $\left(\widetilde{\lambda}^{-2}-\right.$ $\left.\tilde{\lambda}^{-4} \Delta(x), \tilde{\lambda}^{-2}\right)$. Since the latter range is small, we can evaluate the $y$ integral by replacing $y$ by $\widetilde{\lambda}^{-2}$ in the integrand and then multiplying the integrand by $\widetilde{\lambda}^{-4} \Delta(x)$. If we denote 
these two integrals by $I_{1}$ and $I_{2}$, then we have:

$$
\begin{aligned}
I_{1}= & \int_{-\infty}^{\infty} d x \int_{\widetilde{\lambda}^{-2}}^{1} d y \frac{1}{2 \pi}\left[-\frac{\omega_{1}}{x-i}+\frac{\omega_{1}}{x+i}-\frac{\omega_{2}}{x-i y}+\frac{\omega_{2}}{x+i y}\right] \\
& \times 2 g_{s} \sinh \left(\pi\left|\omega_{1}\right|\right) \sinh \left(\pi\left|\omega_{2}\right|\right) y^{-2}\left(1+2 \omega_{1} \omega_{2} y\right) \\
= & -i\left(\omega_{1}+\omega_{2}\right) 2 g_{s} \sinh \left(\pi\left|\omega_{1}\right|\right) \sinh \left(\pi\left|\omega_{2}\right|\right) \int_{\widetilde{\lambda}^{-2}}^{1} d y y^{-2}\left(1+2 \omega_{1} \omega_{2} y\right) \\
= & -2 i\left(\omega_{1}+\omega_{2}\right) g_{s} \sinh \left(\pi\left|\omega_{1}\right|\right) \sinh \left(\pi\left|\omega_{2}\right|\right)\left\{\widetilde{\lambda}^{2}-1+2 \omega_{1} \omega_{2} \ln \widetilde{\lambda}^{2}\right\} .
\end{aligned}
$$

On the other hand, we get,

$$
\begin{aligned}
I_{2}= & \int_{-\infty}^{\infty} d x \widetilde{\lambda}^{-4} \Delta(x) \frac{1}{2 \pi}\left[-\frac{\omega_{1}}{x-i}+\frac{\omega_{1}}{x+i}-\frac{\omega_{2}}{x-i \widetilde{\lambda}^{-2}}+\frac{\omega_{2}}{x+i \widetilde{\lambda}^{-2}}\right] \\
& \times 2 g_{s} \sinh \left(\pi\left|\omega_{1}\right|\right) \sinh \left(\pi\left|\omega_{2}\right|\right) \widetilde{\lambda}^{4}\left(1+2 \omega_{1} \omega_{2} \widetilde{\lambda}^{-2}\right) \\
= & 4 i g_{s} \sinh \left(\pi\left|\omega_{1}\right|\right) \sinh \left(\pi\left|\omega_{2}\right|\right)\left(1+2 \omega_{1} \omega_{2} \widetilde{\lambda}^{-2}\right) \int_{-\infty}^{\infty} d x \Delta(x) \frac{1}{2 \pi}\left[-\frac{\omega_{1}}{x^{2}+1}-\frac{\omega_{2} \widetilde{\lambda}^{-2}}{x^{2}+\widetilde{\lambda}^{-4}}\right] .
\end{aligned}
$$

Making a change of variable $x \rightarrow \widetilde{\lambda}^{-2} / x$ in the second term, and using (4.54), we can express this as

$$
I_{2}=-\frac{2 i}{\pi}\left(\omega_{1}+\omega_{2}\right) g_{s} \sinh \left(\pi\left|\omega_{1}\right|\right) \sinh \left(\pi\left|\omega_{2}\right|\right) \int_{-\infty}^{\infty} d x \Delta(x)\left[\frac{1}{x^{2}+1}\right],
$$

where we have ignored fractional errors of order $\widetilde{\lambda}^{-2}$. This can be justified as follows. $F(x)$ is given by (4.45), (4.48), (4.53). Since $f(\beta)$ in these expressions are bounded from above by $1, F(x)$ and hence $\Delta(x)$ defined in (C.3) are also bounded. This shows that $I_{2}$ is a finite integral, and justifies working to leading order in the expansion in powers of $\tilde{\lambda}^{-2}$ for this integral. Using (4.45), (4.48), (4.53), (C.3), and ignoring terms containing negative powers of $\widetilde{\lambda}$, (C.6) may be expressed as:

$$
I_{2}=-\frac{8 i}{\pi}\left(\omega_{1}+\omega_{2}\right) g_{s} \sinh \left(\pi\left|\omega_{1}\right|\right) \sinh \left(\pi\left|\omega_{2}\right|\right) \int_{\frac{1}{2 \widetilde{\lambda}}}^{1} d \beta\left(1+\beta^{2}\right)^{-1}\left\{f(\beta)^{2}+\frac{1}{4}\right\} .
$$

Next we consider regions III and III'. In these regions, we need to change variable from $(x, y)$ to $(u, \beta)$ according to (4.49), (4.50), and then integrate over the range (4.43). It will be more convenient to trade in the variable $\beta$ for $\xi$, and introduce the function $\phi(\xi)$ via the relations:

$$
\xi= \pm \frac{2 \beta}{1-\beta^{2}}, \quad \phi(\xi)=f(\beta),
$$

so that (4.49), (4.50) may be combined into the equation:

$$
y=\frac{u}{\widetilde{\lambda}}\left\{1+u^{2} \phi(\xi)^{2}\right\}^{-1}, \quad x=\xi-u^{2} \widetilde{\lambda}^{-1} \phi(\xi) .
$$

In these variables the range (4.43) translates to:

$$
0 \leq u \leq \frac{4 \widetilde{\lambda}}{4 \widetilde{\lambda}^{2}+1}, \quad \frac{4 \widetilde{\lambda}}{4 \widetilde{\lambda}^{2}-1} \leq|\xi|<\infty .
$$


We also have

$$
d x \wedge d y=\tilde{\lambda}^{-1} d \xi \wedge d u\left\{1-3 u^{2} \phi(\xi)^{2}\right\}
$$

ignoring fractional corrections of order higher than $\widetilde{\lambda}^{-2}$. This may be justified by noting that after using the replacement rule (3.4), the leading divergent term in the integral (C.1) is of order $\widetilde{\lambda}^{2}$. Therefore fractional corrections of order higher than $\widetilde{\lambda}^{-2}$ will be suppressed in the large $\tilde{\lambda}$ limit. Using (C.9)-(C.11) we may now express the contribution to the integral (C.1) from regions III and III' as:

$$
\begin{aligned}
I_{3}= & -2 i \iint_{\mathrm{III}+\mathrm{III}} d x d y \frac{1}{2 \pi}\left[\frac{\omega_{1}}{x^{2}+1}+\frac{\omega_{2} y}{x^{2}+y^{2}}\right] \\
& \times 4 g_{s}\left[\frac{1}{2} \sinh \left(\pi\left|\omega_{1}\right|\right) \sinh \left(\pi\left|\omega_{2}\right|\right) y^{-2}\left(1+2 \omega_{1} \omega_{2} y\right)\right] \\
= & -\frac{2}{\pi} i \widetilde{\lambda} g_{s} \sinh \left(\pi\left|\omega_{1}\right|\right) \sinh \left(\pi\left|\omega_{2}\right|\right) \int_{|\xi| \geq 4 \widetilde{\lambda} /\left(4 \widetilde{\lambda}^{2}-1\right)} d \xi \int_{0}^{4 \widetilde{\lambda} /\left(4 \widetilde{\lambda}^{2}+1\right)} d u \\
& \times\left\{\frac{\omega_{1}}{\left\{\xi-u^{2} \widetilde{\lambda}^{-1} \phi(\xi)\right\}^{2}+1}+\frac{\omega_{2} u \widetilde{\lambda}^{-1}\left\{1+u^{2} \phi(\xi)^{2}\right\}^{-1}}{\left\{\xi-u^{2} \widetilde{\lambda}^{-1} \phi(\xi)\right\}^{2}+u^{2} \widetilde{\lambda}^{-2}\left\{1+u^{2} \phi(\xi)^{2}\right\}^{-2}}\right\} \\
& \times u^{-2}\left\{1+u^{2} \phi(\xi)^{2}\right\}^{2}\left[1+2 \omega_{1} \omega_{2} u \widetilde{\lambda}^{-1}\left\{1+u^{2} \phi(\xi)^{2}\right\}^{-1}\right]\left\{1-3 u^{2} \phi(\xi)^{2}\right\}
\end{aligned}
$$

Note that the $\xi$ integration range includes both positive and negative $\xi$. We now expand the integrand in a Laurent series expansion in $u$ and use the replacement rules:

$$
\int_{0}^{4 \widetilde{\lambda} /\left(4 \widetilde{\lambda}^{2}+1\right)} d u u^{-2} \rightarrow-\frac{4 \widetilde{\lambda}^{2}+1}{4 \widetilde{\lambda}}, \quad \int_{0}^{4 \widetilde{\lambda} /\left(4 \widetilde{\lambda}^{2}+1\right)} d u u^{-1} \rightarrow 0 .
$$

Integrals with non-negative powers of $u$ in the integrand may be carried out explicitly. This gives

$$
I_{3}=\frac{2}{\pi} i \widetilde{\lambda} g_{s} \sinh \left(\pi\left|\omega_{1}\right|\right) \sinh \left(\pi\left|\omega_{2}\right|\right) \int_{|\xi| \geq 4 \widetilde{\lambda} /\left(4 \widetilde{\lambda}^{2}-1\right)} d \xi \frac{\omega_{1}}{\xi^{2}+1}\left[\frac{4 \widetilde{\lambda}^{2}+1}{4 \widetilde{\lambda}}+\frac{4 \widetilde{\lambda}}{4 \widetilde{\lambda}^{2}+1} \phi(\xi)^{2}\right]
$$

where we have thrown away the terms that would vanish in the large $\tilde{\lambda}$ limit. Using (C.8) this may be written as:

$$
I_{3}=\frac{8}{\pi} i \omega_{1} g_{s} \sinh \left(\pi\left|\omega_{1}\right|\right) \sinh \left(\pi\left|\omega_{2}\right|\right) \int_{\frac{1}{2 \widetilde{\lambda}}}^{1} d \beta \frac{1}{\beta^{2}+1}\left[\frac{4 \widetilde{\lambda}^{2}+1}{4}+f(\beta)^{2}\right] .
$$

The change of variables in region II can be found in (4.42) and (4.46). Let us first define

$$
\tilde{x}=-y / x
$$

and express (4.42) and (4.46) as:

$$
y=\frac{u}{\widetilde{\lambda}}\left\{1+u^{2} \phi(\xi)^{2}\right\}^{-1}, \quad \tilde{x}=\xi-u^{2} \widetilde{\lambda}^{-1} \phi(\xi),
$$


where $\xi$ and $\phi(\xi)$ have been defined in (C.8). As already discussed in section 4, under (C.16)

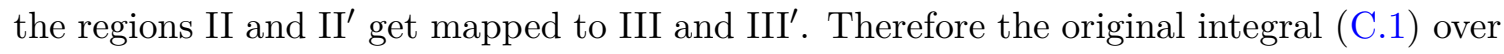
regions II and $\mathrm{II}^{\prime}$ can be rewritten as follows:

$$
\begin{aligned}
I_{4}= & -2 i \iint_{\mathrm{II}+\mathrm{II}^{\prime}} d x d y \frac{1}{2 \pi}\left[\frac{\omega_{1}}{x^{2}+1}+\frac{\omega_{2} y}{x^{2}+y^{2}}\right] \\
& \times 4 g_{s}\left[\frac{1}{2} \sinh \left(\pi\left|\omega_{1}\right|\right) \sinh \left(\pi\left|\omega_{2}\right|\right) y^{-2}\left(1+2 \omega_{1} \omega_{2} y\right)\right] \\
= & -2 i \iint_{\mathrm{III}+\mathrm{III}} d \tilde{x} d y \frac{1}{2 \pi}\left[\frac{\omega_{1} y}{\tilde{x}^{2}+y^{2}}+\frac{\omega_{2}}{\tilde{x}^{2}+1}\right] \\
& \times 2 g_{s} \sinh \left(\pi\left|\omega_{1}\right|\right) \sinh \left(\pi\left|\omega_{2}\right|\right) y^{-2}\left(1+2 \omega_{1} \omega_{2} y\right) .
\end{aligned}
$$

Eq. (C.17), (C.18) have the same form as (C.9), (C.12), with $\omega_{1} \leftrightarrow \omega_{2}$ and $x$ replaced by $\tilde{x}$. Therefore this integral can be analyzed in the same way, leading to the analog of (C.15):

$$
I_{4}=\frac{8}{\pi} i \omega_{2} g_{s} \sinh \left(\pi\left|\omega_{1}\right|\right) \sinh \left(\pi\left|\omega_{2}\right|\right) \int_{\frac{1}{2 \widetilde{\lambda}}}^{1} d \beta \frac{1}{\beta^{2}+1}\left[\frac{4 \widetilde{\lambda}^{2}+1}{4}+f(\beta)^{2}\right] .
$$

Finally let us turn to regions I and $\mathrm{I}^{\prime}$. The change of variables is given in (4.24), (4.25) for region I

$y=u_{1} u_{2}\left\{1-\frac{u_{1}^{2}}{4}-\frac{u_{2}^{2}}{4}+\mathcal{O}\left(u_{1}^{4}, u_{2}^{4}, u_{1}^{2} u_{2}^{2}\right)\right\}, \quad x=-u_{1}\left\{1+\frac{u_{1}^{2}}{4}-\frac{u_{2}^{2}}{2}+\mathcal{O}\left(u_{1}^{4}, u_{2}^{4}, u_{1}^{2} u_{2}^{2}\right)\right\}$,

and (4.31), (4.32) in region $\mathrm{I}^{\prime}$,

$$
y=u_{1} u_{2}\left\{1-\frac{u_{1}^{2}}{4}-\frac{u_{2}^{2}}{4}+\mathcal{O}\left(u_{1}^{4}, u_{2}^{4}, u_{1}^{2} u_{2}^{2}\right)\right\}, \quad x=u_{1}\left\{1+\frac{u_{1}^{2}}{4}-\frac{u_{2}^{2}}{2}+\mathcal{O}\left(u_{1}^{4}, u_{2}^{4}, u_{1}^{2} u_{2}^{2}\right)\right\} .
$$

This gives:

$$
d x d y=u_{1} d u_{1} d u_{2}\left\{1+\frac{u_{1}^{2}}{2}-\frac{u_{2}^{2}}{4}\right\} .
$$

Using this, the contribution to the integral from this region may be expressed as:

$$
\begin{aligned}
I_{5}= & -2 i \iint_{\mathrm{I}+\mathrm{I}^{\prime}} d x d y \frac{1}{2 \pi}\left[\frac{\omega_{1}}{x^{2}+1}+\frac{\omega_{2} y}{x^{2}+y^{2}}\right] \\
& \times 4 g_{s}\left[\frac{1}{2} \sinh \left(\pi\left|\omega_{1}\right|\right) \sinh \left(\pi\left|\omega_{2}\right|\right) y^{-2}\left(1+2 \omega_{1} \omega_{2} y\right)\right] \\
= & -\frac{4}{\pi} i g_{s} \sinh \left(\pi\left|\omega_{1}\right|\right) \sinh \left(\pi\left|\omega_{2}\right|\right) \int_{0}^{\widetilde{\lambda}^{-1}} d u_{1} \int_{0}^{\widetilde{\lambda}^{-1}} d u_{2} u_{1}^{-2} u_{2}^{-2} d u_{1} d u_{2} \\
& \times\left[\omega_{1} u_{1}\left\{1+\frac{u_{2}^{2}}{4}\right\}+\omega_{2} u_{2}\left\{1+\frac{u_{1}^{2}}{4}\right\}\right]\left[1+2 \omega_{1} \omega_{2} u_{1} u_{2}\left\{1-\frac{u_{1}^{2}}{4}-\frac{u_{2}^{2}}{4}\right\}\right] .
\end{aligned}
$$

We evaluate this by using the replacement rules:

$$
\int_{0}^{\widetilde{\lambda}^{-1}} d u_{i} u_{i}^{-2} \rightarrow-\tilde{\lambda}, \quad \int_{0}^{\tilde{\lambda}^{-1}} d u_{i} u_{i}^{-1} \rightarrow 0
$$


This leads to

$$
I_{5}=0
$$

Finally we note that the $\psi^{1}$ exchange contribution to any of the diagrams in figure 4(a), (b) or (c) vanishes, since each internal open string propagator is connected at least at one end to an open-closed two point interaction vertex, and for such vertices, the contribution vanishes according to (5.6) when the open string represents $\psi^{1}$ and the closed string represents the tachyon.

Adding (C.4), (C.7), (C.15), (C.19) and (C.25), and carrying out the $\beta$ integrals, we get the total $\mathrm{C}-\mathrm{C}-\mathrm{O}$ amplitude to be

$$
2 i\left(\omega_{1}+\omega_{2}\right) g_{s} \sinh \left(\pi\left|\omega_{1}\right|\right) \sinh \left(\pi\left|\omega_{2}\right|\right)\left\{1-\frac{2}{\pi} \tilde{\lambda}-2 \omega_{1} \omega_{2} \ln \widetilde{\lambda}^{2}\right\} .
$$

This concludes our derivation of (6.31) which was used to find the field redefinition that relates the open string zero mode $\phi$ to the collective coordinate $\widetilde{\phi}$.

We end this appendix by noting that we can also use the procedure described in section 2.4 to evaluate the integral given in (C.1). For example, the integrand of (C.1) may be expressed as:

$$
-d x \wedge d y \frac{i}{\pi}\left[\frac{\omega_{1}}{x^{2}+1}+\frac{\omega_{2} y}{x^{2}+y^{2}}\right] 4 g_{s} \sinh \left(\pi\left|\omega_{1}\right|\right) \sinh \left(\pi\left|\omega_{2}\right|\right)\left[\frac{1}{2} y^{-2}\left(1+2 \omega_{1} \omega_{2} y\right)\right]=d \mathcal{B},
$$

where,

$$
\mathcal{B}=-\frac{i}{\pi} d y\left[\omega_{1} \tan ^{-1} x+\omega_{2} \tan ^{-1} \frac{x}{y}\right] 4 g_{s} \sinh \left(\pi\left|\omega_{1}\right|\right) \sinh \left(\pi\left|\omega_{2}\right|\right)\left[\frac{1}{2} y^{-2}\left(1+2 \omega_{1} \omega_{2} y\right)\right] .
$$

Therefore, (C.1) may be represented as a boundary integral of $\mathcal{B}$ in figure 5 , with the boundaries including the segments $x=\infty, y=1, x=-\infty$, and the regularized boundaries at $y=0$, consisting of the segments $q_{1}=\delta_{1}$ in figure $4(\mathrm{a})$ and (b) and $q_{2}=\delta_{2}$ in figure 4(a) and (c). String field theory instructs us to drop all terms proportional to $\delta_{1}^{-1}, \delta_{2}^{-1}, \ln \delta_{1}$ and $\ln \delta_{2}$ in the final expression. We have checked that this gives the same result as (C.26).

\section{Computation related to gauge parameter redefinition}

As described in section 6.4, in order to find the relation between the string field theory gauge transformation parameter accompanying the state $|0\rangle$, and the rigid $\mathrm{U}(1)$ gauge transformation parameter, we need to compute the disk amplitude $A(\omega)$ of the closed string tachyon vertex operator of energy $\omega$, and the open string vertex operators $I, c \partial c \partial^{2} c / 2$ and I. Furthermore, the open string vertex operators carry Chan-Paton factors so that only one cyclic ordering contributes. In this appendix we shall compute $A(\omega)$.

We shall use the upper half plane representation of the disk with the closed string tachyon vertex operator $c \bar{c} V_{T}$ inserted at $i$ and the vertex operators $I, c \partial c \partial^{2} c / 2$ and $I$ inserted at three points $z_{1}, z_{2}$ and $z_{3}$ respectively on the real axis. The upper half plane 
with three punctures on the boundary and one puncture in the bulk has two real moduli. Let us denote them by $\vec{\beta}=\left(\beta_{1}, \beta_{2}\right)$. Then we have $z_{1}=f_{1}(\vec{\beta}), z_{2}=f_{2}(\vec{\beta})$ and $z_{3}=f_{3}(\vec{\beta})$ where $f_{1}, f_{2}, f_{3}$ are three functions of these moduli. As in (B.3), we describe the choice of local coordinates $w_{a}$ at the punctures via the relations:

$$
z=F_{a}\left(w_{a}, \vec{\beta}\right)=f_{a}(\vec{\beta})+g_{a}(\vec{\beta}) w_{a}+\frac{1}{2} h_{a}(\beta) w_{a}^{2}+\mathcal{O}\left(w_{a}^{3}\right)
$$

We can now use the generalization of (B.2) to express the C-O-O-O amplitude $A(\omega)$ as:

$$
\begin{aligned}
A(\omega)=K_{3} \int d \beta_{1} \wedge d \beta_{2}\left\langle\left\{\sum_{a=1}^{3} \oint_{a} \frac{\partial F_{a}}{\partial \beta_{1}} b(z) d z\right\}\left\{\sum_{a=1}^{3} \oint_{a} \frac{\partial F_{a}}{\partial \beta_{2}} b(z) d z\right\}\right. \\
\left.\times\left\{c \partial c \partial^{2} c\left(z_{2}\right) / 2\right\} c \bar{c} V_{T}(i)\right\rangle
\end{aligned}
$$

where $K_{3}$ is a normalization constant and $\oint_{a}$ is the anti-clockwise contour surrounding $z_{a}$. In writing (D.2) we have made use of the fact that all the vertex operators are dimension zero primary operators, so that conformal transformations by the $F_{a}$ 's leave them unchanged. Using (D.1), (B.6), and evaluating the contour integrals using the $b$-c operator product expansion, we can express (D.2) as:

$$
\begin{aligned}
A(\omega)=\frac{1}{2} K_{2} K_{3} \int & {\left[\left(1+f_{2}^{2}\right) g_{2}^{-3} d h_{2} \wedge d g_{2}+2 f_{2} h_{2} g_{2}^{-3} d g_{2} \wedge d f_{2}\right.} \\
& \left.-2 f_{2} g_{2}^{-2} d h_{2} \wedge d f_{2}+2 g_{2}^{-1} d g_{2} \wedge d f_{2}\right] \\
= & K_{2} K_{3} \int d \mathcal{J},
\end{aligned}
$$

where

$$
\mathcal{J} \equiv-f_{2} g_{2}^{-1} d g_{2}-\frac{1}{2}\left(1+f_{2}^{2}\right) h_{2} g_{2}^{-3} d g_{2}+\frac{1}{2}\left(1+f_{2}^{2}\right) g_{2}^{-2} d h_{2}+d f_{2} .
$$

In this form $\mathcal{J}$ is manifestly invariant under the $\mathrm{SL}(2, R)$ transformation $z \rightarrow(z-c) /(1+c z)$ that keeps the point at $i$ fixed. This can be seen by examining the transformation laws of $f_{i}, g_{i}$ and $h_{i}$ from (D.1) and then substituting them into (D.4). This in turn ensures that when we evaluate the integral in (D.3) by expressing it as a boundary integral of $\mathcal{J}$, we do not need to worry about possible boundary contributions from regions where some open string puncture approaches infinity, since $\infty$ can be brought to a finite point on the real axis using the $\mathrm{SL}(2, R)$ transformation $z \rightarrow(z-c) /(1+c z)$.

Next we shall discuss how to fix the orientation of the integral, i.e. given an integral of the form $\int_{R} d \beta_{1} \wedge d \beta_{2}$ over some finite region $R$, how to decide whether it gives positive or negative result. If we were using the same coordinate system everywhere in the moduli space then this would not be necessary, since an overall sign can always be absorbed inside $K_{3}$. However we shall use different coordinate systems in different regions, and relative 


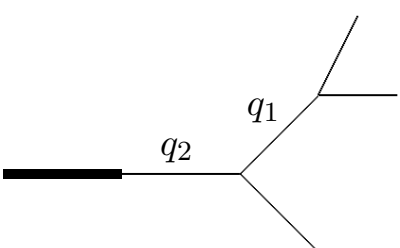

(a)

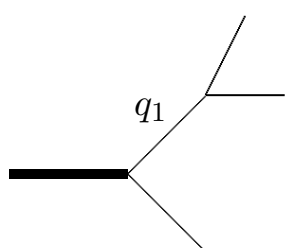

(b)

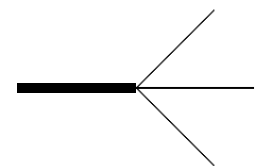

(c)

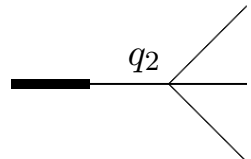

(d)

Figure 10. This figure shows four Feynman diagrams contributing to the disk amplitude with one external closed string and three external open strings. We have not displayed diagrams that differ from each other by permutation of external states. As usual, $q_{1}$ and $q_{2}$ represent the sewing parameters of the corresponding open string propagators.

signs will be important. ${ }^{9}$ For this we shall first make an $\operatorname{SL}(2, R)$ transformation

$$
z^{\prime}=\frac{z-f_{3}}{1+z f_{3}}
$$

to bring the third puncture to 0 , keeping the closed string puncture fixed at $i$. This gives:

$$
z_{1}^{\prime}=\frac{f_{1}-f_{3}}{1+f_{1} f_{3}}, \quad z_{2}^{\prime}=\frac{f_{2}-f_{3}}{1+f_{2} f_{3}}
$$

and

$$
\begin{aligned}
d z_{1}^{\prime} \wedge d z_{2}^{\prime}= & \left(1+f_{1} f_{3}\right)^{-2}\left(1+f_{2} f_{3}\right)^{-2}\left(1+f_{3}^{2}\right) \\
& \times\left[\left(1+f_{3}^{2}\right) d f_{1} \wedge d f_{2}+\left(1+f_{1}^{2}\right) d f_{2} \wedge d f_{3}+\left(1+f_{2}^{2}\right) d f_{3} \wedge d f_{1}\right] .
\end{aligned}
$$

Since $d z_{1}^{\prime} \wedge d z_{2}^{\prime}$ fixes the global orientation of the moduli space of a disk with one closed string puncture and three open string punctures, we need to ensure that the orientations we use in different segments coming from different Feynman diagrams agree with this orientation. We shall take $d z_{1}^{\prime} \wedge d z_{2}^{\prime}$ to be the positive integration measure, i.e. assume that $\int_{R} d z_{1}^{\prime} \wedge d z_{2}^{\prime}$ for some finite region $R$ gives positive result. The other convention where $\int_{R} d z_{2}^{\prime} \wedge d z_{1}^{\prime}$ gives positive result is related to the one used here by a change in the sign of $K_{3}$ introduced in (D.2). Once we decide that $\int_{R} d z_{1}^{\prime} \wedge d z_{2}^{\prime}$ gives positive result, (D.7) can be used to fix the orientation of the moduli space integration coming from different Feynman diagrams. This will be illustrated below.

We shall now analyze the contribution to $A(\omega)$ by expressing this as a sum of four Feynman diagrams shown in figure 10. First we shall argue that the contributions from figure 10(a) and 10(b) vanish. To see this, note that since we are constructing the interaction terms of the Wilsonian effective action in which we remove all the $L_{0}=0$ states from the internal propagators, and since the contributions from internal states with $L_{0}>0$ are suppressed in the limit of large $\alpha$ and/or $\lambda$, we only need to focus on the contributions

\footnotetext{
${ }^{9}$ If we were evaluating the amplitude in string field theory from first principles, then the orientation would have been automatically fixed. Here we are combining string field theory insights with world-sheet methods, and therefore we need to make extra effort to fix the orientation.
} 
where both internal states are tachyons. Now, using the fact that the external open strings are described by vertex operators $I, c \partial c \partial^{2} c / 2$ and $I$, it is easy to see that ghost number conservation prevents the internal open string that connects to the pair of external open strings in figure 10(a) or figure 10(b) to be tachyonic. ${ }^{10}$ For example, if the external open string states are $I$ and $c \partial c \partial^{2} c / 2$, then the lowest $L_{0}$ state that has a three point function with this pair of states is $I$, whereas if the external open string states are $I$ and $I$, then the lowest $L_{0}$ state that has a three point function with this pair of states is $c \partial c \partial^{2} c / 2$. All of these are $L_{0}=0$ states and are prevented from propagating in the internal lines.

Next we shall analyze the contribution from figure 10(d). Let us suppose that the local coordinates at the four punctures at the four point open string interaction vertex are given by $w_{i}=G_{i}(\hat{z}, \tau)$, where $\hat{z}$ is the coordinate on the upper half plane representing this interaction vertex, $\tau$ is the modular parameter of a plane with four boundary punctures and $G_{i}$ 's are some given functions. We can find the range of $\tau$ and the constraints on $G_{i}$ by requiring that the four point interaction vertex correctly interpolates between the $\mathrm{s}, \mathrm{t}$ and $\mathrm{u}$ channel diagrams constructed using the O-O-O vertex given in (4.6), but we shall not need this for our analysis. The only information on $G_{i}(\tau)$ that we shall use is that while it depends on the parameter $\alpha$ that appears in the O-O-O interaction vertex (4.6), it does not depend on the parameter $\lambda$ that appears in the C-O interaction vertex (4.3). Denoting by $z$ the upper half plane coordinate associated with the $\mathrm{C}-\mathrm{O}$ interaction vertex, and using (4.3), we get the sewing relation associated with figure 10(d) to be:

$$
\lambda z G_{4}(\hat{z}, \tau)=-q_{2}, \quad 0 \leq q_{2} \leq 1
$$

where we have assumed that the sewing takes place between the external open string of the C-O vertex and the 4-th external open string of the O-O-O-O vertex. We can rewrite this as

$$
G_{i}(\hat{z}, \tau)=-u / z, \quad u \equiv q_{2} / \lambda, \quad 0 \leq u \leq \lambda^{-1}
$$

Note now that $z$ and $u$ appear in this equation in the combination $z / u$. As a result, the locations of the other three open string punctures in the $z$ plane must have the form $u \phi_{i}(\tau)$ for some functions $\phi_{i}(\tau)$. Furthermore, the local coordinates $w_{a}$ at the open string punctures must be functions of $z / u$ and $\tau$. Therefore we have the expansion:

$$
z=u \phi_{a}(\tau)+u \gamma_{a}(\tau) w_{a}+\frac{u}{2} \eta_{a}(\tau) w_{a}^{2}+\mathcal{O}\left(w_{a}^{3}\right), \quad 1 \leq a \leq 3
$$

Comparing this with (D.1) we see that we have

$$
f_{a}=u \phi_{a}(\tau), \quad g_{a}=u \gamma_{a}(\tau), \quad h_{a}(\tau)=u \eta_{a}(\tau)
$$

\footnotetext{
${ }^{10}$ This can be seen more explicitly by expressing the integral in (D.3) as integral over the variables $q_{i}$ associated to the propagators in figure 10 (a) and (b) and the internal variable $\beta$ of the C-O-O vertex for figure 10(a), and then showing that there are no $q_{1}^{-2}$ singularity in the integrands where $q_{1}$ is the sewing parameter associated with the open string propagator connected to the pair of external open strings. However we shall not describe this analysis here.
} 
Substituting this into the first expression in (D.3) we get the form of the integral inside the region covered by figure $10(\mathrm{~d})$ to be:

$$
I_{(d)}=\frac{1}{2} K_{2} K_{3} \int d u \wedge d \tau\left[u^{-2}\left\{\gamma_{2}^{-3} \eta_{2} \gamma_{2}^{\prime}-\gamma_{2}^{-2} \eta_{2}^{\prime}\right\}+\mathcal{O}\left(u^{0}\right)\right]
$$

where ' denotes derivative with respect to $\tau$.

Let us define $\varepsilon$ such that it can take values 1 or -1 and that $\varepsilon d u \wedge d \tau$ gives the integration measure with the correct sign, i.e. $\varepsilon \int d u \wedge d \tau$ gives positive result when integrated over a finite region in $u$ - $\tau$ space. $\varepsilon$ can be determined from (D.7), but we shall not need this information. We now use the replacement rule (2.11) to replace $\int_{0}^{1 / \lambda} d u u^{-2}$ by $-\lambda$. Terms in the integrand with non-negative powers of $u$ can be made zero by taking the large $\lambda$ limit. This gives the contribution to $A(\omega)$ from figure $10(\mathrm{~d})$ to be

$$
I_{(d)}=-\frac{1}{2} K_{2} K_{3} \lambda \varepsilon \int_{\mathcal{B}_{2}} d \tau\left[\gamma_{2}^{-3} \eta_{2} \gamma_{2}^{\prime}-\gamma_{2}^{-2} \eta_{2}^{\prime}\right]
$$

where $\mathcal{B}_{2}$ is the boundary at $u=\lambda$, with its normal along the direction of increasing $u$.

The contribution from figure 10(c) may be analyzed by using the fact that the integrand in (D.3) is a total derivative and therefore the integral may be expressed as boundary integrals. Now the region of the moduli space covered by figure 10(c) has two boundaries: $\mathcal{B}_{1}$ where the moduli space connects to the $q_{1}=1$ boundary of the region covered by figure $10(\mathrm{~b})$, and $\mathcal{B}_{2}$ where the moduli space connects to the $q_{2}=1$ boundary of the region covered by figure $10(d)$. Therefore we have

$$
I_{(c)}=I_{(c), 1}+I_{(c), 2},
$$

where

$$
I_{(c), i}=K_{2} K_{3} \int_{\mathcal{B}_{i}}\left\{-f_{2} g_{2}^{-1} d g_{2}-\frac{1}{2}\left(1+f_{2}^{2}\right) h_{2} g_{2}^{-3} d g_{2}+\frac{1}{2}\left(1+f_{2}^{2}\right) g_{2}^{-2} d h_{2}+d f_{2}\right\} .
$$

First let us evaluate the contribution from the boundary $\mathcal{B}_{2}$. For this we can use the $u, \tau$ coordinate system introduced in (D.9), and, describing the boundary as the curve $u=1 / \lambda$, express the boundary integral as:

$$
I_{(c), 2}=-K_{2} K_{3} \lambda \varepsilon \int_{\mathcal{B}_{2}} d \tau\left[-\frac{1}{2} \eta_{2} \gamma_{2}^{-3} \gamma_{2}^{\prime}+\frac{1}{2} \gamma_{2}^{-2} \eta_{2}^{\prime}\right]
$$

where we have dropped terms with negative powers of $\lambda$. An extra negative sign in (D.16) comes from the fact that $u=1 / \lambda$ is the lower limit of integration in the region covered by the diagram 10(c). Adding this to (D.13) we get,

$$
I_{(d)}+I_{(c), 2}=0 .
$$

It remains to evaluate the contribution from the boundary $\mathcal{B}_{1}$. Since this boundary is shared with the boundary of the region associated with figure 10(b), we can use the moduli space parameters inherited from this diagram and describe the boundary to be at $q_{1}=1$, 
where $q_{1}$ is the sewing parameter associated with the internal open string propagator of this diagram. ${ }^{11}$ Therefore we shall now study the moduli space parameters inherited from figure 10(b). Let $z$ denote the upper half plane coordinate associated with the C-O-O vertex and $\hat{z}$ denote the upper half plane coordinate associated with the O-O-O vertex. Let us first sew the puncture at $z=\beta$ from the $\mathrm{C}-\mathrm{O}-\mathrm{O}$ vertex with the puncture at $\hat{z}=0$ from the O-O-O vertex, and call the puncture at $z=-\beta$ as puncture 1 , the puncture at $\hat{z}=1$ as puncture 2 and the puncture at $\hat{z}=\infty$ as puncture 3 . In this case the sewing relation takes the form

$$
w_{1} \hat{w}_{1}=-q_{1},
$$

where $w_{1}$ is the local coordinate at the puncture at $\beta$ for the $\mathrm{C}-\mathrm{O}-\mathrm{O}$ vertex, and $\hat{w}_{1}$ is the local coordinate at the puncture at 0 for the O-O-O vertex. Using (4.6) and (4.12), (D.18) may be written as:

$$
\alpha \widetilde{\lambda} \frac{4 \widetilde{\lambda}^{2}+1}{4 \widetilde{\lambda}^{2}} \frac{z-\beta}{(1+\beta z)+\widetilde{\lambda} f(\beta)(z-\beta)} \alpha \frac{2 \hat{z}}{2-\hat{z}}=-q_{1} .
$$

The open string punctures at $z=-\beta, \hat{z}=1$ and $\hat{z}=\infty$ are located in the $z$ plane at

$$
\begin{aligned}
& z_{1}=-\beta, \quad z_{2}=\beta-u \frac{1+\beta^{2}}{1+u(\beta+\widetilde{\lambda} f)}=\frac{\beta+u(\beta \widetilde{\lambda} f-1)}{1+u(\beta+\widetilde{\lambda} f)} \\
& z_{3}=\beta+u \frac{1+\beta^{2}}{1-u(\beta+\widetilde{\lambda} f)}=\frac{\beta-u(\beta \widetilde{\lambda} f-1)}{1-u(\beta+\widetilde{\lambda} f)}
\end{aligned}
$$

where

$$
u \equiv \frac{q_{1}}{2 \alpha^{2} \widetilde{\lambda}} \frac{4 \widetilde{\lambda}^{2}}{4 \widetilde{\lambda}^{2}+1}, \quad 0 \leq u \leq u_{0}, \quad u_{0} \equiv \frac{1}{2 \alpha^{2} \widetilde{\lambda}} \frac{4 \widetilde{\lambda}^{2}}{4 \widetilde{\lambda}^{2}+1}
$$

Furthermore, the local coordinates at the open string punctures are given by,

$$
\begin{aligned}
& w_{1}=\alpha \widetilde{\lambda} \frac{4 \widetilde{\lambda}^{2}+1}{4 \widetilde{\lambda}^{2}} \frac{z+\beta}{(1-\beta z)-\widetilde{\lambda} f(\beta)(z+\beta)}, \\
& w_{2}=-2 \alpha \frac{1-\hat{z}}{1+\hat{z}}=2 \alpha \frac{(z-\beta)\{1+u(\beta+\widetilde{\lambda} f)\}+u\left(1+\beta^{2}\right)}{3 u\left(1+\beta^{2}\right)-(z-\beta)+3 u(\widetilde{\lambda} f+\beta)(z-\beta)}, \\
& w_{3}=\alpha \frac{2}{1-2 \hat{z}}=2 \alpha \frac{(z-\beta)\{1-u(\beta+\widetilde{\lambda} f)\}-u\left(1+\beta^{2}\right)}{3 u\left(1+\beta^{2}\right)+(z-\beta)+3 u(\widetilde{\lambda} f+\beta)(z-\beta)} .
\end{aligned}
$$

\footnotetext{
${ }^{11}$ Actually, $\mathcal{B}_{1}$ consists of six components. A factor of three comes from the possibility of cyclically permuting the external open strings. Another factor of two comes from the freedom of inserting the external open string that attaches to the $\mathrm{C}-\mathrm{O}-\mathrm{O}$ vertex to be either at $\beta$ or at $-\beta$ in the convention of section 4.3 .
} 
Inverting these relations, we get:

$$
\begin{aligned}
z \equiv F_{1}\left(w_{1}, \beta\right)= & -\beta+\frac{4 \widetilde{\lambda}^{2}}{4 \widetilde{\lambda}^{2}+1} \frac{1+\beta^{2}}{\alpha \widetilde{\lambda}} w_{1}-\left(\frac{4 \widetilde{\lambda}^{2}}{4 \widetilde{\lambda}^{2}+1} \frac{1}{\alpha \widetilde{\lambda}}\right)^{2}\left(1+\beta^{2}\right)(\beta+\widetilde{\lambda} f) w_{1}^{2}+\mathcal{O}\left(w_{1}^{3}\right), \\
z \equiv F_{2}\left(w_{2}, \beta\right)= & \frac{\beta+u(\beta \widetilde{\lambda} f-1)}{1+u(\beta+\widetilde{\lambda} f)}+\frac{2 u}{\alpha} \frac{\left(1+\beta^{2}\right)}{\{1+u(\beta+\widetilde{\lambda} f)\}^{2}} w_{2} \\
& -\frac{u}{\alpha^{2}} \frac{\left(1+\beta^{2}\right)\{1-3 u(\beta+\widetilde{\lambda} f)\}}{\{1+u(\beta+\widetilde{\lambda} f)\}^{3}} w_{2}^{2}+\mathcal{O}\left(w_{2}^{3}\right), \\
z \equiv F_{3}\left(w_{3}, \beta\right)= & \frac{\beta-u(\beta \widetilde{\lambda} f-1)}{1-u(\beta+\widetilde{\lambda} f)}+\frac{2 u}{\alpha} \frac{\left(1+\beta^{2}\right)}{\{1-u(\beta+\widetilde{\lambda} f)\}^{2}} w_{3} \\
& +\frac{u}{\alpha^{2}} \frac{\left(1+\beta^{2}\right)\{1+3 u(\beta+\widetilde{\lambda} f)\}}{\{1-u(\beta+\widetilde{\lambda} f)\}^{3}} w_{3}^{2}+\mathcal{O}\left(w_{3}^{3}\right) .
\end{aligned}
$$

Comparing this with the expansion given in (D.1) we get:

$$
\begin{aligned}
& f_{1}=-\beta, \quad f_{2}=\beta-u\left(1+\beta^{2}\right)+u^{2}\left(1+\beta^{2}\right)(\beta+\widetilde{\lambda} f)+\mathcal{O}\left(u^{3}\right), \\
& f_{3}=\beta+u\left(1+\beta^{2}\right)+u^{2}\left(1+\beta^{2}\right)(\beta+\widetilde{\lambda} f)+\mathcal{O}\left(u^{3}\right), \\
& g_{1}=\frac{4 \widetilde{\lambda}^{2}}{4 \widetilde{\lambda}^{2}+1} \frac{1+\beta^{2}}{\alpha \widetilde{\lambda}}, \quad g_{2}=2 \alpha^{-1}\left(1+\beta^{2}\right) u\left\{1-2 u(\beta+\widetilde{\lambda} f)+\mathcal{O}\left(u^{2}\right)\right\}, \\
& g_{3}=2 \alpha^{-1}\left(1+\beta^{2}\right) u\left\{1+2 u(\beta+\widetilde{\lambda} f)+\mathcal{O}\left(u^{2}\right)\right\} \\
& h_{1}=-2\left(\frac{4 \widetilde{\lambda}^{2}}{4 \widetilde{\lambda}^{2}+1} \frac{1}{\alpha \widetilde{\lambda}}\right)^{2}\left(1+\beta^{2}\right)(\beta+\widetilde{\lambda} f), \\
& h_{2}=-2 \frac{u}{\alpha^{2}}\left(1+\beta^{2}\right)\left\{1-6 u(\beta+\widetilde{\lambda} f)+\mathcal{O}\left(u^{2}\right)\right\} \\
& h_{3}=2 \frac{u}{\alpha^{2}}\left(1+\beta^{2}\right)\left\{1+6 u(\beta+\widetilde{\lambda} f)+\mathcal{O}\left(u^{2}\right)\right\} .
\end{aligned}
$$

There are two other configurations related to the one described above by cyclic permutation of $1,2,3$. Since (D.15) involves only $f_{2}, g_{2}$ and $h_{2}$ and fixing the orientation via (D.7) involves also $f_{1}$ and $f_{3}$, we shall quote only these values:

$$
\begin{aligned}
& f_{1}=\beta+u\left(1+\beta^{2}\right)+u^{2}\left(1+\beta^{2}\right)(\beta+\widetilde{\lambda} f)+\mathcal{O}\left(u^{3}\right), \quad f_{2}=-\beta, \quad g_{2}=\frac{1}{\alpha \widetilde{\lambda}} \frac{4 \widetilde{\lambda}^{2}}{4 \widetilde{\lambda}^{2}+1}\left(1+\beta^{2}\right), \\
& h_{2}=-2\left(\frac{4 \widetilde{\lambda}^{2}}{4 \widetilde{\lambda}^{2}+1} \frac{1}{\alpha \widetilde{\lambda}}\right)^{2}\left(1+\beta^{2}\right)(\beta+\widetilde{\lambda} f), \\
& f_{3}=\beta-u\left(1+\beta^{2}\right)+u^{2}\left(1+\beta^{2}\right)(\beta+\widetilde{\lambda} f)+\mathcal{O}\left(u^{3}\right),
\end{aligned}
$$

and

$$
\begin{aligned}
f_{1} & =\beta-u\left(1+\beta^{2}\right)+u^{2}\left(1+\beta^{2}\right)(\beta+\widetilde{\lambda} f)+\mathcal{O}\left(u^{3}\right), \\
f_{2} & =\beta+u\left(1+\beta^{2}\right)+u^{2}\left(1+\beta^{2}\right)(\beta+\widetilde{\lambda} f)+\mathcal{O}\left(u^{3}\right), \\
g_{2} & =2 \alpha^{-1}\left(1+\beta^{2}\right) u\left\{1+2 u(\beta+\widetilde{\lambda} f)+\mathcal{O}\left(u^{2}\right)\right\}, \\
h_{2} & =2 \frac{u}{\alpha^{2}}\left(1+\beta^{2}\right)\left\{1+6 u(\beta+\widetilde{\lambda} f)+\mathcal{O}\left(u^{2}\right)\right\}, \\
f_{3} & =-\beta .
\end{aligned}
$$


We shall now fix the orientation of the integral. For all three cases, the integration measure (D.7) takes the form, for small $u$,

$$
d z_{1}^{\prime} \wedge d z_{2}^{\prime}=F(\beta) d \beta \wedge d u
$$

where $F(\beta)$ is a positive function of $\beta$. Therefore, $d \beta \wedge d u$ is the positive integration measure.

We can now evaluate the contribution to $I_{(c) 1}$ given in (D.15) from (D.24), (D.25) and (D.26), by identifying the boundary $\mathcal{B}_{1}$ as the subspace $u=u_{0},(2 \widetilde{\lambda})^{-1} \leq \beta \leq 1$ in each case. The results are given, respectively, by:

$$
\begin{aligned}
& K_{2} K_{3} \int_{(2 \widetilde{\lambda})^{-1}}^{1} d \beta\left[\frac{2}{1+\beta^{2}}+\tilde{\lambda} f^{\prime}(\beta)\right], \\
& K_{2} K_{3} \int_{(2 \widetilde{\lambda})^{-1}}^{1} d \beta\left[-\frac{2}{1+\beta^{2}}-\tilde{\lambda} f^{\prime}(\beta)\right],
\end{aligned}
$$

and

$$
K_{2} K_{3} \int_{(2 \widetilde{\lambda})^{-1}}^{1} d \beta\left[\frac{2}{1+\beta^{2}}+\widetilde{\lambda} f^{\prime}(\beta)\right]
$$

In arriving at the above equations, two minus signs have cancelled. First we see from (D.27) that $-d u \wedge d \beta$ is the positive integration measure. Second, the outward normal to the boundary of the region associated with figure $10(\mathrm{c})$ at $u=u_{0}$ is directed towards the direction of decreasing $u$.

We also need to consider the case where we sew the puncture at $z=-\beta$ from the $\mathrm{C}-\mathrm{O}-\mathrm{O}$ vertex with the puncture at $\hat{z}=0$ from the O-O-O vertex. ${ }^{12}$ Since the C-O-O vertex is symmetric under $\beta \rightarrow-\beta$, the results in this case can be read out from those in the previous case by making a $\beta \rightarrow-\beta, f \rightarrow-f$ change in the results. This would produce an extra minus sign in (D.27) and (D.28)-(D.30) which compensate each other. Therefore the results are identical to those given in (D.28)-(D.30). The sum of all the terms gives:

$$
A(\omega)=2 K_{2} K_{3} \int_{(2 \widetilde{\lambda})^{-1}}^{1} d \beta\left[\frac{2}{1+\beta^{2}}+\widetilde{\lambda} f^{\prime}(\beta)\right]=2 K_{2} K_{3}\left[\frac{\pi}{2}-\frac{\tilde{\lambda}}{2}\right]
$$

where we have used $f\left((2 \widetilde{\lambda})^{-1}\right)=1 / 2+\mathcal{O}\left(\tilde{\lambda}^{-2}\right)$ and $f(1)=0$, and ignored terms containing inverse power of $\tilde{\lambda}$. Using (6.52) we now see that this gives an additional contribution to the closed string tachyon one point function of the from:

$$
-K_{0}^{-1} A(\omega)=-2 K_{2} K_{3} K_{0}^{-1}\left[\frac{\pi}{2}-\frac{\tilde{\lambda}}{2}\right] .
$$

We shall now determine the ratio $K_{2} K_{3} / K_{0}$. For this consider a C-O-O-O amplitude where all external open string states are tachyons, described by the vertex operator $c$, but they carry the same Chan-Paton factors as the original vertex operators so that only

\footnotetext{
${ }^{12}$ Alternatively, as discussed near the end of section 4.3, we could simply extend the range of $\beta$ in (D.28)(D.30) all the way to $\beta=2 \widetilde{\lambda}$. This gives the same result after we use the result $f(1 / \beta)=f(-\beta)=-f(\beta)$.
} 
one cyclic ordering contributes... Since $c$ is a primary operator of dimension -1 , we have $F_{a} \circ c(0)=\left(F_{a}^{\prime}\left(w_{a}=0\right)\right)^{-1} c\left(z_{a}\right)=g_{a}^{-1} c\left(z_{a}\right)$. In that case, starting from the analog of (D.2):

$$
\begin{aligned}
& \widetilde{A}(\omega)=K_{3} \int d \beta_{1} \wedge d \beta_{2}\langle\left\{\sum_{a} \oint_{a} \frac{\partial F_{a}}{\partial \beta_{1}} b(z) d z\right\}\left\{\sum_{a} \oint_{a} \frac{\partial F_{a}}{\partial \beta_{2}} b(z) d z\right\} \\
&\left.\times\left(g_{1} g_{2} g_{3}\right)^{-1} c\left(z_{1}\right) c\left(z_{2}\right) c\left(z_{3}\right) c \bar{c} V_{T}(i)\right\rangle,
\end{aligned}
$$

and following the same steps as outlined below (D.2), we arrive at the analog of (D.3):

$$
\widetilde{A}(\omega)=K_{2} K_{3} \int\left(g_{1} g_{2} g_{3}\right)^{-1}\left[\left(1+f_{3}^{2}\right) d f_{2} \wedge d f_{1}+\left(1+f_{1}^{2}\right) d f_{3} \wedge d f_{2}+\left(1+f_{2}^{2}\right) d f_{1} \wedge d f_{3}\right] .
$$

For figure 10(b), we can use (D.24) to express this as:

$$
\widetilde{A}_{(b)}(\omega)=-K_{2} K_{3} \frac{4 \widetilde{\lambda}^{2}+1}{4 \widetilde{\lambda}^{2}} \int \frac{\alpha^{3} \widetilde{\lambda}}{\left(1+\beta^{2}\right) u^{2}} d \beta \wedge d u
$$

where we have kept the leading term in the expansion in a power series in $u$. Note that this is the contribution from one Feynman diagram and we have not summed over cyclic permutations. Furthermore, using (D.27) we see that $d \beta \wedge d u$ is the positive integration measure. Now using $u=\left\{1+\left(4 \widetilde{\lambda}^{2}\right)^{-1}\right\}^{-1} q /\left(2 \alpha^{2} \widetilde{\lambda}\right)$ and the replacement rule (2.11), we can carry out integration over $q$ and express the leading contribution from figure 10(b) to the amplitude with one closed string tachyon and three open string tachyons to be:

$$
\widetilde{A}_{(b)}(\omega)=K_{2} K_{3}\left(\frac{4 \widetilde{\lambda}^{2}+1}{4 \widetilde{\lambda}^{2}}\right)^{2} 2 \alpha^{2} \widetilde{\lambda} \int_{1 / 2 \widetilde{\lambda}}^{1} d \beta \frac{\alpha^{3} \widetilde{\lambda}}{\left(1+\beta^{2}\right)} .
$$

On the other hand, it follows from figure 10(b) that this contribution should be proportional to the product of a C-O-O vertex with two open string tachyons and a closed string tachyon as external states, an open string tachyon propagator and an O-O-O vertex with three open string tachyons as external states. Now we see from (B.14) that the product of the C-O-O vertex and the open string tachyon propagator is given by:

$$
-2 g_{s} \sinh (\pi|\omega|) \alpha^{2} \widetilde{\lambda}^{2} \frac{1}{\pi} \int_{1 / 2 \widetilde{\lambda}}^{1} d \beta\left(1+\beta^{2}\right)^{-1}\left(1+\frac{1}{4 \widetilde{\lambda}^{2}}\right)^{2} .
$$

On the other hand, the O-O-O vertex, with all external tachyon states, can be computed using (4.6) and (6.45). This is given by

$$
-K_{0} \alpha^{3}
$$

Multiplying (D.37) by (D.38) we get the expected contribution to figure 10(b):

$$
\widetilde{A}_{(b)}(\omega)=2 K_{0} \alpha^{3} g_{s} \sinh (\pi|\omega|) \alpha^{2} \widetilde{\lambda}^{2} \frac{1}{\pi} \int_{1 / 2 \widetilde{\lambda}}^{1} d \beta\left(1+\beta^{2}\right)^{-1}\left(1+\frac{1}{4 \widetilde{\lambda}^{2}}\right)^{2} .
$$


Comparing (D.36) and (D.39), we get

$$
\frac{K_{2} K_{3}}{K_{0}}=\frac{1}{\pi} g_{s} \sinh (\pi|\omega|) .
$$

Substituting this into (D.32), we get,

$$
-K_{0}^{-1} A(\omega)=-\frac{2}{\pi} g_{s} \sinh (\pi|\omega|)\left[\frac{\pi}{2}-\frac{\tilde{\lambda}}{2}\right] .
$$

This concludes the derivation of (6.53).

Finally we would like to note that the integral (D.3), having a total derivative as the integrand, can also be evaluated using the method described in section 2.4. This is given as an integral of $\mathcal{J}$ over the boundaries of the moduli space, corresponding to the segments $q_{1}=\delta_{1}$ in figure 10 (a) and (b) and $q_{2}=\delta_{2}$ in figure 10 (a) and (c). Each of these boundary segments actually represent three separate terms, obtained by cyclic permutation of the three external lines. We have checked that the integral, evaluated this way, gives the same final result (D.31) after dropping all terms proportional to $\delta_{1}^{-1}, \delta_{2}^{-1}, \ln \delta_{1}$ and $\ln \delta_{2}$ in the final expression.

\section{E Closed string tachyon one point function on the annulus with a more general $\mathrm{C}-\mathrm{O}-\mathrm{O}$ vertex}

In the analysis of the C-O-O amplitude in section 4.3, we could include on the right hand side of (4.12) a multiplicative function of $\beta$ that takes value 1 at $\beta=(2 \widetilde{\lambda})^{-1}$, but is otherwise arbitrary. In this appendix we shall show that the final result is independent of the choice of this function. For simplicity we shall assume that this function differs from 1 by infinitesimal amount, so that we can express this as $1+\wp(\beta)$ and work to first order in $\wp(\beta)$. Therefore we replace (4.12) by

$$
w_{a}=(1+\wp(\beta)) \alpha \widetilde{\lambda} \frac{4 \widetilde{\lambda}^{2}+1}{4 \widetilde{\lambda}^{2}} \frac{z-z_{a}}{\left(1+z_{a} z\right)+\widetilde{\lambda} f\left(z_{a}\right)\left(z-z_{a}\right)}, \quad a=1,2, \quad z_{1}=-\beta, \quad z_{2}=\beta .
$$

The analysis may be easily generalized to the case of finite multiplicative function.

We shall first outline the general strategy for dealing with this problem. Let us suppose that we have an amplitude where one of the O legs of the C-O-O vertex gets connected by a propagator to some other vertex. Let $w$ be the local coordinate of the $\mathrm{O}$ of the C-O-O vertex and $w^{\prime}$ be the local coordinate of the other $\mathrm{O}$ to which it gets connected. Then the sewing relation takes the form:

$$
w w^{\prime}=-\widetilde{q}, \quad 0 \leq \widetilde{q} \leq 1,
$$

where we have labelled the sewing parameter by $\widetilde{q}$ to distinguish this from the original sewing parameter $q$ that appeared for $\wp(\beta)=0$. When we express this in terms of the upper half plane coordinates associated with the two vertices, then the $\wp(\beta)$ dependence appears in the form of a multiplicative factor $(1+\wp)$ on the left hand side of (E.2). Therefore 
the sewing relations with the modified vertex may be read out from the original sewing relations by making the identification:

$$
\widetilde{q}=(1+\wp(\beta)) q .
$$

The effect of this can be analyzed from the results of section 3. As shown there, a scaling of this form does not affect integrals of the form $\int d q q^{-2}$ - if we replace $q$ in terms of $\widetilde{q}$ using (E.3) and then use the replacement rule (2.11), we get the same result. However a rescaling of this type does affect integrals of the form $\int d q q^{-1}$. This is because according to (2.11), we are instructed to drop terms of the form $\int_{0}^{1} d q q^{-1}$ from the amplitudes. For the original choice of the $\mathrm{C}-\mathrm{O}-\mathrm{O}$ vertex, we drop such terms in the range $0 \leq q \leq 1$, whereas with the new choice of the C-O-O vertex, we drop such terms in the range $0 \leq \widetilde{q} \leq 1$. Since the latter translates to the range $0 \leq q \leq(1-\wp(\beta))$, we see that effectively with the new choice of the C-O-O vertex, we shall have an extra contribution:

$$
\int_{1-\wp(\beta)}^{1} d q q^{-1}=\wp(\beta) .
$$

This leads to the following simple prescription: identify all diagrams where a C-O-O vertex appears, and if $q$ denotes the sewing parameter of the propagator to which one of the $\mathrm{O}$ legs of the C-O-O vertex connects, then for every $\int_{0}^{1} d q q^{-1}$ in the original expression, we have an extra contribution where we replace $\int_{0}^{1} d q q^{-1}$ by $\wp(\beta)$, leaving the rest of the expression unchanged. If both ends of the propagator connects to the $\mathrm{O}$ legs of the $\mathrm{C}-\mathrm{O}-\mathrm{O}$ vertex, then the contribution is doubled.

Therefore our task is to identify all diagrams in our analysis that contain a C-O-O vertex. We list them below:

1. Contributions to the C-C-O amplitude from figure 4 (b) and (c) involve the C-O-O vertex. This will modify the contributions given in (C.12) and (C.18).

2. Contribution to the annulus one point function given in figure $7(\mathrm{c})$ involves the C-O-O vertex. This, in turn, will modify the contribution given in (6.9).

Besides these, there are two further effects that need to be taken into account:

1. The modification of the $\mathrm{C}-\mathrm{O}-\mathrm{O}$ vertex also affects the $\psi^{1}$ exchange diagrams where the internal $\psi^{1}$ connects to the $\mathrm{O}$ of the $\mathrm{C}-\mathrm{O}-\mathrm{O}$ vertex. This has to be analyzed separately. Figure $9(\mathrm{~b})$ is the only diagram of this type in our analysis. This will modify the analysis of section 6.2 and appendix B.

2. Contribution to the C-O-O-O amplitude shown in figure 10 (b) involves the C-O-O vertex. In this case, besides the effect mentioned above, there will be additional dependence on $\wp(\beta)$ since one of the external vertex operators involve $c \partial c \partial^{2} c / 2$ that can connect to one of the O's of the C-O-O vertex. Since this vertex operator is not of the form $c W$ where $W$ is a dimension one matter primary, the integrand will have a complicated dependence on the local coordinates of the C-O-O vertex, and hence on $\wp(\beta)$. This needs to be taken into account separately. 


\section{E.1 Modification of the annulus one point function}

The off-shell C-O-O vertex appears in figure 7(c). Therefore it modifies the contribution from this diagram as given in (6.9). Since the integration variable $u$ in (6.9) is proportional to the sewing parameter $q$, the additional term is obtained by replacing $\int d u u^{-1}$ term by $2 \wp(\beta)$. The extra factor of 2 comes from the fact that the propagator now connects two O's of the C-O-O vertex. This gives the following additional contribution to $g(\omega)$ :

$$
\frac{2}{\pi} \int_{1 /(2 \widetilde{\lambda})}^{1} d \beta \wp(\beta)\left[\tilde{\lambda} f^{\prime}(\beta)+\frac{1}{4} \frac{1+\beta^{2}}{\beta^{2}}+\frac{2}{1+\beta^{2}} \omega^{2}\right]
$$

\section{E.2 Modification of the C-C-O disk amplitude and its consequences}

Contributions to the C-C-O amplitude from figure 4 (b) and (c) involve the C-O-O vertex, and their expressions, for C's given by a pair of closed string tachyons of energies $\omega_{1}$ and $\omega_{2}$ and $\mathrm{O}$ given by an external open string state with vertex operator $c \partial X$, have been given in (C.12) and (C.18). Since the contribution to (C.18) is related to the one in (C.12) by $\omega_{1} \leftrightarrow \omega_{2}$ exchange, let us focus on (C.12). Here the integration variable $u$ is proportional to $q$, so according to the algorithm given earlier we simply need to replace $\int d u u^{-1}$ by $\wp(\beta)$ to get the extra term. Adding the contribution from (C.18) by $\omega_{1} \leftrightarrow \omega_{2}$ exchange, we get the net extra contribution to be:

$$
\begin{aligned}
& -\frac{2 i}{\pi} g_{s} \sinh \left(\pi\left|\omega_{1}\right|\right) \sinh \left(\pi\left|\omega_{2}\right|\right)\left(\omega_{1}+\omega_{2}\right) \int_{|\xi| \geq 4 \widetilde{\lambda} /\left(4 \widetilde{\lambda}^{2}-1\right)} d \xi \wp(\beta)\left\{\frac{1}{\xi^{2}}+2 \omega_{1} \omega_{2} \frac{1}{1+\xi^{2}}\right\}, \\
& \xi= \pm \frac{2 \beta}{1-\beta^{2}} .
\end{aligned}
$$

Converting the integration variable from $\xi$ to $\beta$, and ignoring terms with inverse powers of $\widetilde{\lambda}$, we get

$$
-\frac{2 i}{\pi} g_{s} \sinh \left(\pi\left|\omega_{1}\right|\right) \sinh \left(\pi\left|\omega_{2}\right|\right)\left(\omega_{1}+\omega_{2}\right) \int_{1 /(2 \widetilde{\lambda})}^{1} d \beta \wp(\beta)\left\{\frac{1+\beta^{2}}{\beta^{2}}+8 \omega_{1} \omega_{2} \frac{1}{1+\beta^{2}}\right\} .
$$

This term needs to be added to (6.33). This in turn requires us to add a new term to the effective action (6.34) and therefore a new term to the required field redefinition (6.37), (6.39). This will lead to an additional contribution to $g(\omega)$ as in (6.43). Since the analysis is identical to that leading from (6.33) to (6.43), we only quote the final result for the additional contribution to $g(\omega)$ :

$$
-\frac{1}{\pi} \int_{1 /(2 \widetilde{\lambda})}^{1} d \beta \wp(\beta)\left\{\frac{1+\beta^{2}}{2 \beta^{2}}+4 \omega^{2} \frac{1}{1+\beta^{2}}\right\} .
$$

\section{E.3 Modification of the $\psi^{1}$ exchange contribution}

We shall now study the effect of modifying the C-O-O vertex on the $\psi^{1}$ exchange contribution shown in figure 9(b) and analyzed in appendix B. In particular our modification of the C-O-O vertex has the effect of replacing $w_{a}$ by $w_{a}(1-\wp(\beta))$ in (B.3). Following the 
subsequent analysis in appendix B, it is easy to see that the net effect of this change is to replace

$$
\frac{1}{g_{a}} \frac{\partial g_{a}}{\partial \beta} \rightarrow \frac{1}{g_{a}} \frac{\partial g_{a}}{\partial \beta}-\wp^{\prime}(\beta),
$$

in (B.7). $h_{a} / g_{a}^{2}$ and $f_{a}$ are not affected by $\wp(\beta)$. This gives a net extra contribution to (B.7) of the form

$$
-4 K_{1} K_{2} \int_{1 /(2 \widetilde{\lambda})}^{1} d \beta \wp^{\prime}(\beta) \tilde{\lambda} f(\beta) .
$$

Multiplying this by the $\psi^{1}$ propagator $K_{0}^{-1} / 2$, we get the net extra contribution to the annulus one point function of the closed string tachyon from the $\psi^{1}$ exchange diagram:

$$
-2 K_{1} K_{2} K_{0}^{-1} \int_{1 /(2 \widetilde{\lambda})}^{1} d \beta \wp^{\prime}(\beta) \widetilde{\lambda} f(\beta)=-2 \frac{g_{s}}{\pi} \sinh (\pi|\omega|) \int_{1 /(2 \widetilde{\lambda})}^{1} d \beta \wp(\beta) \widetilde{\lambda} f^{\prime}(\beta),
$$

where in the second step we have used (B.15) and carried out integration by parts over $\beta$, ignoring the boundary terms since $\wp(\beta) f(\beta)$ vanishes at the boundaries. This translates to the following additional contribution to $g(\omega)$ :

$$
-\frac{1}{\pi} \int_{1 /(2 \widetilde{\lambda})}^{1} d \beta \wp(\beta) \widetilde{\lambda} f^{\prime}(\beta) .
$$

\section{E.4 Modification of the C-O-O-O amplitude and its consequences}

We shall now examine how the modification of the C-O-O vertex affects the results of section 6.4 and appendix D. The C-O-O vertex appears in figure 10(b). Even though we have argued that the contribution from this diagram vanishes, this affects the final result indirectly, since the contribution from the boundary $\mathcal{B}_{1}$ of the region of the moduli space associated with figure 10(c) was evaluated by parametrizing this boundary by the coordinate system inherited from figure 10 (b). In particular the modification of the C-O-O vertex changes (D.19) to

$$
(1+\wp(\beta)) \alpha \widetilde{\lambda} \frac{4 \widetilde{\lambda}^{2}+1}{4 \widetilde{\lambda}^{2}} \frac{z-\beta}{(1+\beta z)+\widetilde{\lambda} f(\beta)(z-\beta)} \alpha \frac{2 \hat{z}}{2-\hat{z}}=-\widetilde{q},
$$

where $\widetilde{q}$ is the new sewing parameter. Comparing this with (D.19) we get the relation:

$$
q=\widetilde{q}(1-\wp(\beta)) .
$$

This has the effect of replacing $u$ by $\widetilde{u}(1-\wp(\beta))$ in (D.20), (D.22), with

$$
\widetilde{u}=\frac{1}{2 \alpha^{2} \widetilde{\lambda}} \frac{4 \widetilde{\lambda}^{2}}{4 \widetilde{\lambda}^{2}+1} \widetilde{q}, \quad 0 \leq \widetilde{u} \leq u_{0}, \quad u_{0} \equiv \frac{1}{2 \alpha^{2} \widetilde{\lambda}} \frac{4 \widetilde{\lambda}^{2}}{4 \widetilde{\lambda}^{2}+1} .
$$

$\widetilde{q}=1$ defines the modified boundary $\mathcal{B}_{1}$. Furthermore the expression for $w_{1}$ in (D.22) is multiplied by a factor of $(1+\wp(\beta))$ since $w_{1}$ is inherited from the local coordinate on the $\mathrm{C}-\mathrm{O}-\mathrm{O}$ vertex. The net effect of these changes may be summarized as follows:

1. In (D.24), $u$ is replaced by $\widetilde{u}(1-\wp(\beta))$, the expression for $g_{1}$ is multiplied by $(1-\wp(\beta))$ and the expression for $h_{1}$ is multiplied by $(1-2 \wp(\beta))$. 
2. In (D.25), $u$ is replaced by $\widetilde{u}(1-\wp(\beta))$, the expression for $g_{2}$ is multiplied by $(1-\wp(\beta))$ and the expression for $h_{2}$ is multiplied by $(1-2 \wp(\beta))$.

3. In (D.26), $u$ is replaced by $\widetilde{u}(1-\wp(\beta))$.

The effect of these changes is to modify (D.28), (D.29) and (D.30) by additive terms of the form:

$$
-K_{2} K_{3} \tilde{\lambda} \int_{1 /(2 \widetilde{\lambda})}^{1} d \beta \wp^{\prime}(\beta) f(\beta), \quad K_{2} K_{3} \tilde{\lambda} \int_{1 /(2 \widetilde{\lambda})}^{1} d \beta \wp^{\prime}(\beta) f(\beta), \quad-K_{2} K_{3} \widetilde{\lambda} \int_{1 /(2 \widetilde{\lambda})}^{1} d \beta \wp^{\prime}(\beta) f(\beta),
$$

respectively. The second set of contributions, obtained by exchanging the punctures at $\beta$ and $-\beta$ of the $\mathrm{C}-\mathrm{O}-\mathrm{O}$ vertex, gives identical contribution. Therefore the net modification of the amplitude $A(\omega)$ is:

$$
-2 K_{2} K_{3} \widetilde{\lambda} \int_{1 /(2 \widetilde{\lambda})}^{1} d \beta \wp^{\prime}(\beta) f(\beta)=2 K_{2} K_{3} \widetilde{\lambda} \int_{1 /(2 \widetilde{\lambda})}^{1} d \beta \wp(\beta) f^{\prime}(\beta)
$$

where we have performed an integration by parts. Since the contribution to the annulus one point function is given by $-2 K_{0}^{-1} A(\omega)$, the modification of the annulus one point function is given by:

$$
-4 K_{2} K_{3} K_{0}^{-1} \widetilde{\lambda} \int_{1 /(2 \widetilde{\lambda})}^{1} d \beta \wp(\beta) f^{\prime}(\beta)=-\frac{2}{\pi} g_{s} \sinh (\pi|\omega|) \tilde{\lambda} \int_{1 /(2 \widetilde{\lambda})}^{1} d \beta \wp(\beta) f^{\prime}(\beta),
$$

where in the second step we have used (D.40). This, in turn, gives an additional contribution to $g(\omega)$ of the form:

$$
-\frac{1}{\pi} \widetilde{\lambda} \int_{1 /(2 \widetilde{\lambda})}^{1} d \beta \wp(\beta) f^{\prime}(\beta) .
$$

We now see that the sum of (E.5), (E.8), (E.12) and (E.19) vanishes. Therefore the modification of the C-O-O vertex has no effect on $g(\omega)$.

Open Access. This article is distributed under the terms of the Creative Commons Attribution License (CC-BY 4.0), which permits any use, distribution and reproduction in any medium, provided the original author(s) and source are credited.

\section{References}

[1] J. Polchinski, Combinatorics of boundaries in string theory, Phys. Rev. D 50 (1994) R6041 [hep-th/9407031] [INSPIRE].

[2] M.B. Green and M. Gutperle, Effects of D instantons, Nucl. Phys. B 498 (1997) 195 [hep-th/9701093] [INSPIRE].

[3] M. Billó, M. Frau, I. Pesando, F. Fucito, A. Lerda and A. Liccardo, Classical gauge instantons from open strings, JHEP 02 (2003) 045 [hep-th/0211250] [INSPIRE].

[4] B. Balthazar, V.A. Rodriguez and X. Yin, $Z Z$ instantons and the non-perturbative dual of $c=1$ string theory, arXiv: 1907.07688 [INSPIRE]. 
[5] S.R. Das and A. Jevicki, String field theory and physical interpretation of D=1 strings, Mod. Phys. Lett. A 5 (1990) 1639 [inSPIRE].

[6] A.M. Sengupta and S.R. Wadia, Excitations and interactions in $d=1$ string theory, Int. J. Mod. Phys. A 6 (1991) 1961 [InSPIRE].

[7] D.J. Gross and I.R. Klebanov, Fermionic string field theory of $c=1$ two-dimensional quantum gravity, Nucl. Phys. B 352 (1991) 671 [InSPIRE].

[8] I.R. Klebanov, String theory in two-dimensions, in Spring school on string theory and quantum gravity (to be followed by workshop), (1991) [hep-th/9108019] [INSPIRE].

[9] G.W. Moore, M.R. Plesser and S. Ramgoolam, Exact $S$ matrix for $2 D$ string theory, Nucl. Phys. B 377 (1992) 143 [hep-th/9111035] [InSPIRE].

[10] B. Balthazar, V.A. Rodriguez and X. Yin, Multi-instanton calculus in $c=1$ string theory, arXiv: 1912.07170 [INSPIRE].

[11] C. de Lacroix, H. Erbin, S.P. Kashyap, A. Sen and M. Verma, Closed superstring field theory and its applications, Int. J. Mod. Phys. A 32 (2017) 1730021 [arXiv:1703.06410] [INSPIRE].

[12] A. Sen, String field theory as world-sheet UV regulator, JHEP 10 (2019) 119 [arXiv: 1902.00263] [INSPIRE].

[13] P.V. Larocca and C. Maccaferri, BCFT and OSFT moduli: an exact perturbative comparison, Eur. Phys. J. C 77 (2017) 806 [arXiv: 1702.06489] [INSPIRE].

[14] A. Sen, Fixing an ambiguity in two dimensional string theory using string field theory, JHEP 03 (2020) 005 [arXiv: 1908. 02782] [INSPIRE].

[15] A. Sen, D-instanton perturbation theory, JHEP 08 (2020) 075 [arXiv:2002.04043] [INSPIRE].

[16] B. Balthazar, V.A. Rodriguez and X. Yin, private communication.

[17] A. Sen, Divergent $\Longrightarrow$ complex amplitudes in two dimensional string theory, JHEP 02 (2021) 086 [arXiv: 2003.12076] [inSPIRE].

[18] H. Hata and B. Zwiebach, Developing the covariant Batalin-Vilkovisky approach to string theory, Annals Phys. 229 (1994) 177 [hep-th/9301097] [INSPIRE].

[19] B. Zwiebach, Quantum open string theory with manifest closed string factorization, Phys. Lett. B 256 (1991) 22 [INSPIRE].

[20] B. Zwiebach, Closed string field theory: quantum action and the B-V master equation, Nucl. Phys. B 390 (1993) 33 [hep-th/9206084] [INSPIRE].

[21] B. Zwiebach, Oriented open-closed string theory revisited, Annals Phys. 267 (1998) 193 [hep-th/9705241] [INSPIRE].

[22] I.A. Batalin and G.A. Vilkovisky, Gauge algebra and quantization, Phys. Lett. B 102 (1981) 27 [INSPIRE].

[23] I.A. Batalin and G.A. Vilkovisky, Quantization of gauge theories with linearly dependent generators, Phys. Rev. D 28 (1983) 2567 [Erratum ibid. 30 (1984) 508] [INSPIRE].

[24] M. Henneaux and C. Teitelboim, Quantization of gauge systems, Princeton University Press, Princeton, NJ, U.S.A. (1992).

[25] A. Sen and B. Zwiebach, A note on gauge transformations in Batalin-Vilkovisky theory, Phys. Lett. B 320 (1994) 29 [hep-th/9309027] [INSPIRE]. 
[26] H. Kajiura, Homotopy algebra morphism and geometry of classical string field theory, Nucl. Phys. B 630 (2002) 361 [hep-th/0112228] [INSPIRE].

[27] H. Kajiura, Noncommutative homotopy algebras associated with open strings, Rev. Math. Phys. 19 (2007) 1 [math.QA/0306332] [INSPIRE].

[28] H. Erbin, C. Maccaferri, M. Schnabl and J. Vošmera, Classical algebraic structures in string theory effective actions, JHEP 11 (2020) 123 [arXiv:2006.16270] [INSPIRE].

[29] T. Erler and H. Matsunaga, Mapping between Witten and Lightcone string field theories, arXiv:2012.09521 [INSPIRE].

[30] A. Sen, Wilsonian effective action of superstring theory, JHEP 01 (2017) 108 [arXiv: 1609.00459] [INSPIRE].

[31] H. Erbin, C. Maccaferri and J. Vošmera, Localization of effective actions in heterotic string field theory, JHEP 02 (2020) 059 [arXiv: 1912.05463] [INSPIRE].

[32] N. Nakanishi, Covariant quantization of the electromagnetic field in the Landau gauge, Prog. Theor. Phys. 35 (1966) 1111 [INSPIRE].

[33] B. Lautrup, Canonical quantum electrodynamics in covariant gauges, Kgl. Dan. Vid. Se. Mat. Fys. Medd. 35 (11) (1967) 1.

[34] E. Witten, Noncommutative geometry and string field theory, Nucl. Phys. B 268 (1986) 253 [INSPIRE].

[35] S.F. Moosavian and R. Pius, Hyperbolic geometry of superstring perturbation theory, arXiv:1703.10563 [INSPIRE].

[36] S.F. Moosavian and R. Pius, Hyperbolic geometry and closed bosonic string field theory. Part I. The string vertices via hyperbolic Riemann surfaces, JHEP 08 (2019) 157 [arXiv: 1706.07366] [INSPIRE].

[37] S.F. Moosavian and R. Pius, Hyperbolic geometry and closed bosonic string field theory. Part II. The rules for evaluating the quantum BV master action, JHEP 08 (2019) 177 [arXiv: 1708.04977] [inSPIRE].

[38] K. Costello and B. Zwiebach, Hyperbolic string vertices, arXiv:1909.00033 [INSPIRE].

[39] M. Cho, Open-closed hyperbolic string vertices, JHEP 05 (2020) 046 [arXiv: 1912.00030] [INSPIRE].

[40] T. Erler, S. Konopka and I. Sachs, One loop tadpole in heterotic string field theory, JHEP 11 (2017) 056 [arXiv : 1704.01210] [InSPIRE].

[41] A. Sen, Cutkosky rules and unitarity (violation) in D-instanton amplitudes, arXiv:2012.00041 [INSPIRE].

[42] D. Ghoshal and A. Sen, Gauge and general coordinate invariance in nonpolynomial closed string theory, Nucl. Phys. B 380 (1992) 103 [hep-th/9110038] [INSPIRE].

[43] E. Witten, Superstring perturbation theory revisited, arXiv:1209.5461 [INSPIRE].

[44] A. Sen, Gauge invariant 1PI effective action for superstring field theory, JHEP 06 (2015) 022 [arXiv: 1411.7478] [INSPIRE].

[45] M.B. Green and P. Vanhove, D instantons, strings and M-theory, Phys. Lett. B 408 (1997) 122 [hep-th/9704145] [INSPIRE]. 
[46] M.B. Green, M. Gutperle and P. Vanhove, One loop in eleven-dimensions, Phys. Lett. B 409 (1997) 177 [hep-th/9706175] [INSPIRE].

[47] E. Kiritsis and B. Pioline, On $R^{4}$ threshold corrections in IIB string theory and $(p, q)$ string instantons, Nucl. Phys. B 508 (1997) 509 [hep-th/9707018] [INSPIRE].

[48] B. Pioline and E. Kiritsis, U duality and D-brane combinatorics, Phys. Lett. B 418 (1998) 61 [hep-th/9710078] [INSPIRE].

[49] N.A. Obers and B. Pioline, Eisenstein series and string thresholds, Commun. Math. Phys. 209 (2000) 275 [hep-th/9903113] [INSPIRE].

[50] M.B. Green, H.-H. Kwon and P. Vanhove, Two loops in eleven-dimensions, Phys. Rev. D 61 (2000) 104010 [hep-th/9910055] [INSPIRE].

[51] M.B. Green and P. Vanhove, Duality and higher derivative terms in M-theory, JHEP 01 (2006) 093 [hep-th/0510027] [INSPIRE].

[52] A. Basu, The $D^{4} R^{4}$ term in type IIB string theory on $T^{2}$ and $U$-duality, Phys. Rev. D 77 (2008) 106003 [arXiv:0708.2950] [INSPIRE].

[53] A. Basu, The $D^{6} R^{4}$ term in type IIB string theory on $T^{2}$ and $U$-duality, Phys. Rev. D 77 (2008) 106004 [arXiv:0712.1252] [INSPIRE].

[54] B. Pioline, $R^{4}$ couplings and automorphic unipotent representations, JHEP 03 (2010) 116 [arXiv: 1001.3647 ] [INSPIRE].

[55] M.B. Green, J.G. Russo and P. Vanhove, Automorphic properties of low energy string amplitudes in various dimensions, Phys. Rev. D 81 (2010) 086008 [arXiv:1001.2535] [INSPIRE].

[56] M.B. Green, S.D. Miller, J.G. Russo and P. Vanhove, Eisenstein series for higher-rank groups and string theory amplitudes, Commun. Num. Theor. Phys. 4 (2010) 551 [arXiv: 1004.0163] [INSPIRE].

[57] M.B. Green, S.D. Miller and P. Vanhove, Small representations, string instantons, and Fourier modes of Eisenstein series, J. Number Theor. 146 (2015) 187 [arXiv:1111.2983] [INSPIRE].

[58] O. Hohm and H. Samtleben, Exceptional form of D = 11 supergravity, Phys. Rev. Lett. 111 (2013) 231601 [arXiv:1308.1673] [INSPIRE].

[59] M.B. Green, S.D. Miller and P. Vanhove, $\mathrm{SL}(2, Z)$-invariance and D-instanton contributions to the $D^{6} R^{4}$ interaction, Commun. Num. Theor. Phys. 09 (2015) 307 [arXiv:1404.2192] [INSPIRE].

[60] B. Pioline, $D^{6} R^{4}$ amplitudes in various dimensions, JHEP 04 (2015) 057 [arXiv: 1502.03377] [INSPIRE].

[61] G. Bossard and A. Kleinschmidt, Loops in exceptional field theory, JHEP 01 (2016) 164 [arXiv: 1510.07859] [INSPIRE].

[62] G. Bossard and A. Kleinschmidt, Cancellation of divergences up to three loops in exceptional field theory, JHEP 03 (2018) 100 [arXiv:1712.02793] [INSPIRE].

[63] T. Takayanagi and N. Toumbas, A matrix model dual of type $0 B$ string theory in two-dimensions, JHEP 07 (2003) 064 [hep-th/0307083] [INSPIRE]. 
[64] M.R. Douglas, I.R. Klebanov, D. Kutasov, J.M. Maldacena, E.J. Martinec and N. Seiberg, A new hat for the $c=1$ matrix model, in From fields to strings: circumnavigating theoretical physics. A conference in tribute to Ian Kogan, (2003), pg. 1758 [hep-th/0307195] [INSPIRE].

[65] M. Mariño, Lectures on non-perturbative effects in large $N$ gauge theories, matrix models and strings, Fortsch. Phys. 62 (2014) 455 [arXiv: 1206.6272] [INSPIRE].

[66] G.V. Dunne and M. Ünsal, What is QFT? Resurgent trans-series, Lefschetz thimbles, and new exact saddles, PoS LATTICE2015 (2016) 010 [arXiv: 1511.05977] [INSPIRE].

[67] I. Aniceto, G. Basar and R. Schiappa, A primer on resurgent transseries and their asymptotics, Phys. Rept. 809 (2019) 1 [arXiv:1802.10441] [INSPIRE]. 